\title{
Development of a convergent enantioselective synthetic route to (-)-myrocin G
}

Martin Tomanik, ${ }^{+, 1}$ Christos Economou, ${ }^{+, 1}$ Madeline C. Frischling, ${ }^{1}$ Mengzhao Xue, ${ }^{1}$ Victoria A. Marks $^{1}$, Brandon Q. Mercado ${ }^{1}$ and Seth B. Herzon ${ }^{*, 1,2}$

${ }^{1}$ Department of Chemistry, Yale University, New Haven, Connecticut 06520, United States.

${ }^{2}$ Department of Pharmacology, Yale School of Medicine, New Haven, Connecticut 06520, United States

J. Org. Chem.

\section{Supporting Information}

\section{$\underline{\text { Table of Contents }}$}

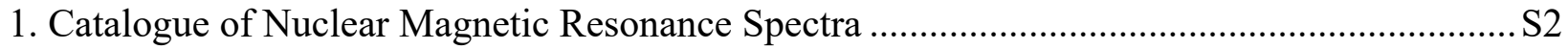

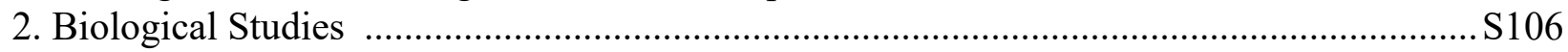

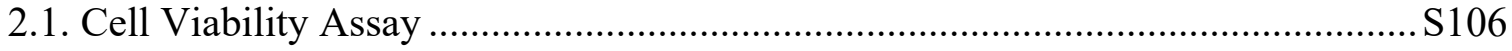

2.2. DNA Cleavage Assay .......................................................................................S107

3.3. DNA Crosslinking Assay .............................................................................. 109

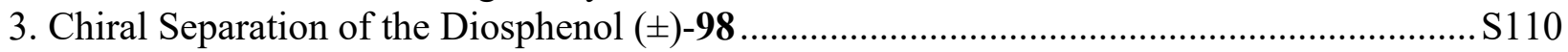

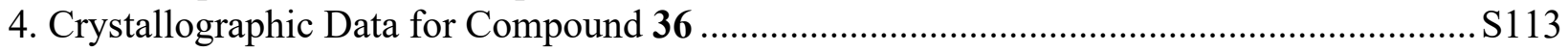

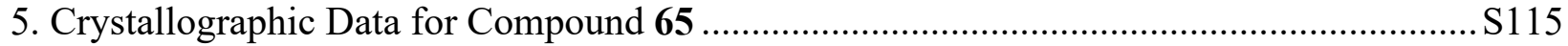

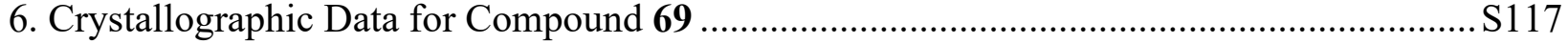

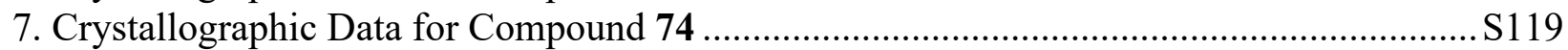

8. Crystallographic Data for Compound 89 ........................................................................ S121

9. Crystallographic Data for Compound (-)-104 ……....................................................... S123 
1. Catalogue of Nuclear Magnetic Resonance Spectra.

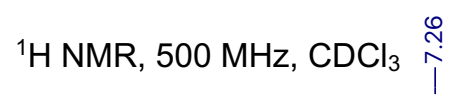
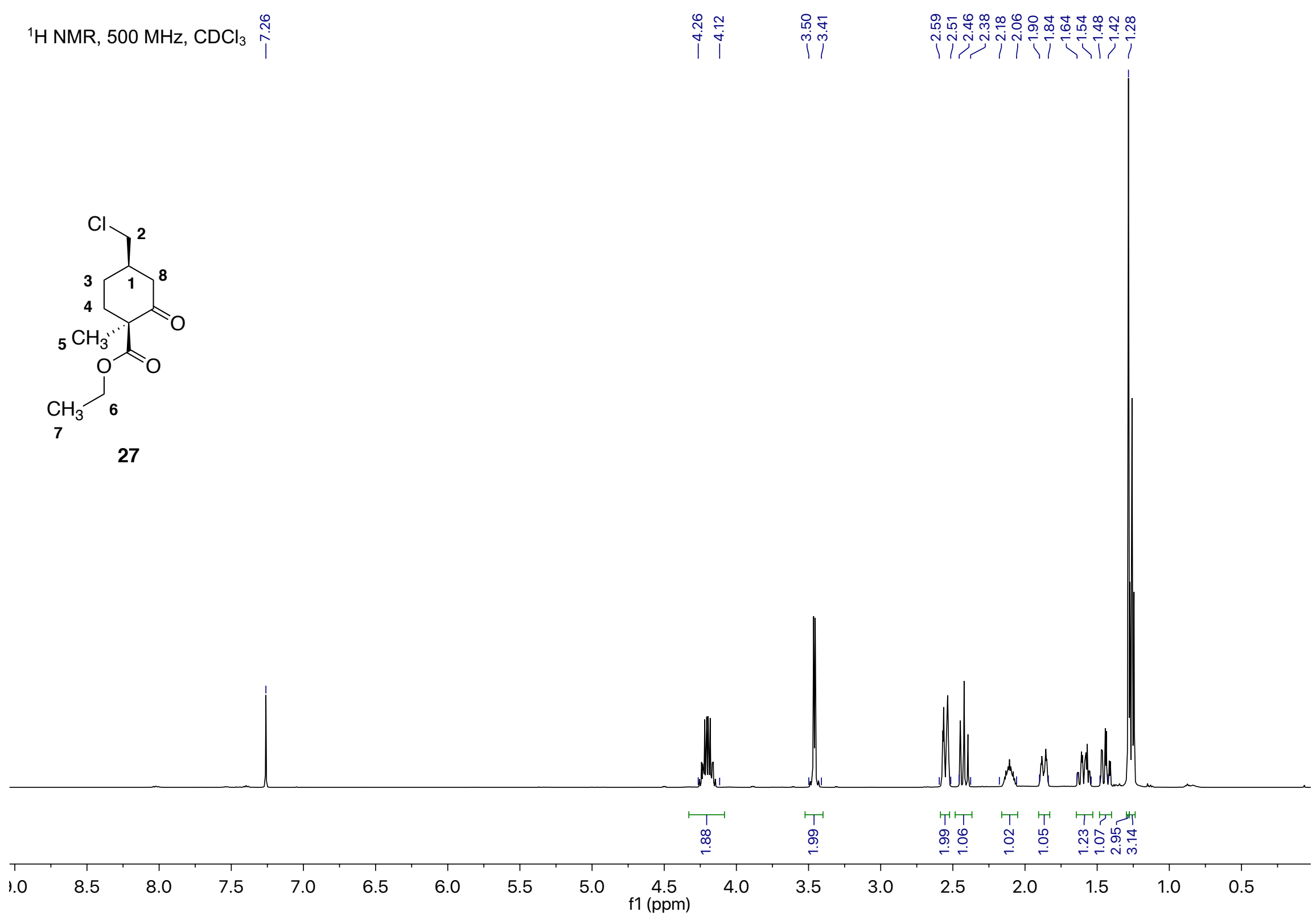
${ }^{13} \mathrm{C} \mathrm{NMR}, 150 \mathrm{MHz}, \mathrm{CDCl}_{3}$

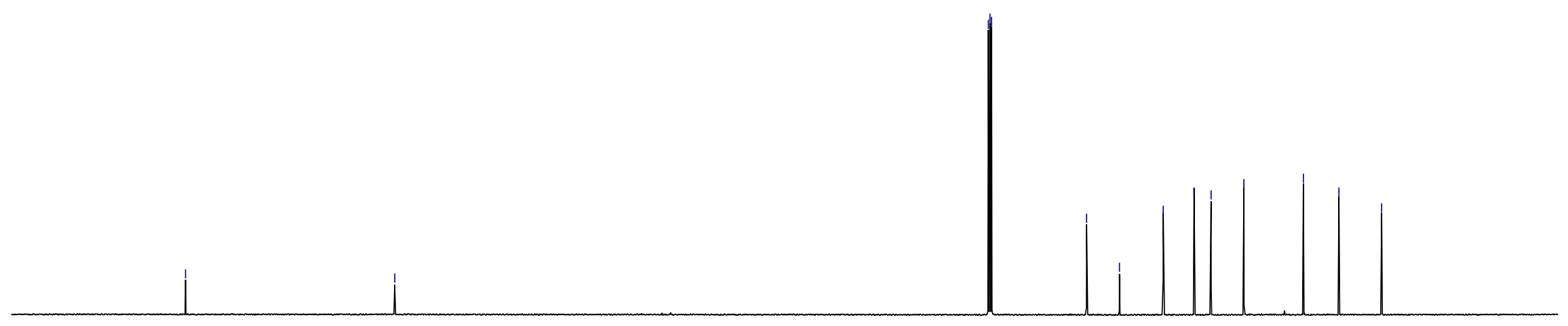

\begin{tabular}{llllllllllllllllllllllllllllll}
\hline 230 & 220 & 210 & 200 & 190 & 180 & 170 & 160 & 150 & 140 & 130 & 120 & 110 & 100 & 90 & 80 & 70 & 60 & 50 & 40 & 30 & 20 & 10 & 0 & -10
\end{tabular} 
${ }^{1} \mathrm{H} \mathrm{NMR}, 500 \mathrm{MHz}, \mathrm{CDCl}_{3}$
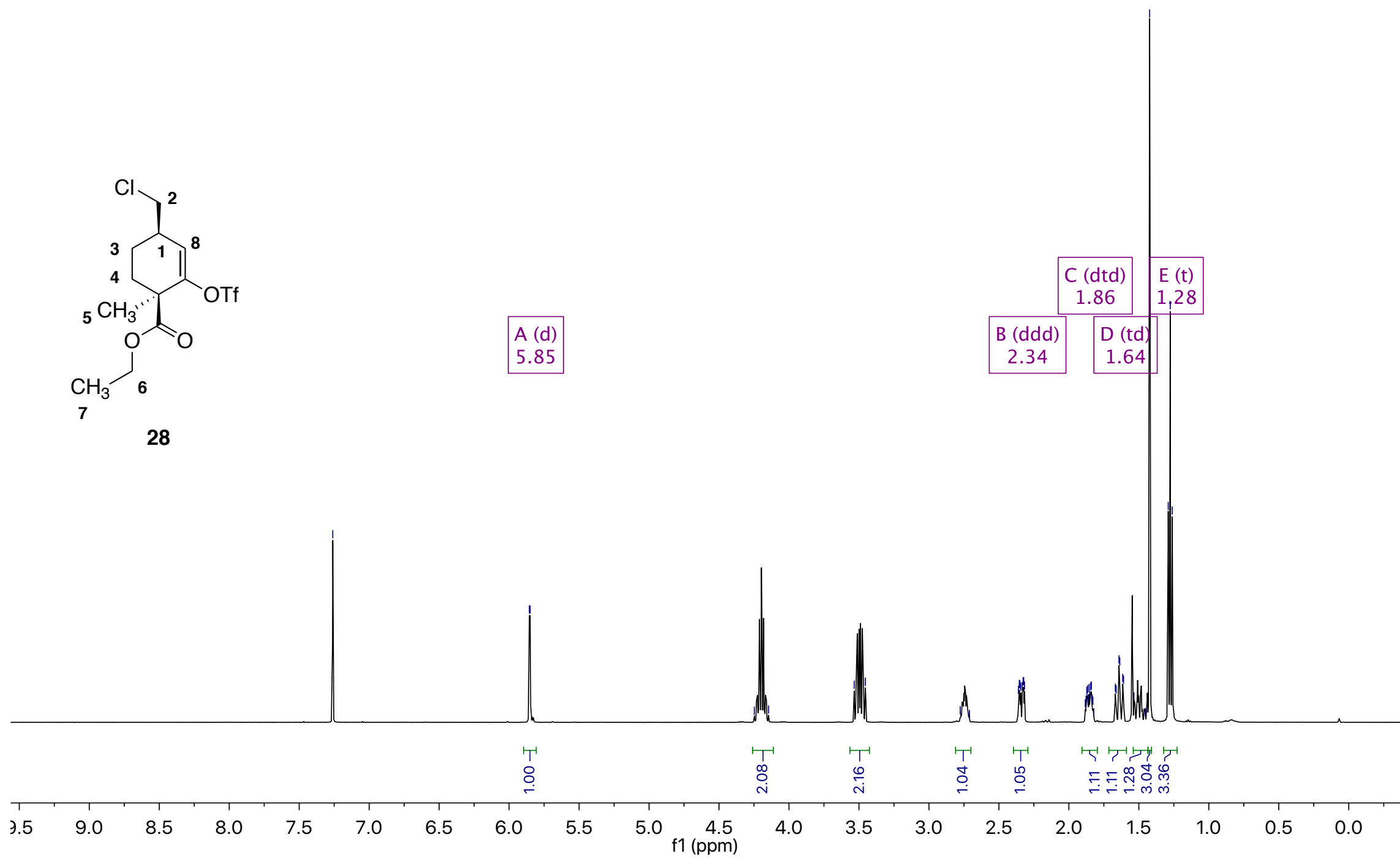
${ }^{13} \mathrm{C}$ NMR, $125 \mathrm{MHz}, \mathrm{CDCl}_{3}$ $\stackrel{\check{m}}{\stackrel{亠}{1}}$

$\stackrel{\circ}{\frac{0}{10}}$ $\stackrel{ }{\circ}$

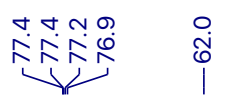

产审

শ্ণ
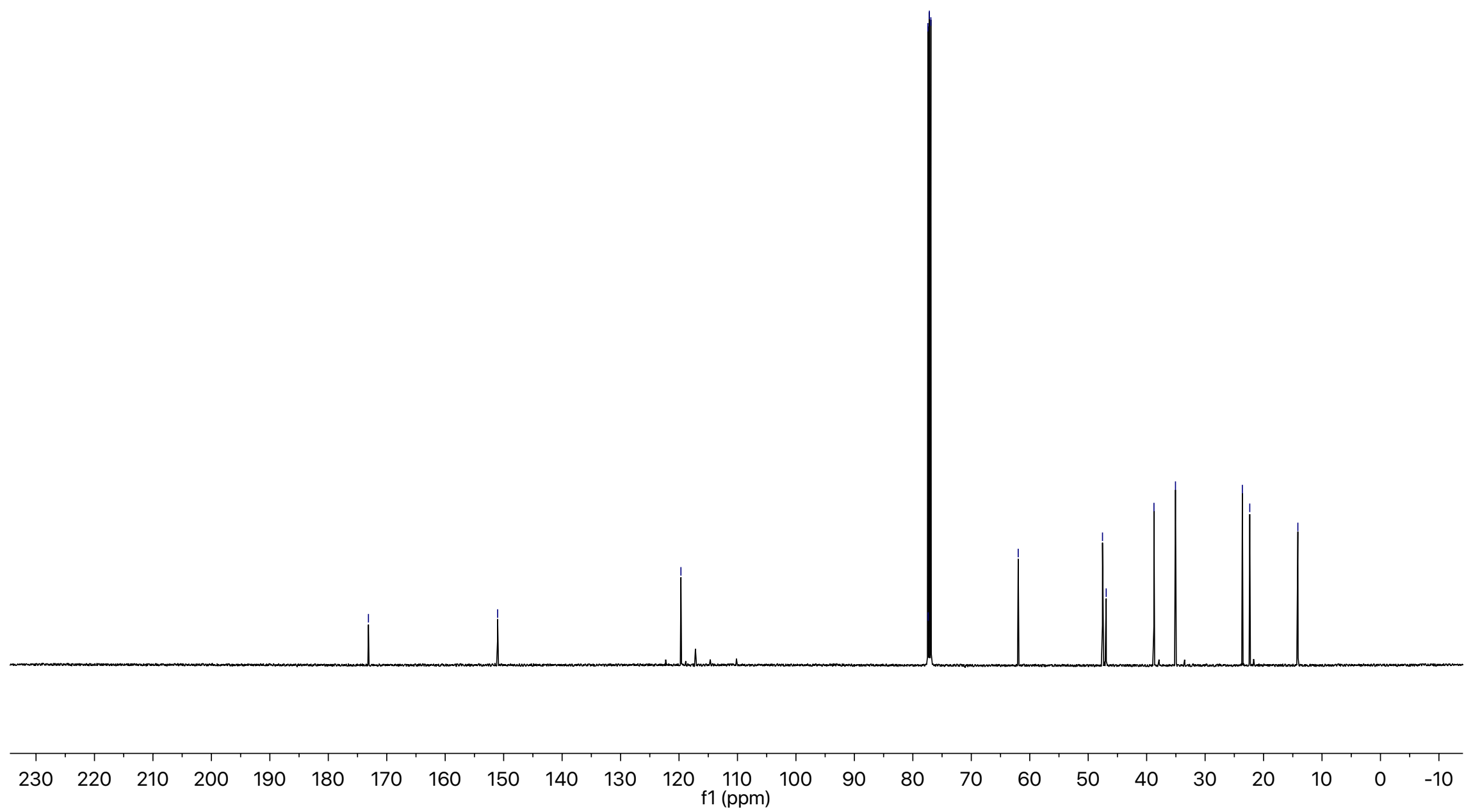

S5 
${ }^{1} \mathrm{H}$ NMR, $500 \mathrm{MHz}, \mathrm{CDCl}_{3}$

䒠

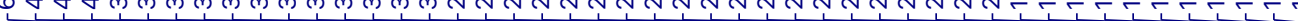

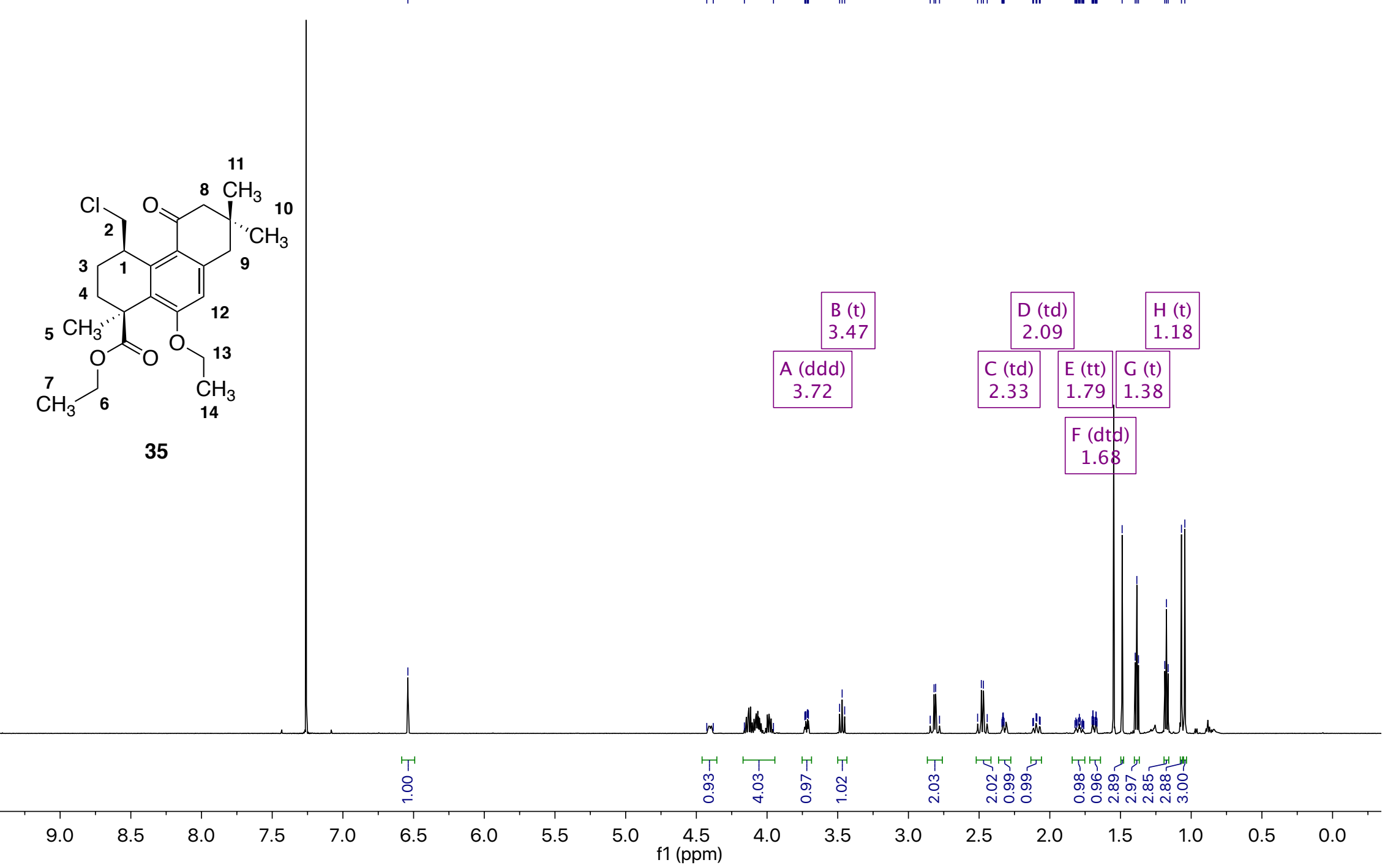


${ }^{13} \mathrm{C} \mathrm{NMR,}, 125 \mathrm{MHz}, \mathrm{CDCl}_{3}$

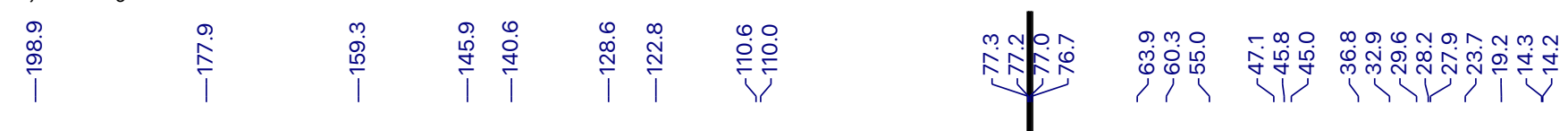


${ }^{1} \mathrm{H} \mathrm{NMR}, 600 \mathrm{MHz}, \mathrm{CDCl}_{3}$

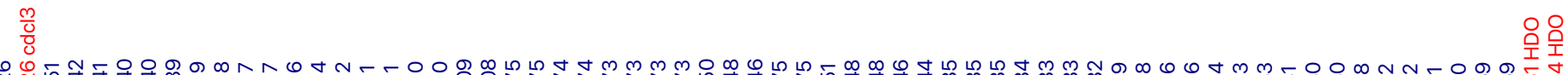

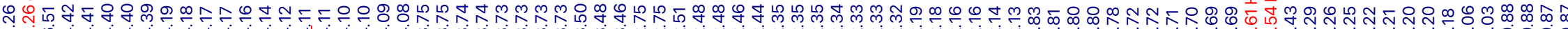

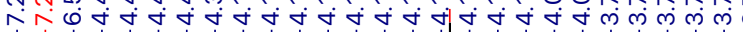

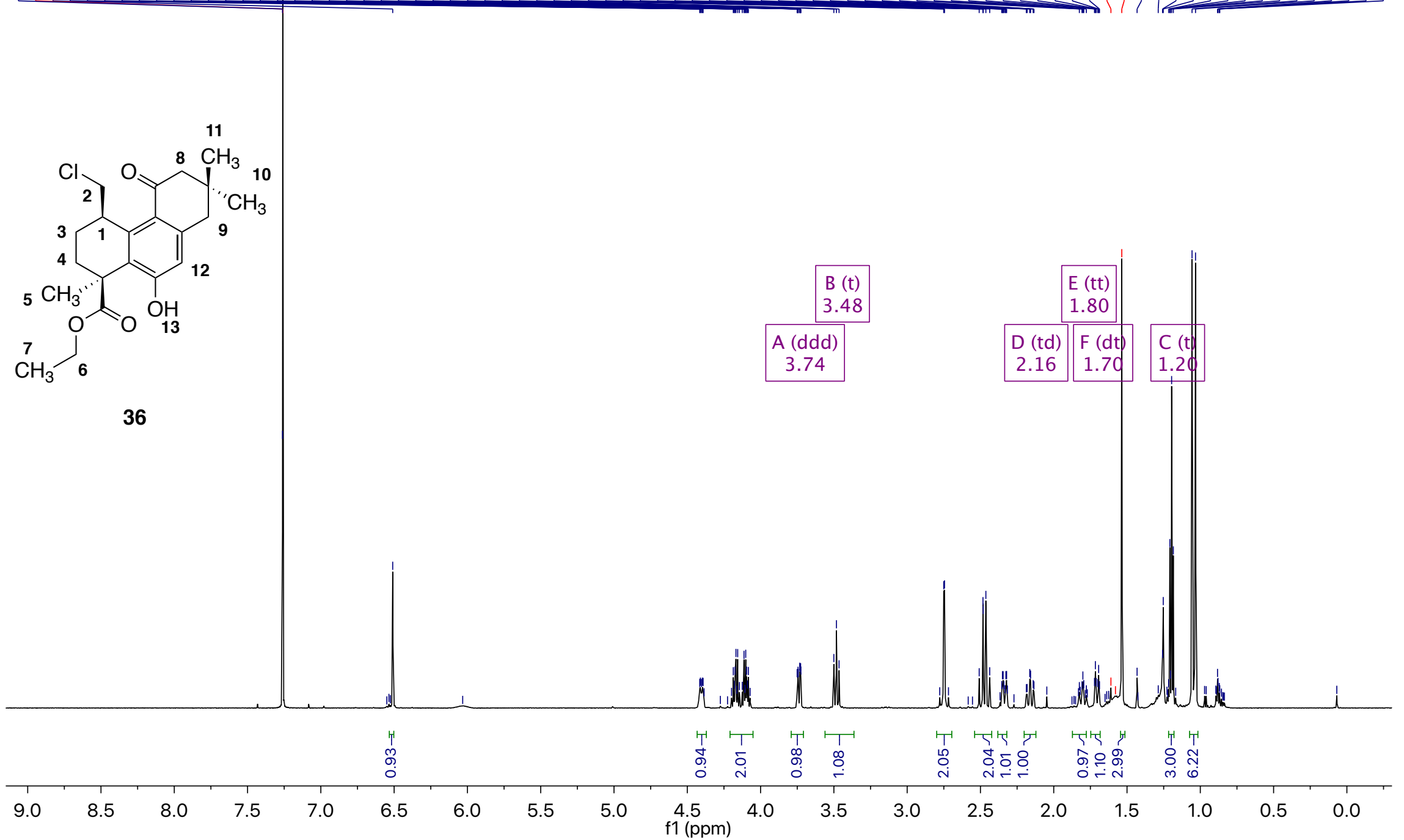


${ }^{1} \mathrm{H} \mathrm{NMR}, 600 \mathrm{MHz}, \mathrm{CDCl}_{3}$

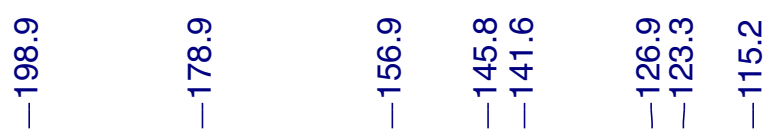

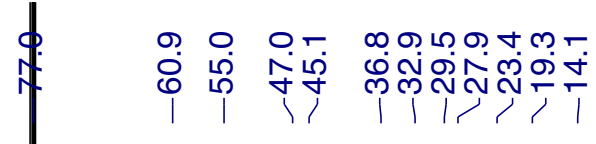

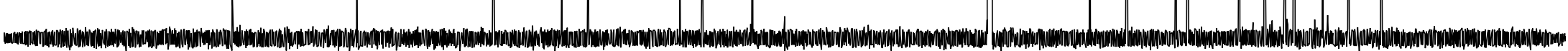

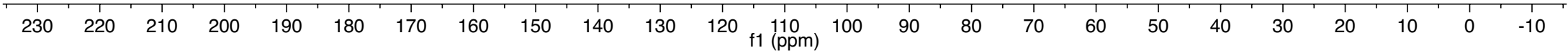


${ }^{1} \mathrm{H} \mathrm{NMR}, 500 \mathrm{MHz}, \mathrm{CDCl}_{3} \quad \stackrel{\stackrel{0}{i}}{i}$
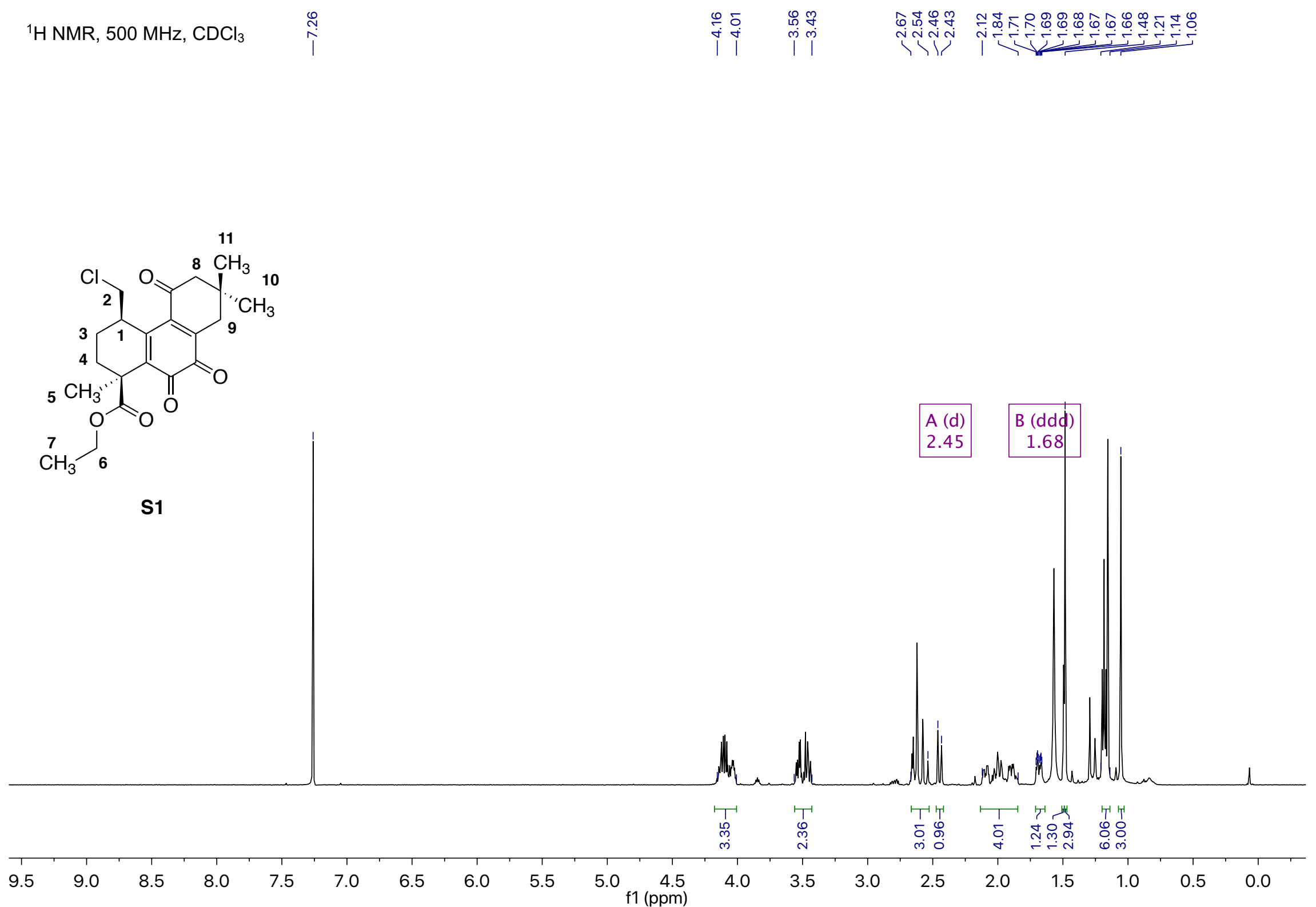
${ }^{13} \mathrm{C}$ NMR, $125 \mathrm{MHz}, \mathrm{CDCl}_{3}$

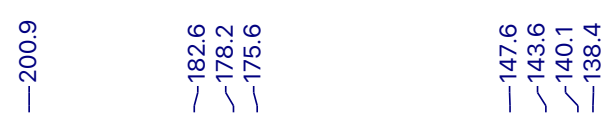

l |
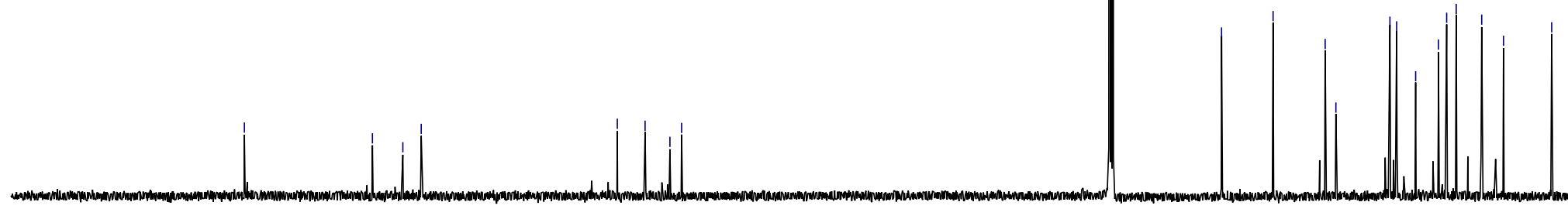

230

$\begin{array}{lllll}220 & 210 & 200 & 190 & 180\end{array}$

$\begin{array}{lllll}170 & 160 & 150 & 140\end{array}$

130

20110100

90

80

70

60

50

$40 \quad 30$

20

10

$0 \quad-10$ 
${ }^{1} \mathrm{H}$ NMR, $500 \mathrm{MHz}, \mathrm{CDCl}_{3}$

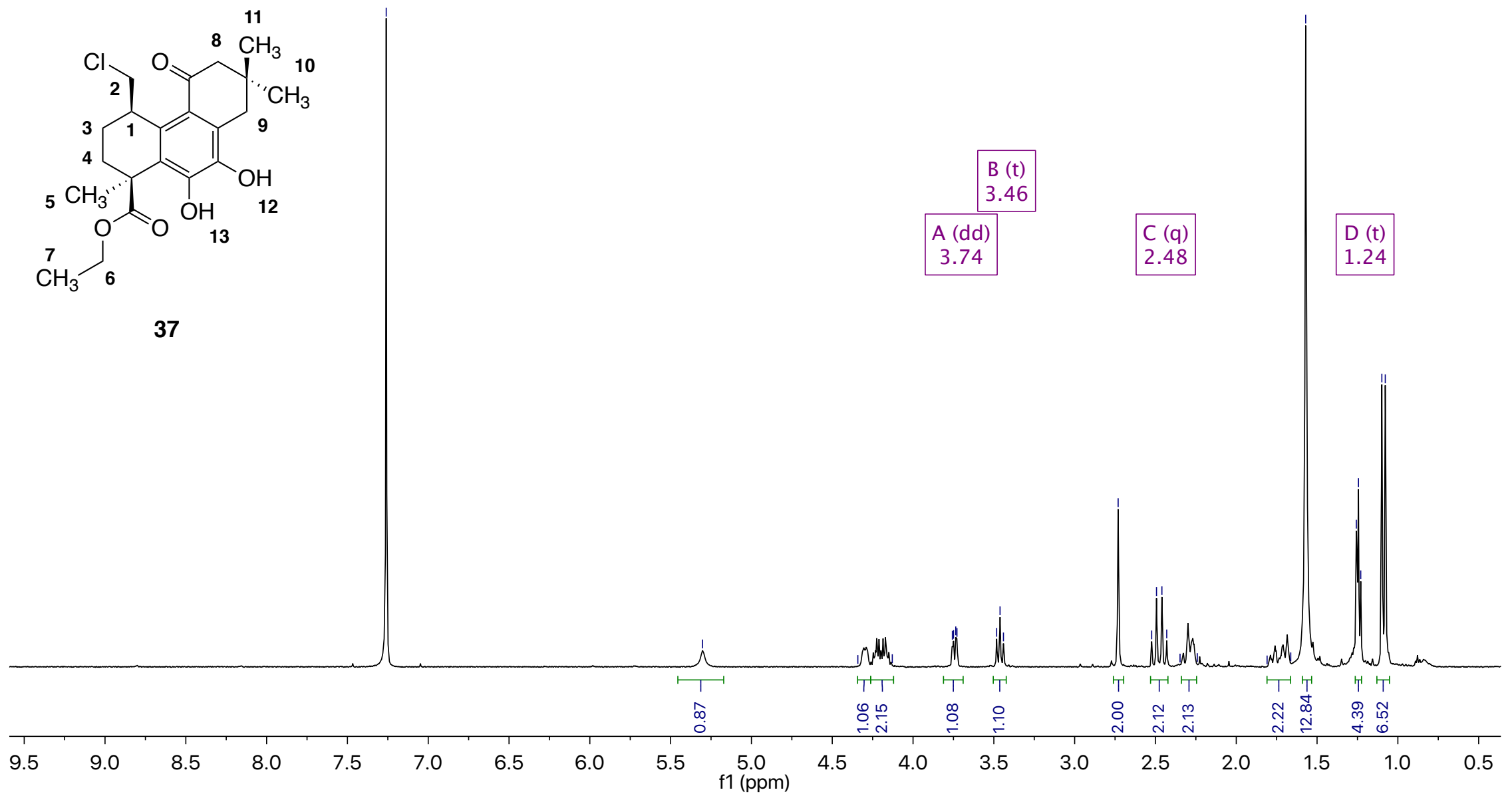


${ }^{13} \mathrm{C}$ NMR, $125 \mathrm{MHz}, \mathrm{CDCl}_{3}$

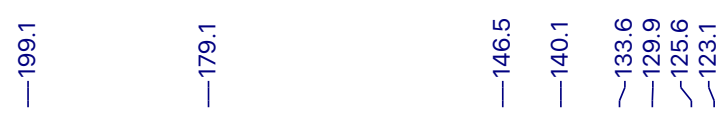

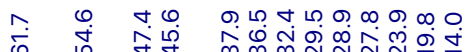

111111

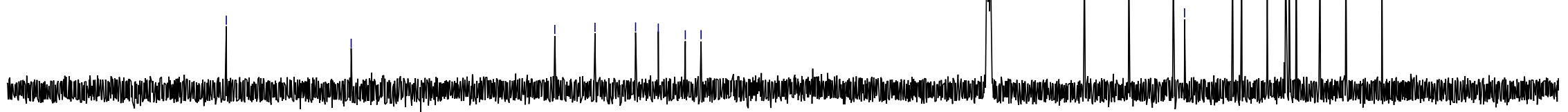


${ }^{1} \mathrm{H} \mathrm{NMR}, 500 \mathrm{MHz}, \mathrm{CDCl}_{3}$

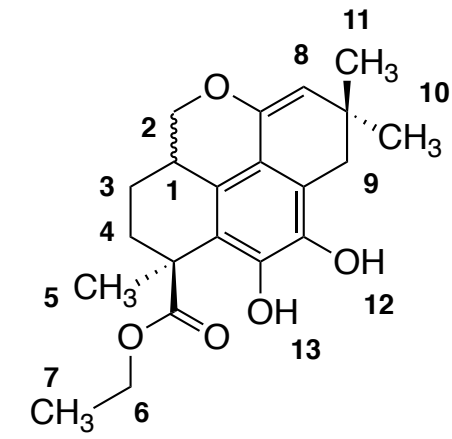

40

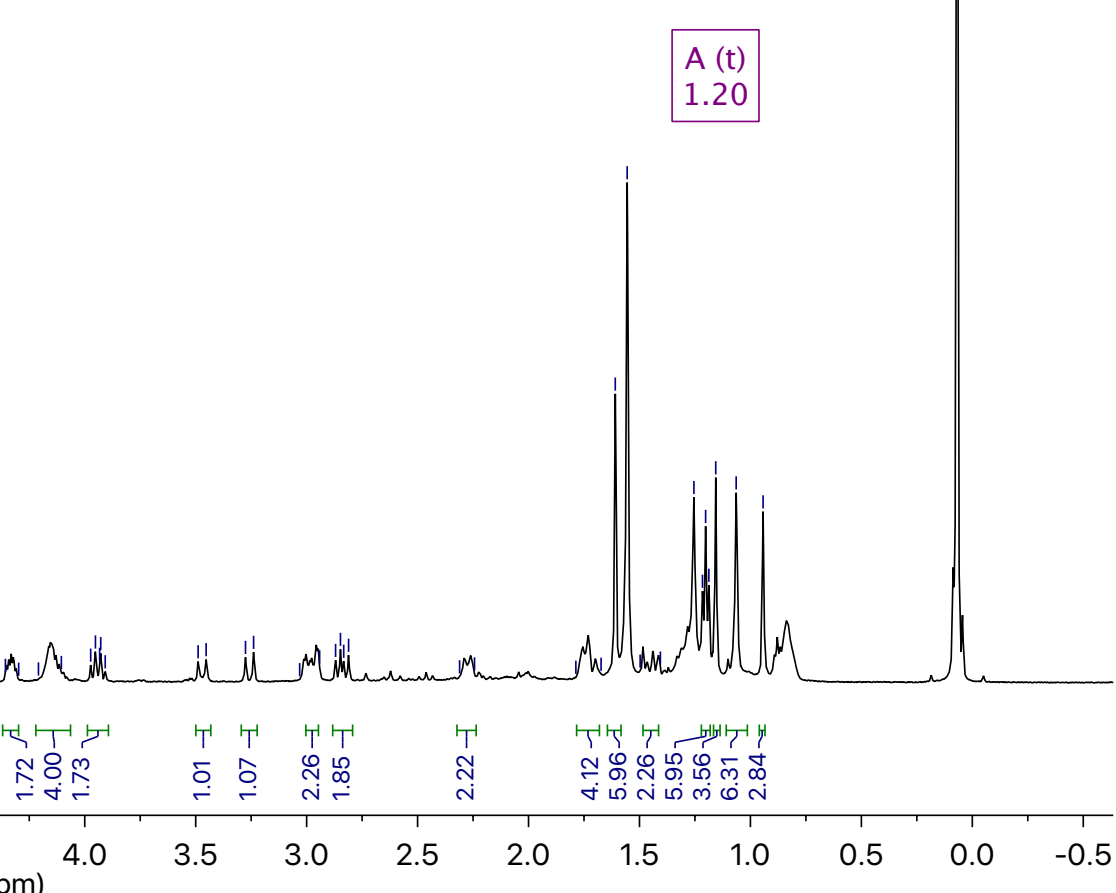




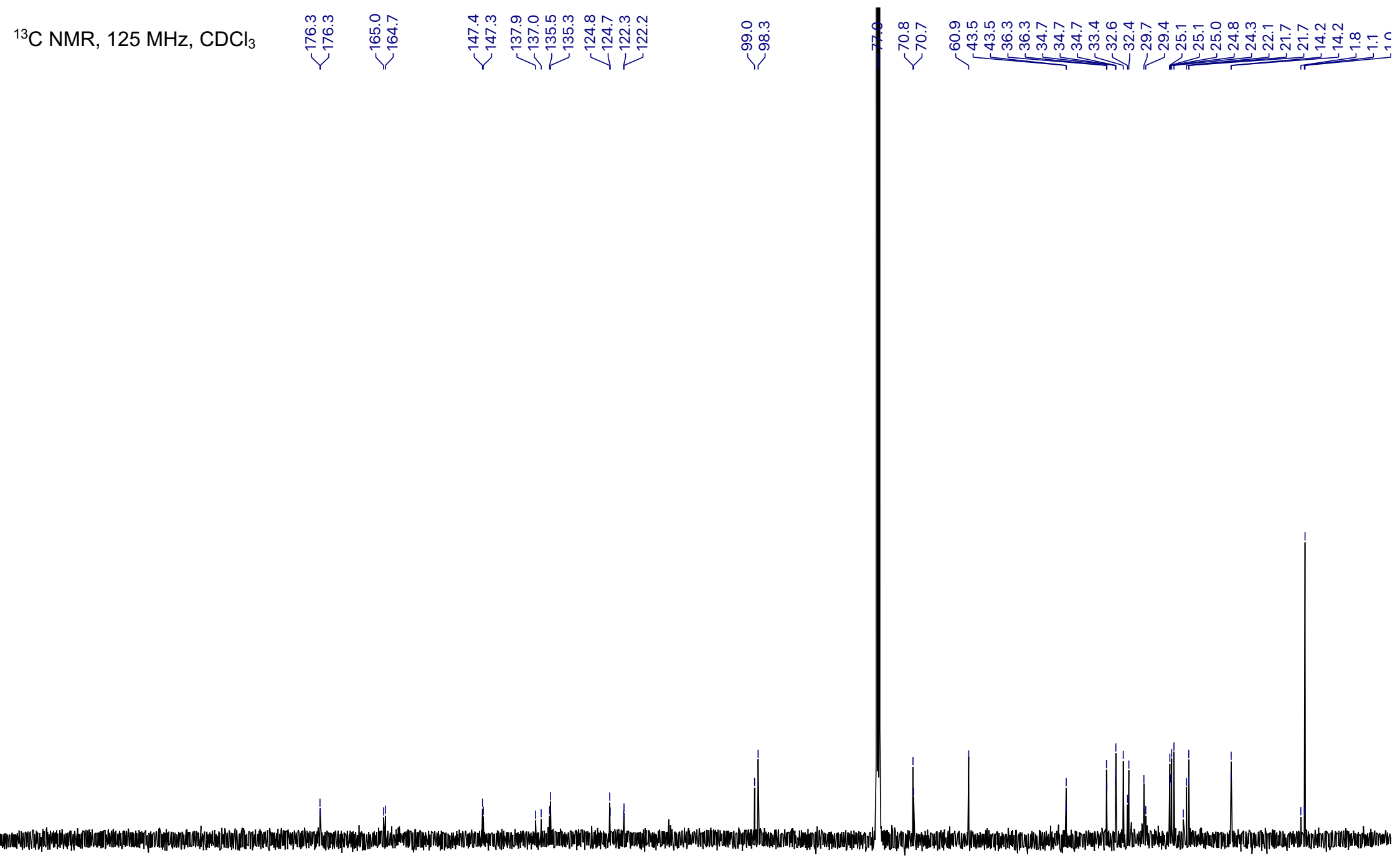

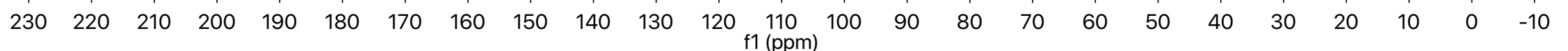


${ }^{1} \mathrm{H}$ NMR, $500 \mathrm{MHz}, \mathrm{C}_{6} \mathrm{D}_{6}$

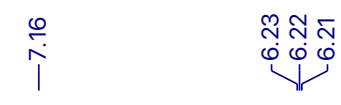

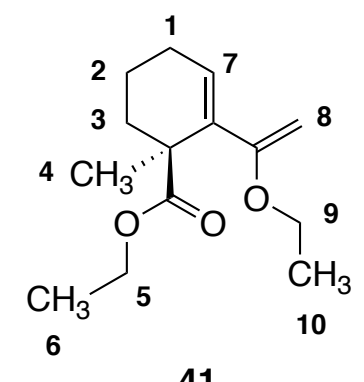

41
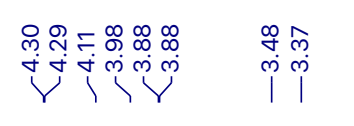

B (d) C (d)

\begin{tabular}{l|l|l|}
\hline 4.29 & 3.88 \\
\hline
\end{tabular}

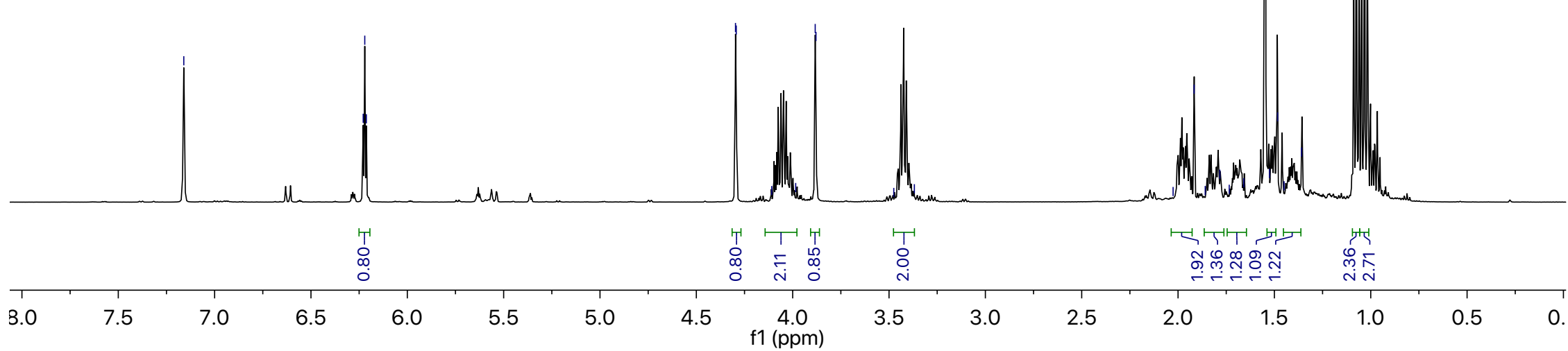




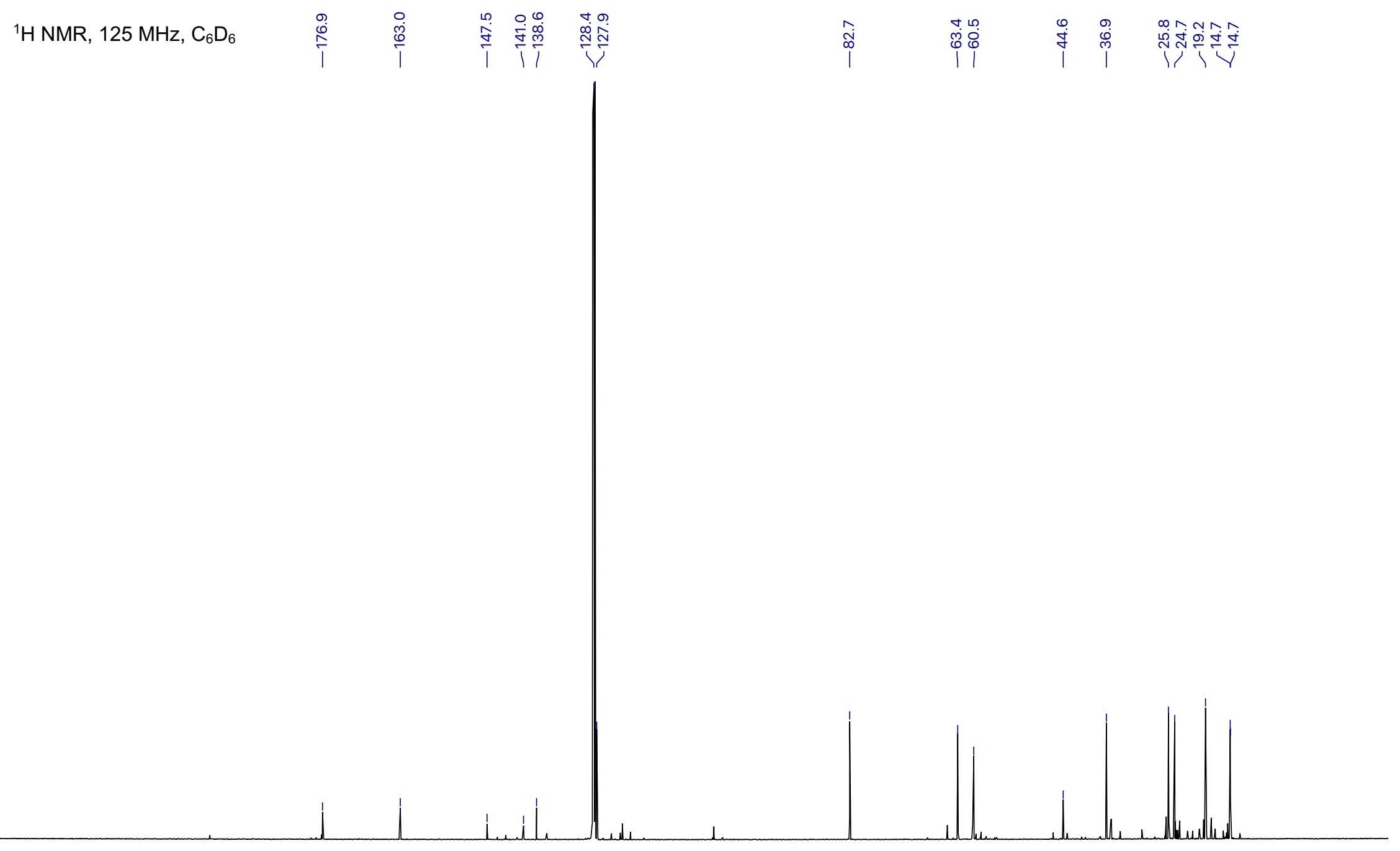

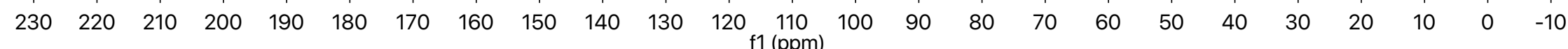




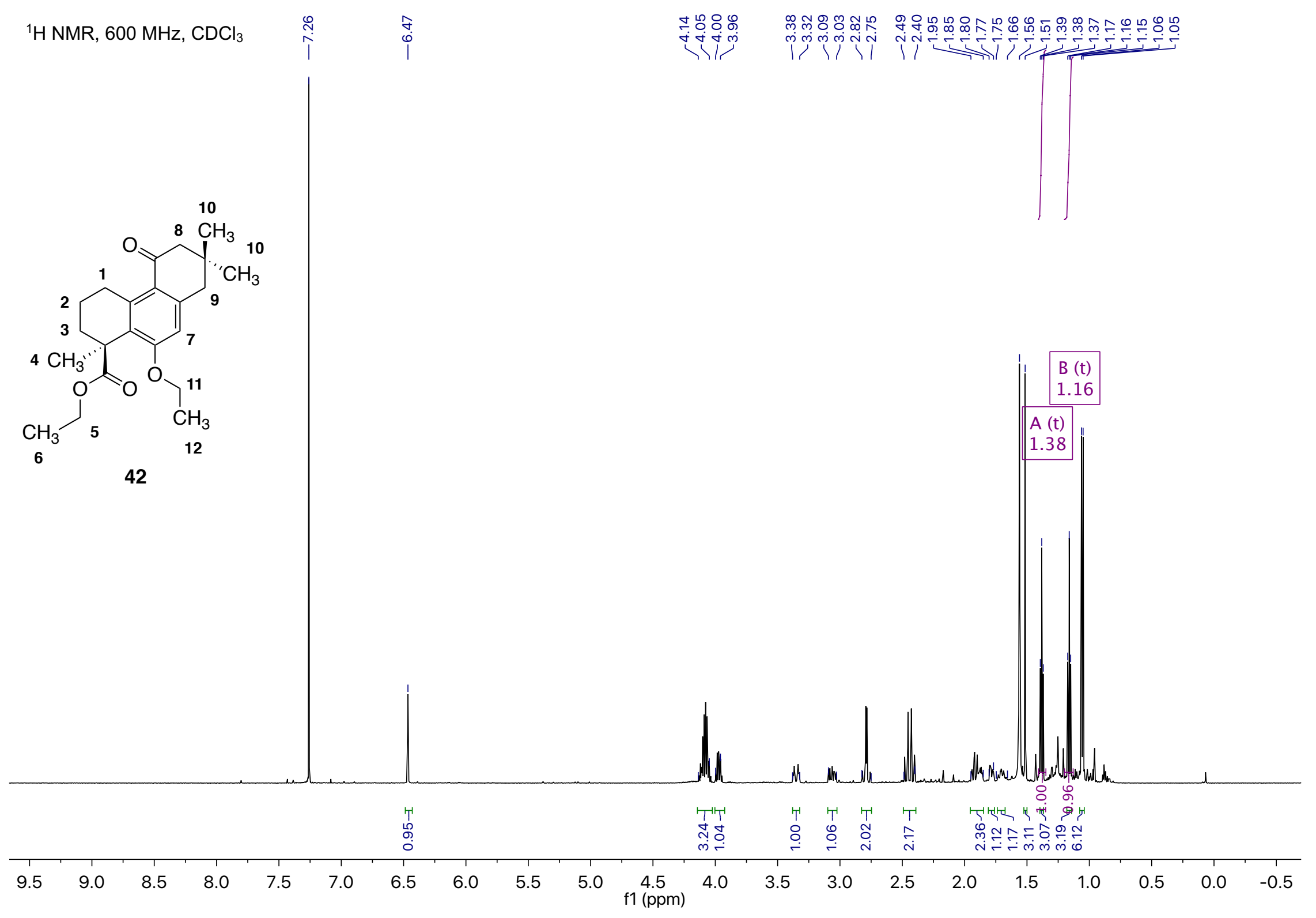


${ }^{13} \mathrm{CNMR}, 150 \mathrm{MHz}, \mathrm{CDCl}_{3}$

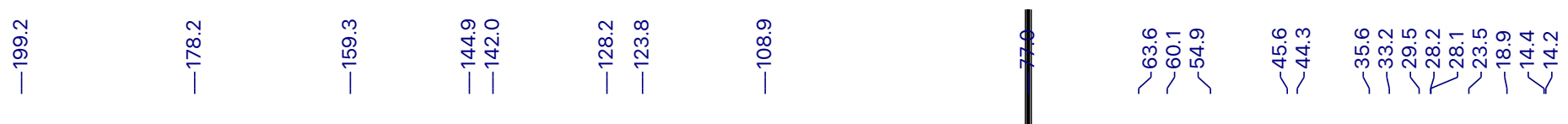

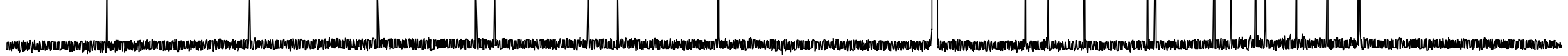

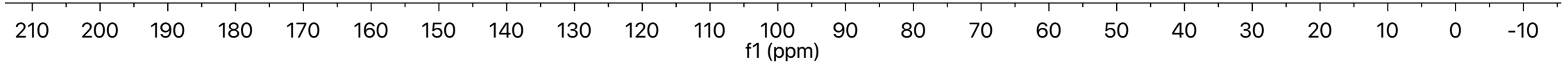


${ }^{1} \mathrm{H} \mathrm{NMR}, 600 \mathrm{MHz}, \mathrm{CDCl}_{3}$

$\stackrel{0}{\stackrel{0}{i}} \stackrel{\substack{n \\ i}}{i}$

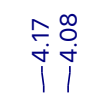

ij
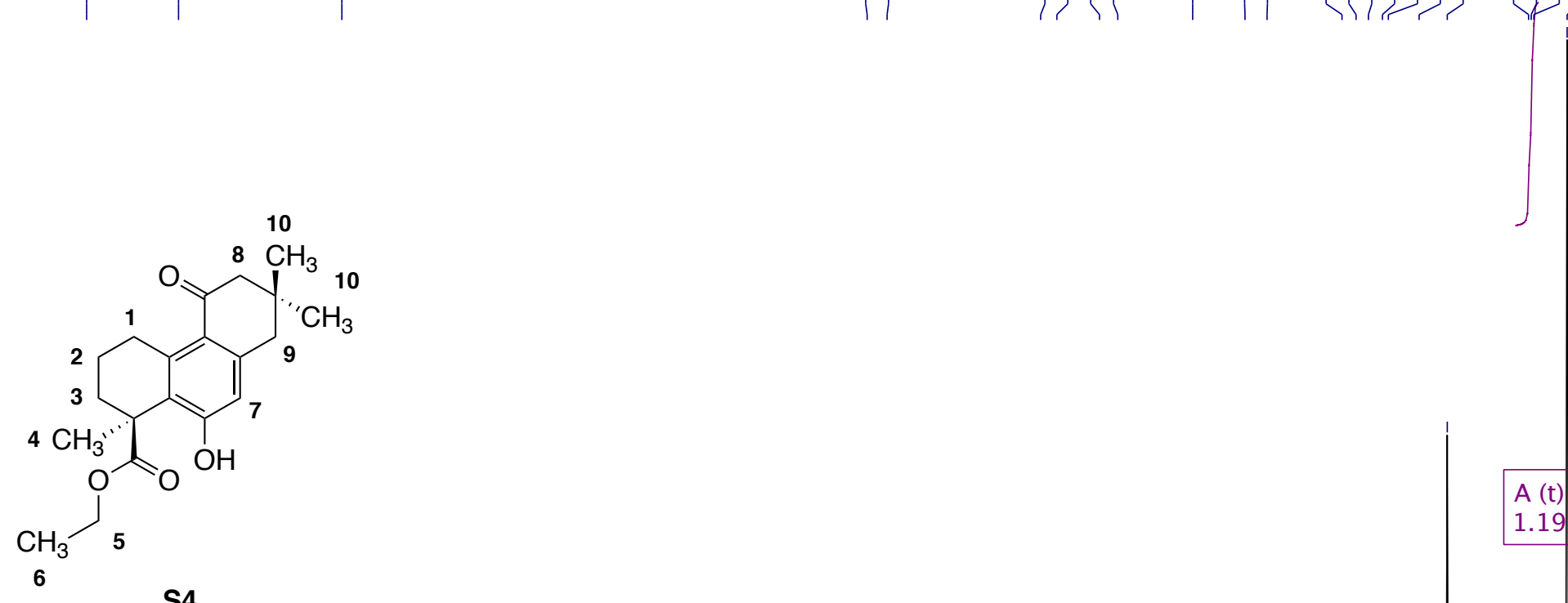

S4

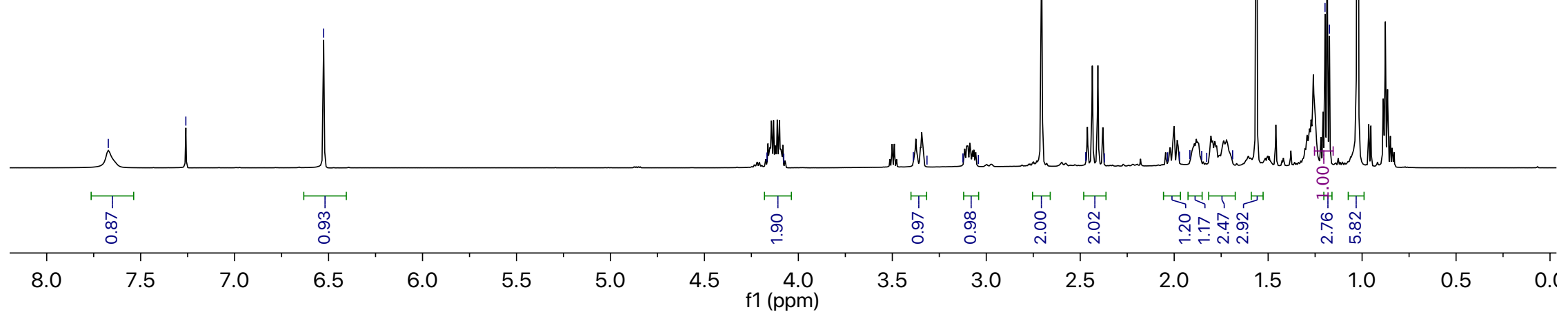



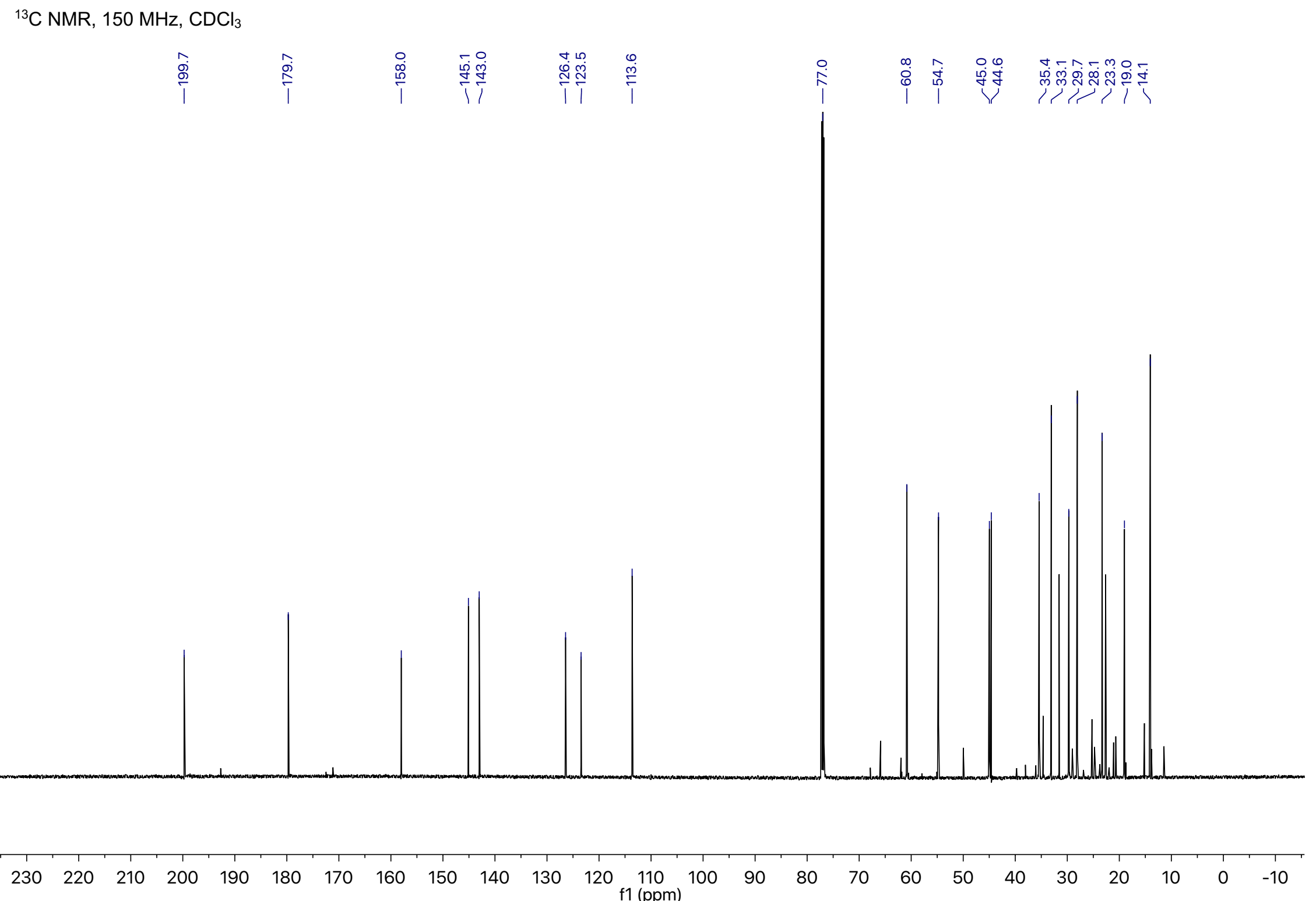


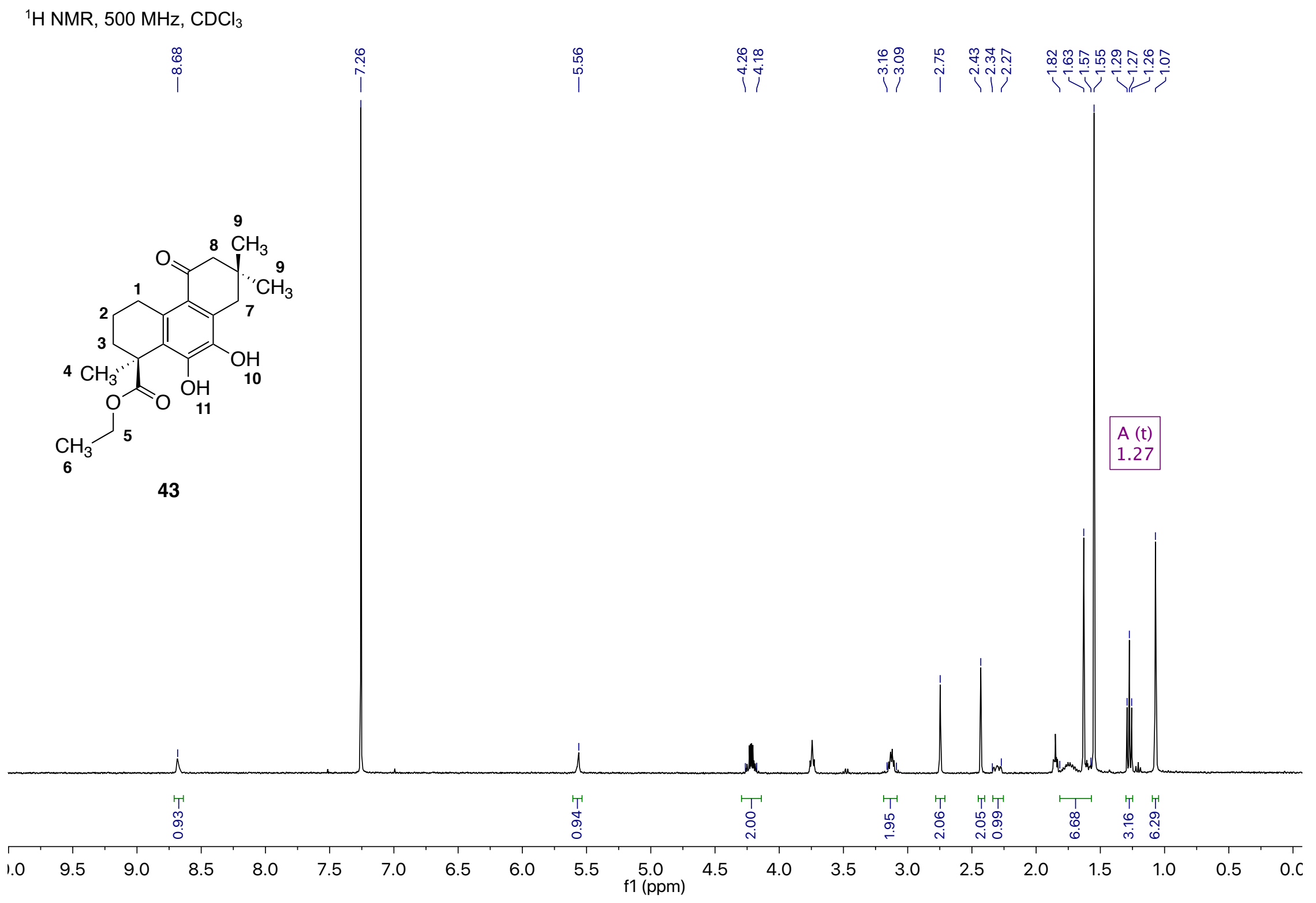


${ }^{13} \mathrm{C} \mathrm{NMR}, 150 \mathrm{MHz}, \mathrm{CDCl}_{3}$

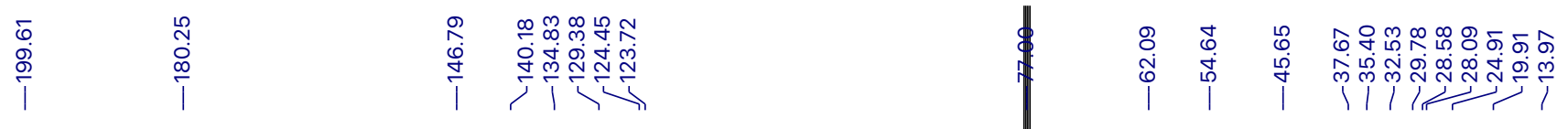

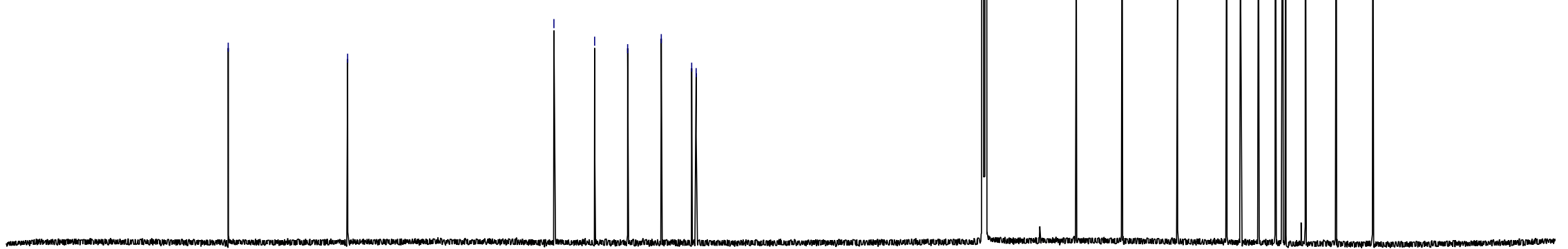

$\begin{array}{lllllllllllllllllllllllll}230 & 220 & 210 & 200 & 190 & 180 & 170 & 160 & 150 & 140 & 130 & 120 & \begin{array}{c}110 \\ \mathrm{f}(\mathrm{ppm})\end{array} & 100 & 90 & 80 & 70 & 60 & 50 & 40 & 30 & 20 & 10 & 0 & -10\end{array}$ 


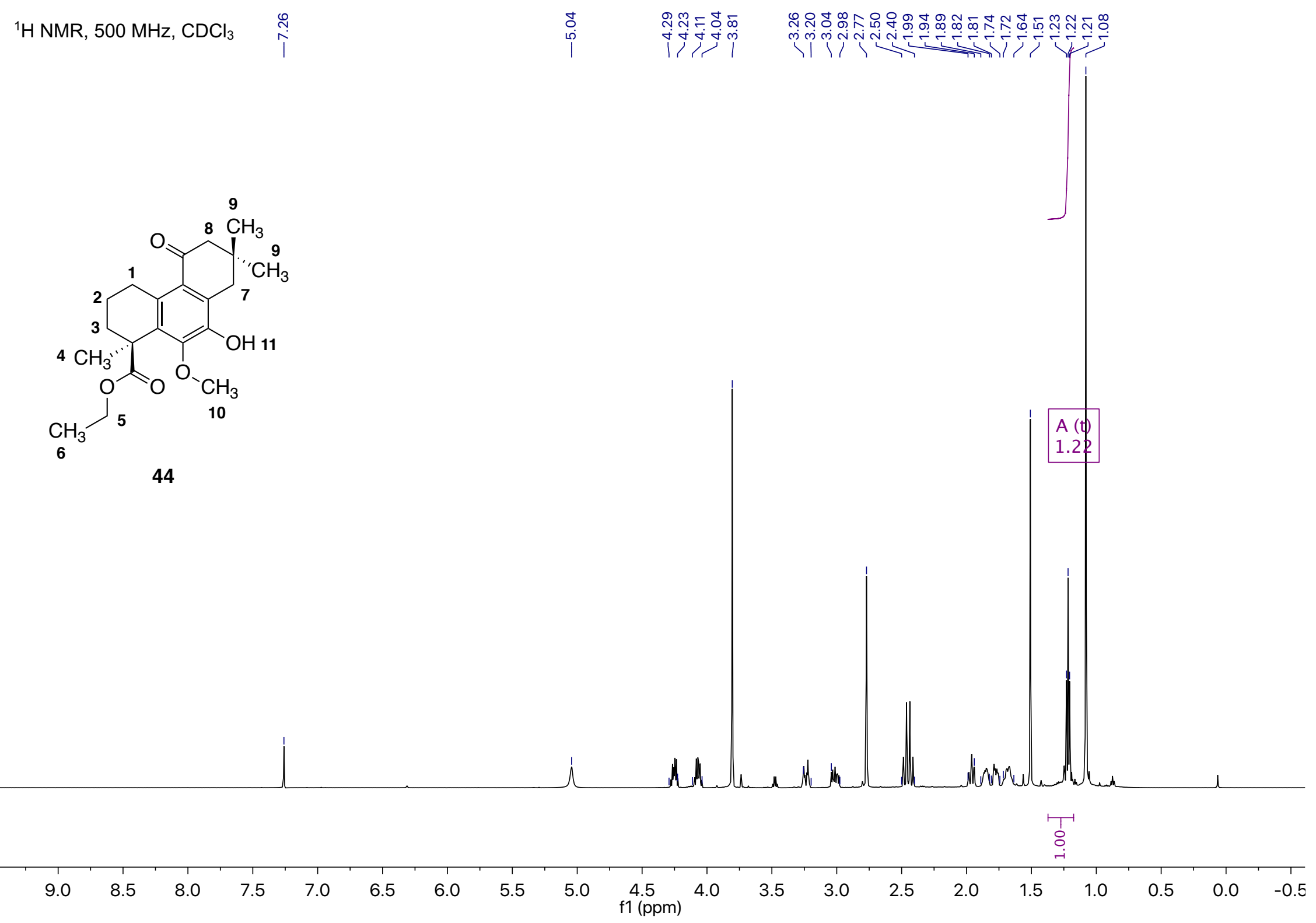



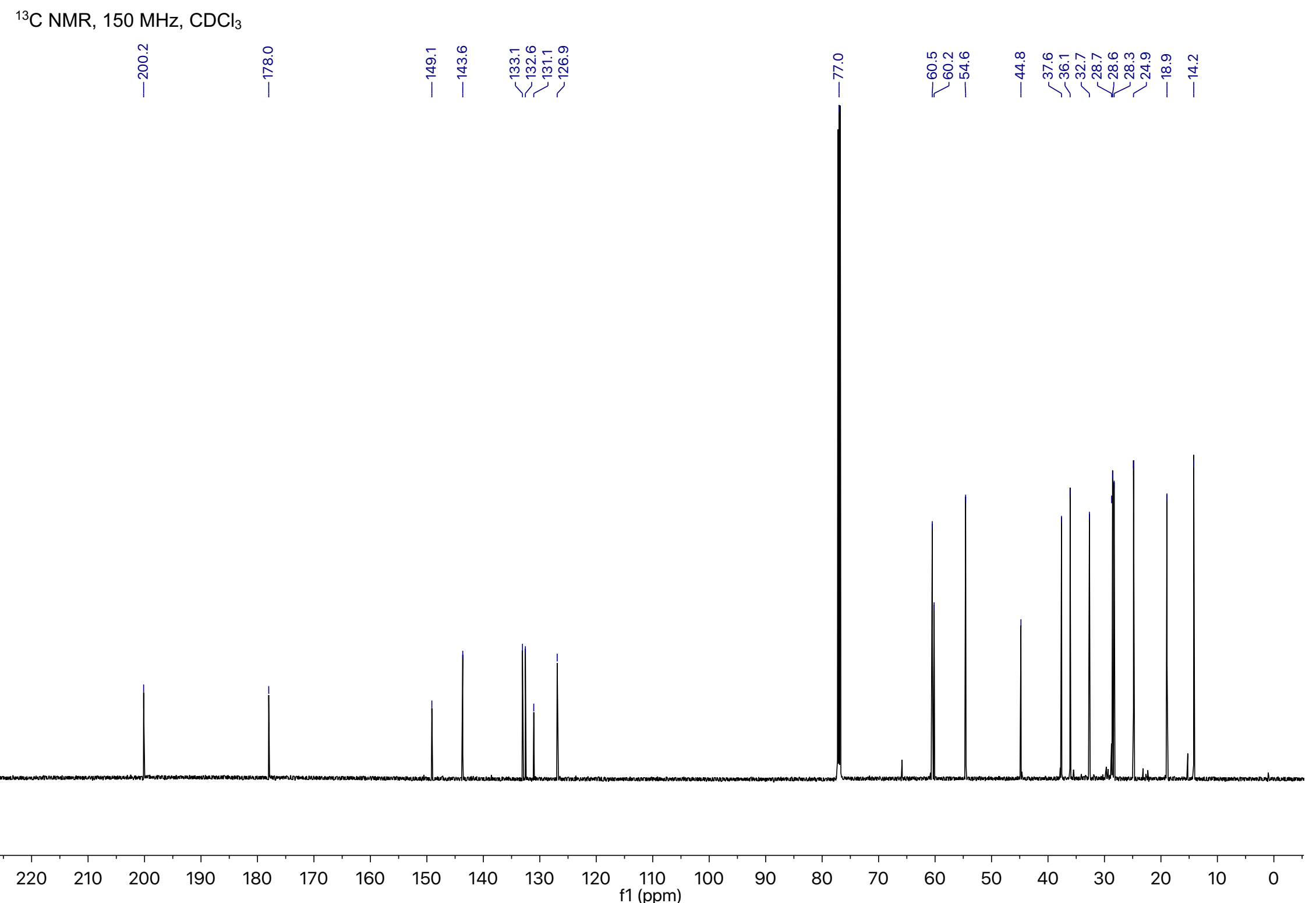


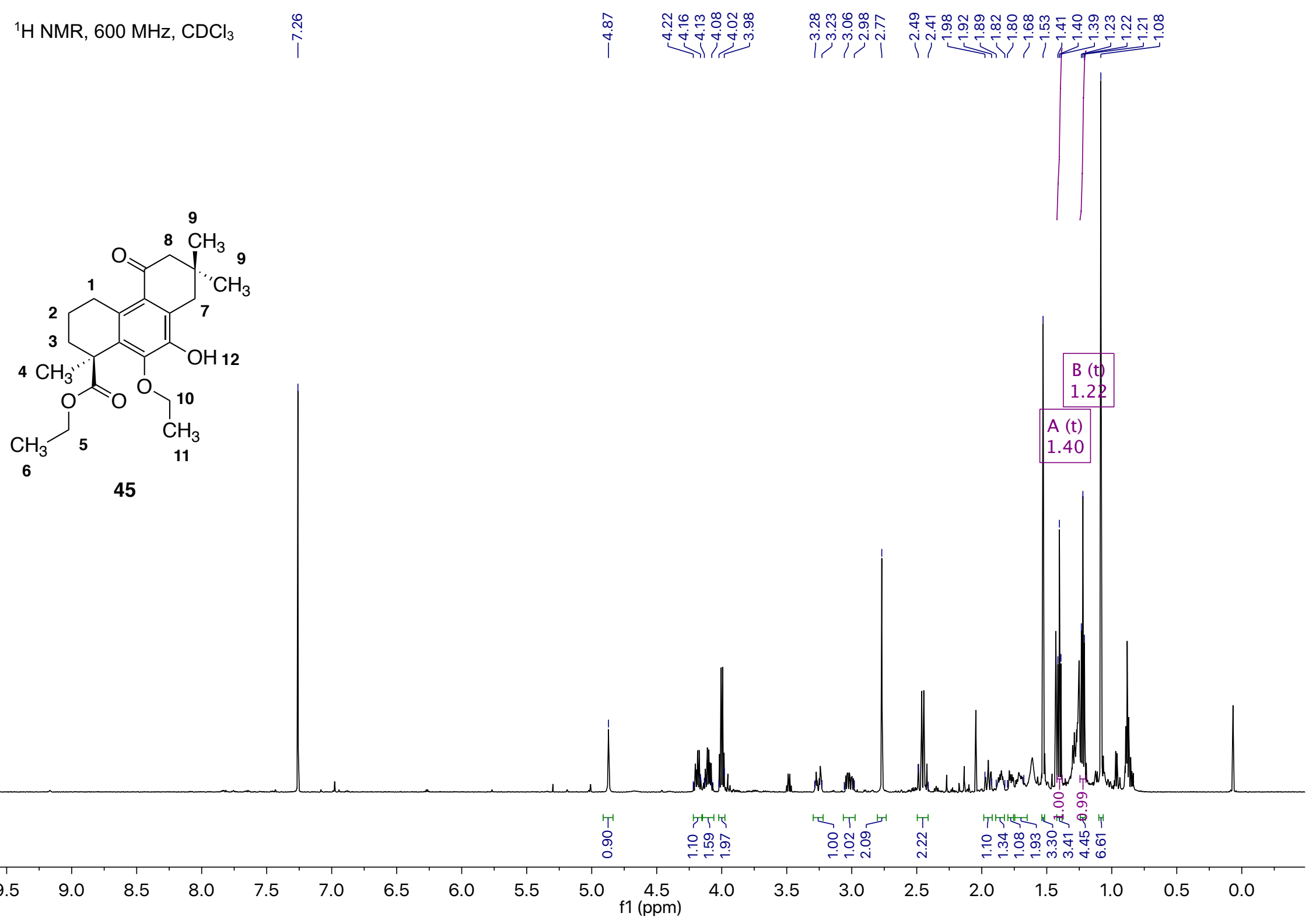




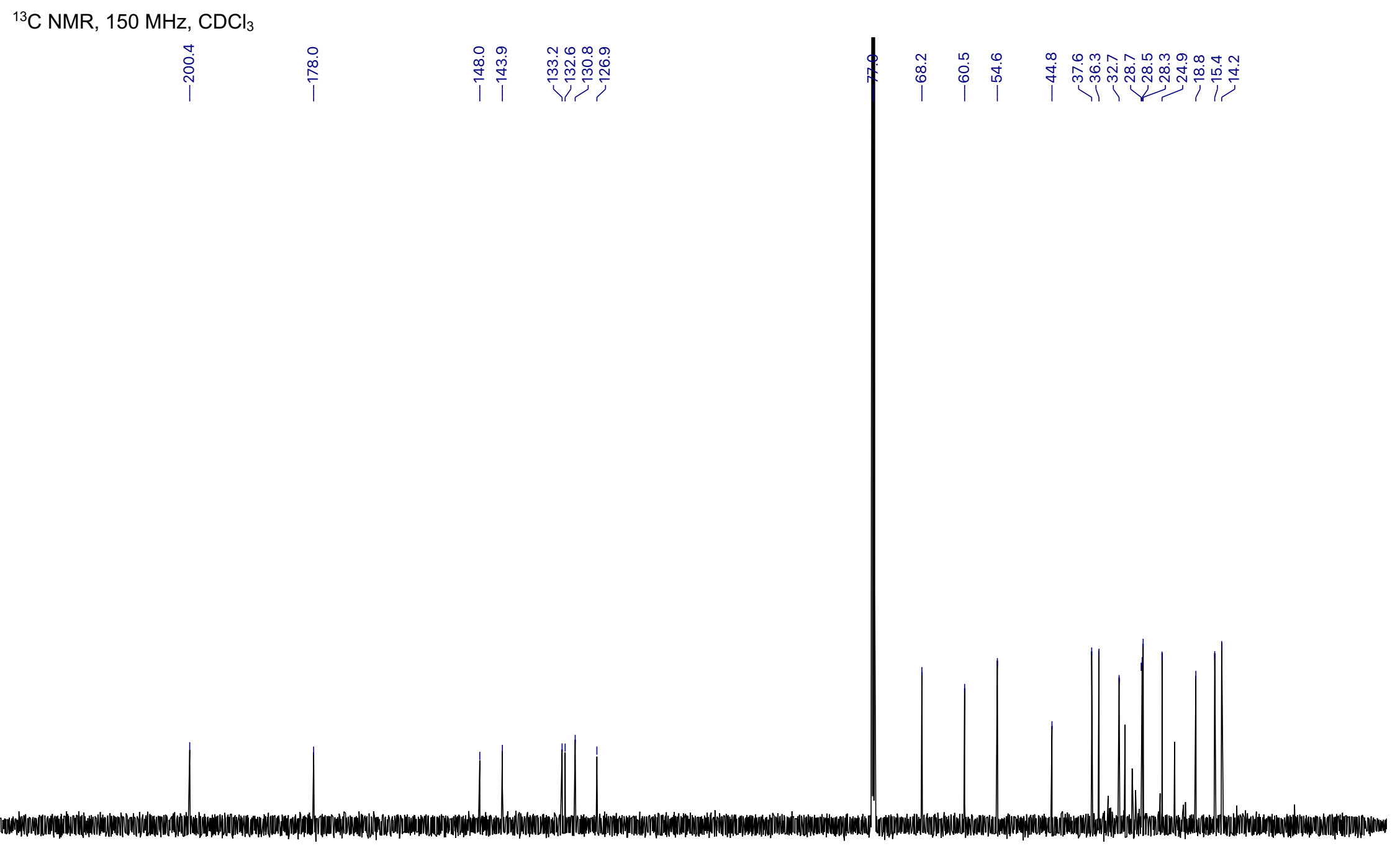

$\begin{array}{lllllllllllllllllllllllll}230 & 220 & 210 & 200 & 190 & 180 & 170 & 160 & 150 & 140 & 130 & 120 & \begin{array}{c}110 \\ \mathrm{f} 1(\mathrm{ppm})\end{array} & 100 & 90 & 80 & 70 & 60 & 50 & 40 & 30 & 20 & 10 & 0 & -10\end{array}$ 


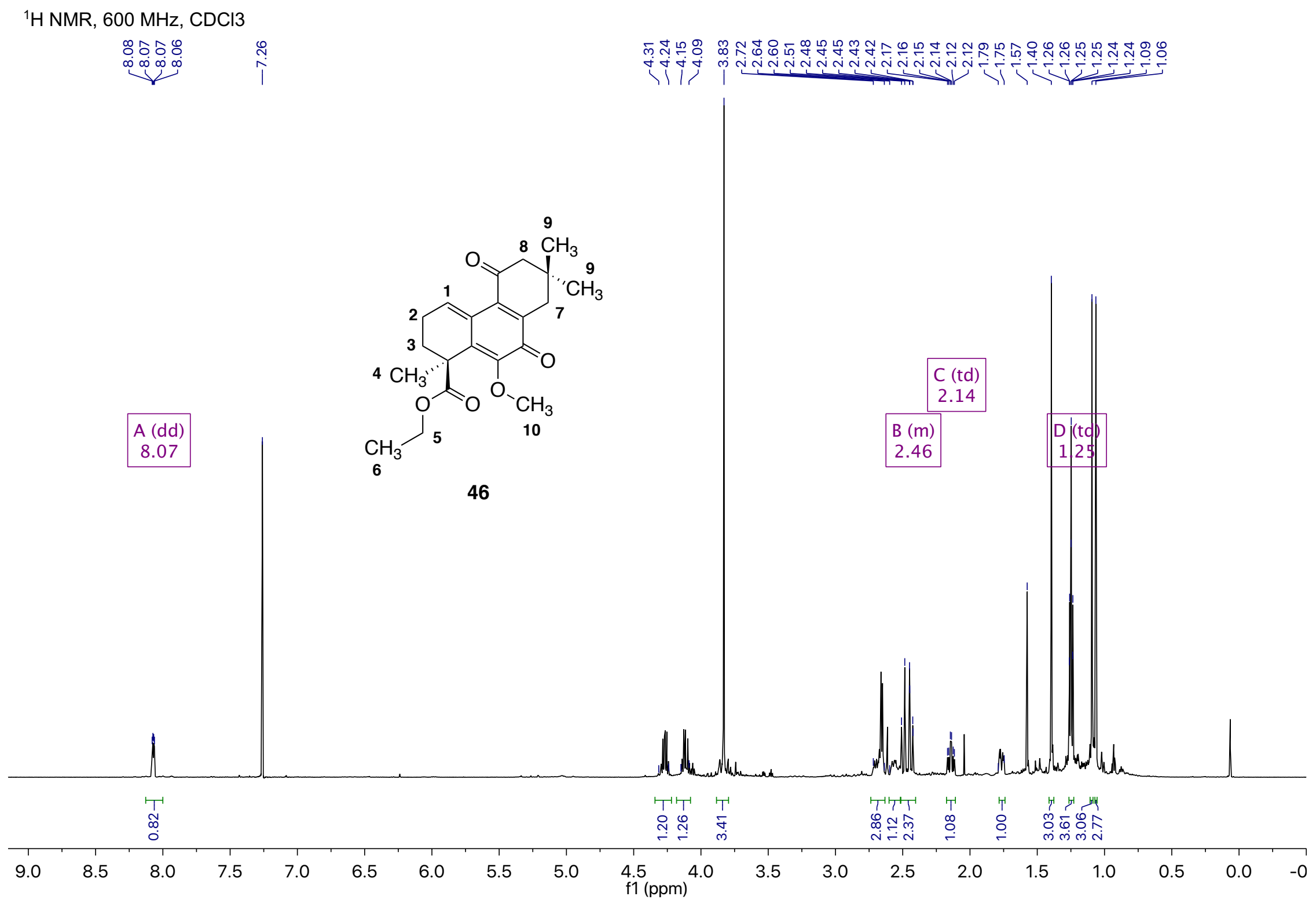


${ }^{1} \mathrm{H}$ NMR, $125 \mathrm{MHz}, \mathrm{CDCl} 3$

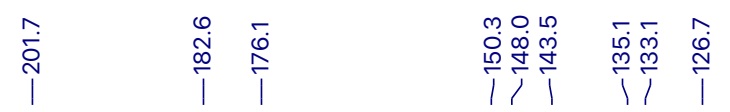

\$ إن

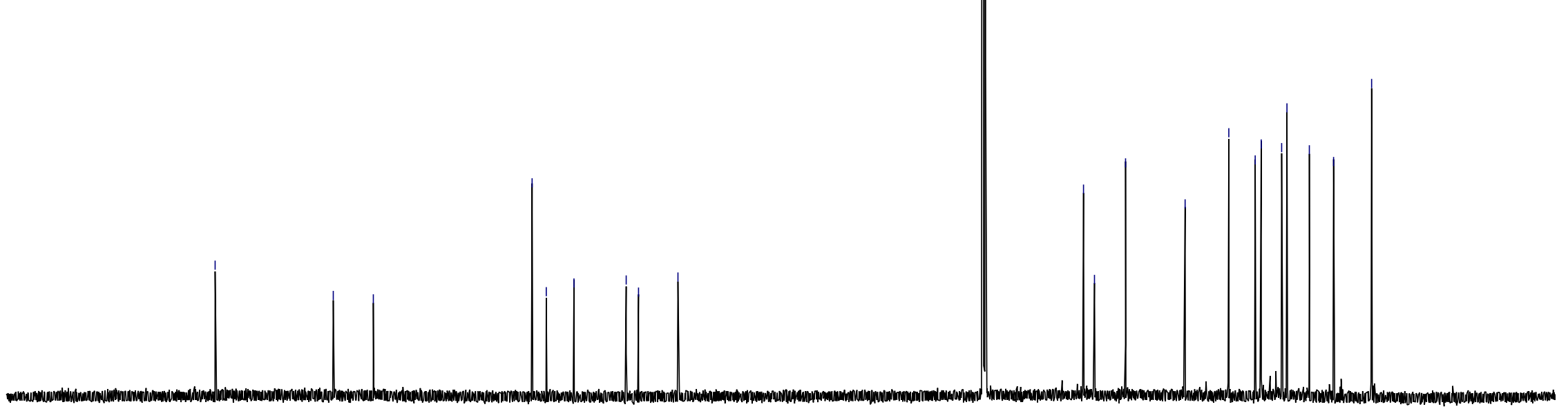

$\begin{array}{llll}230 & 220 & 210 \quad 200\end{array}$

190

$180 \quad 170$

160

150

130

$120 \quad 110$

f1 (ppm)

90

$80 \quad 70$

60

50

40

30

20

$10 \quad 0 \quad-10$ 
${ }^{1} \mathrm{H}$ NMR, $600 \mathrm{MHz}, \mathrm{CDCl} 3$

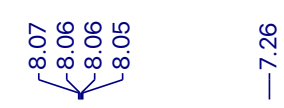

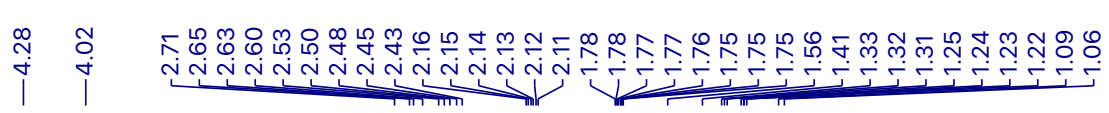

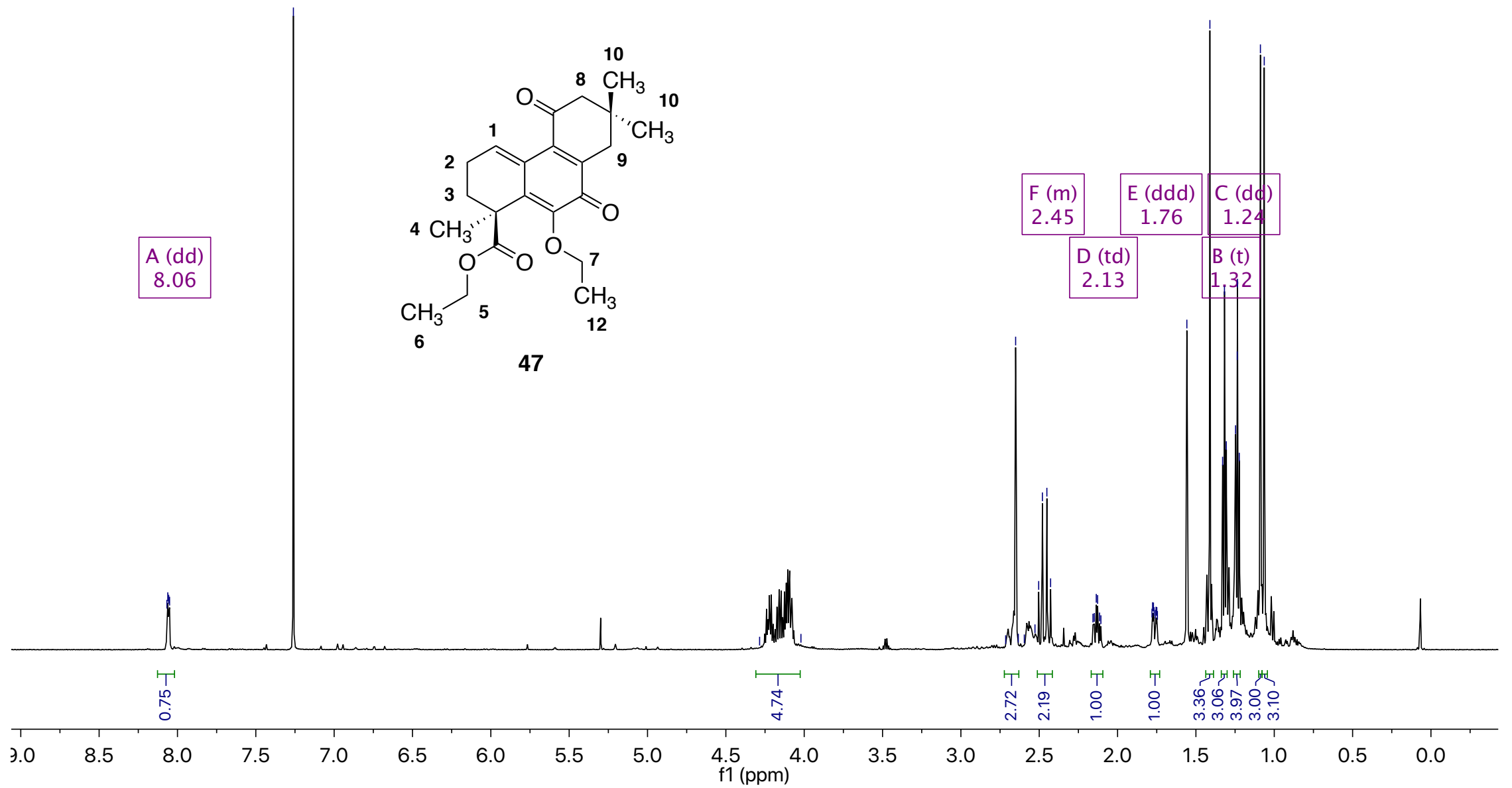


${ }^{1} \mathrm{H}$ NMR, $125 \mathrm{MHz}, \mathrm{CDCl} 3$

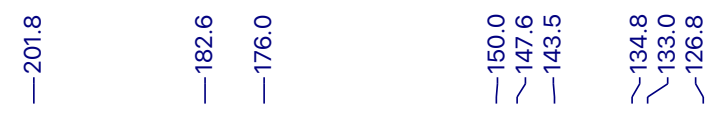

l)

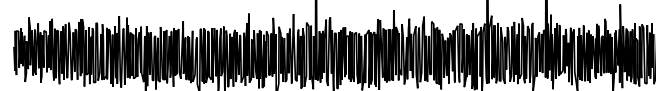

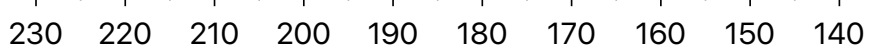

140

30

f1 (ppm)

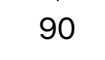

80

60

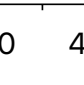

30

$20 \quad 10$

$0-10$ 
${ }^{1} \mathrm{H} \mathrm{NMR}, 600 \mathrm{MHz}, \mathrm{CDCl}_{3}$

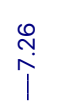

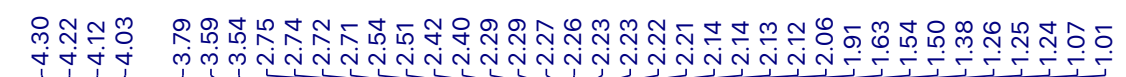

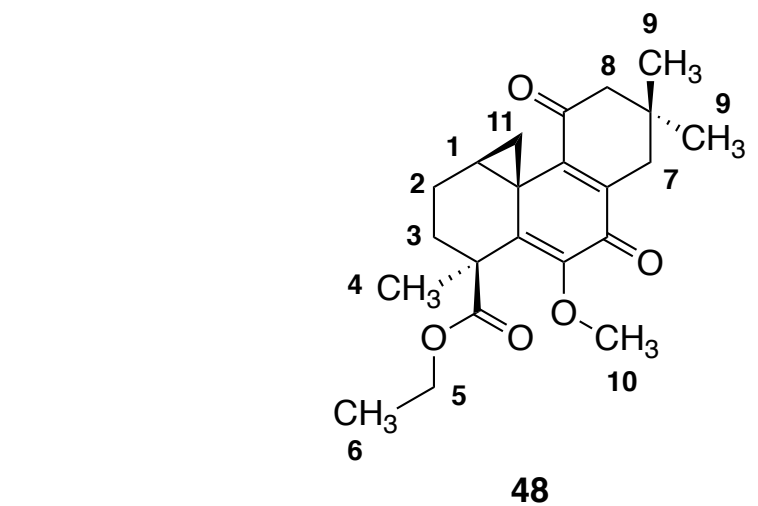

111111

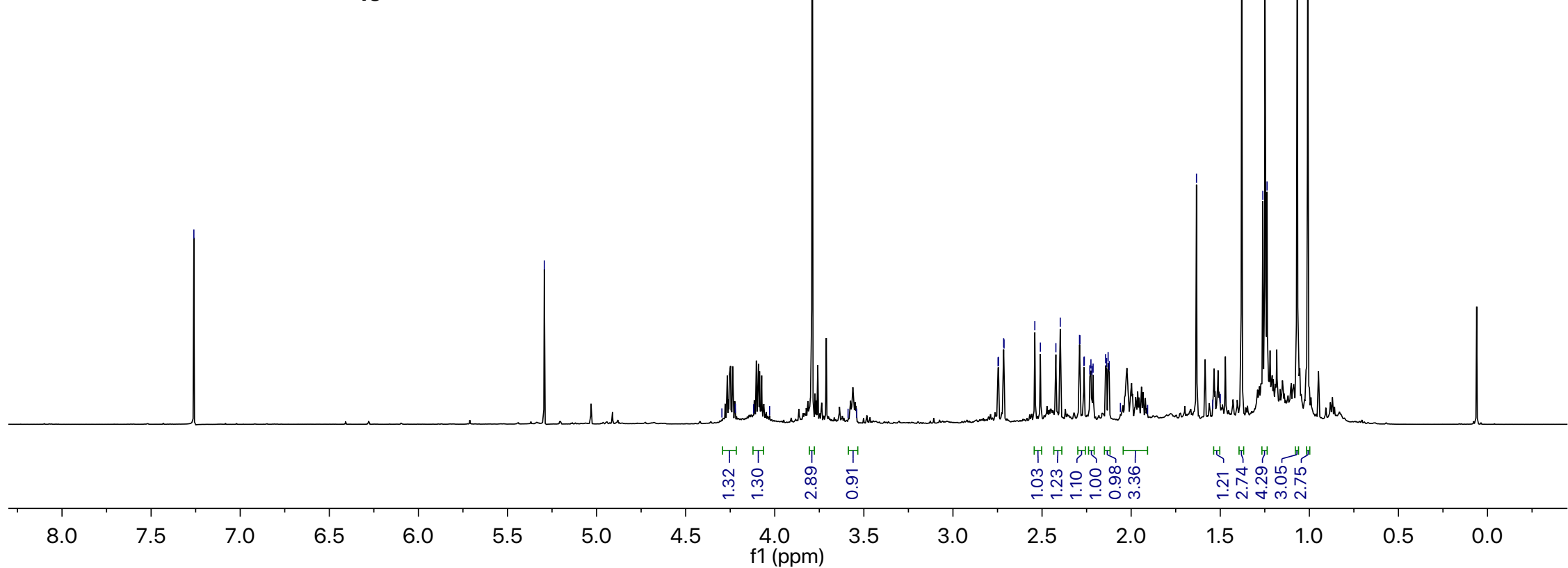




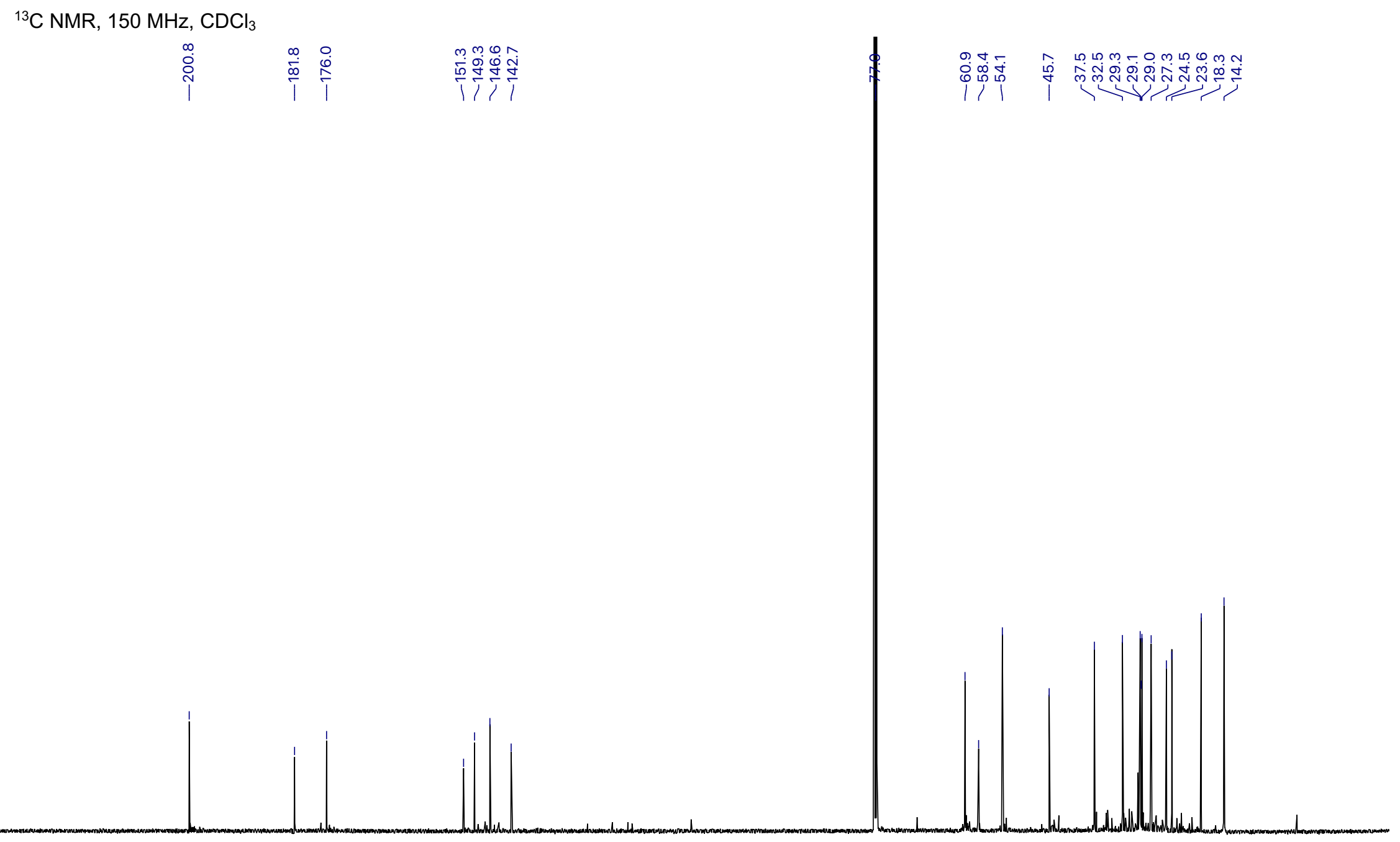

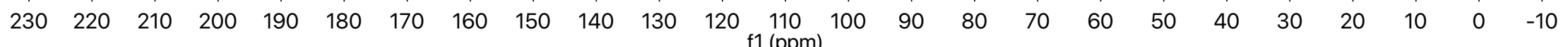




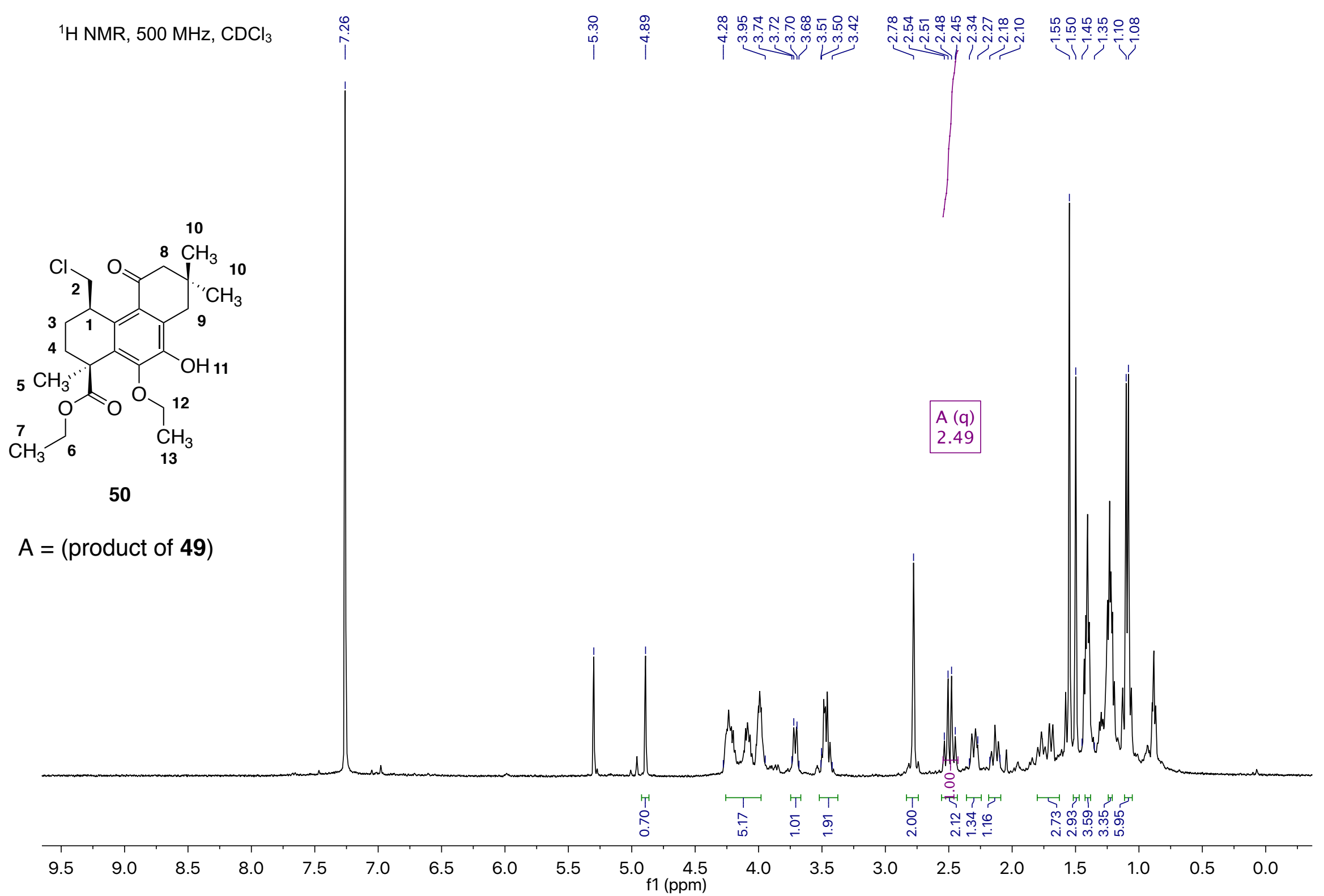




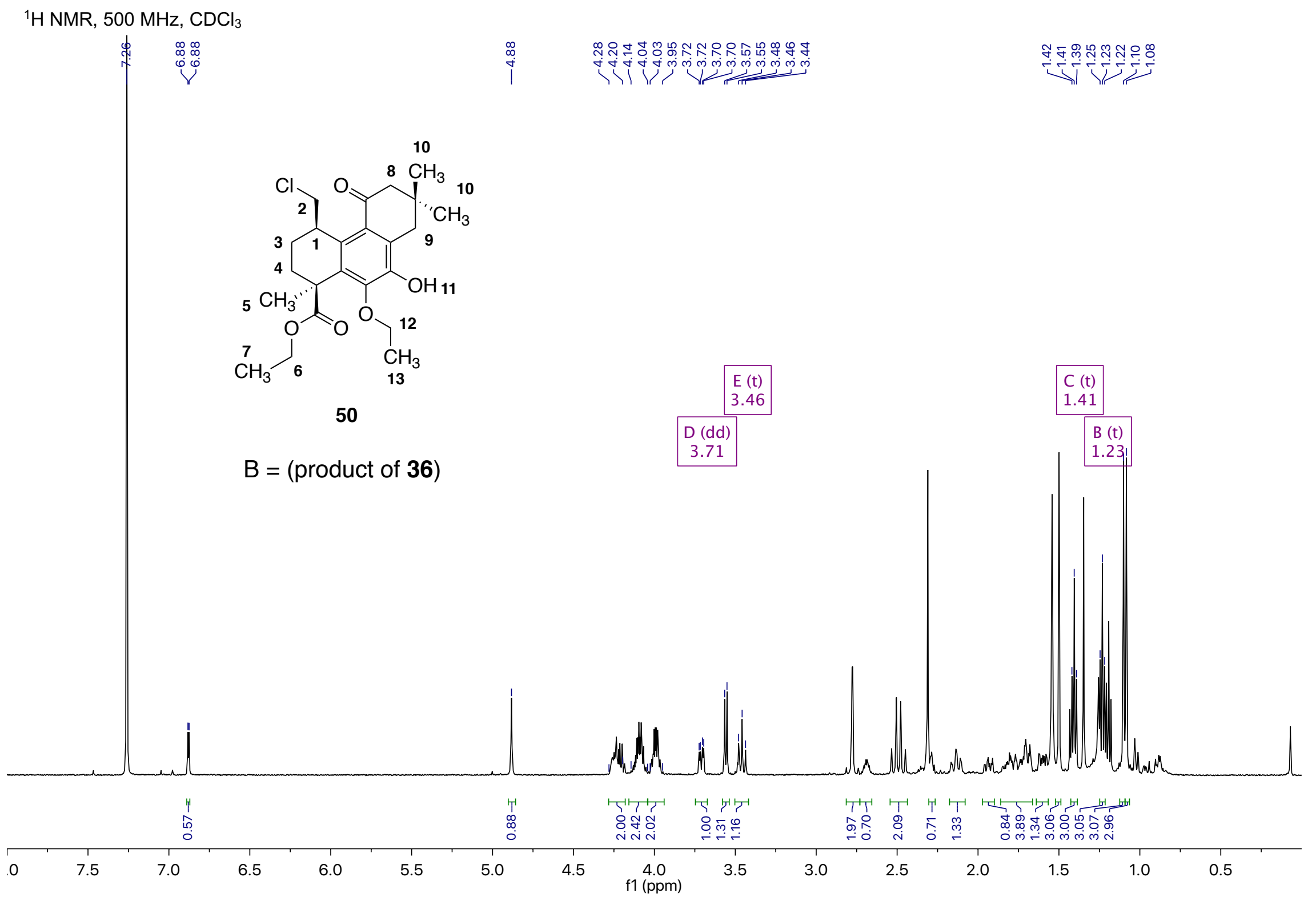


${ }^{1} \mathrm{H} \mathrm{NMR}, 500 \mathrm{MHz}, \mathrm{CDCl}_{3}$

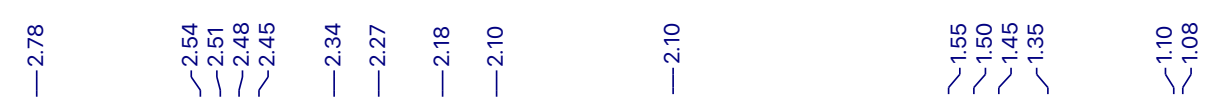
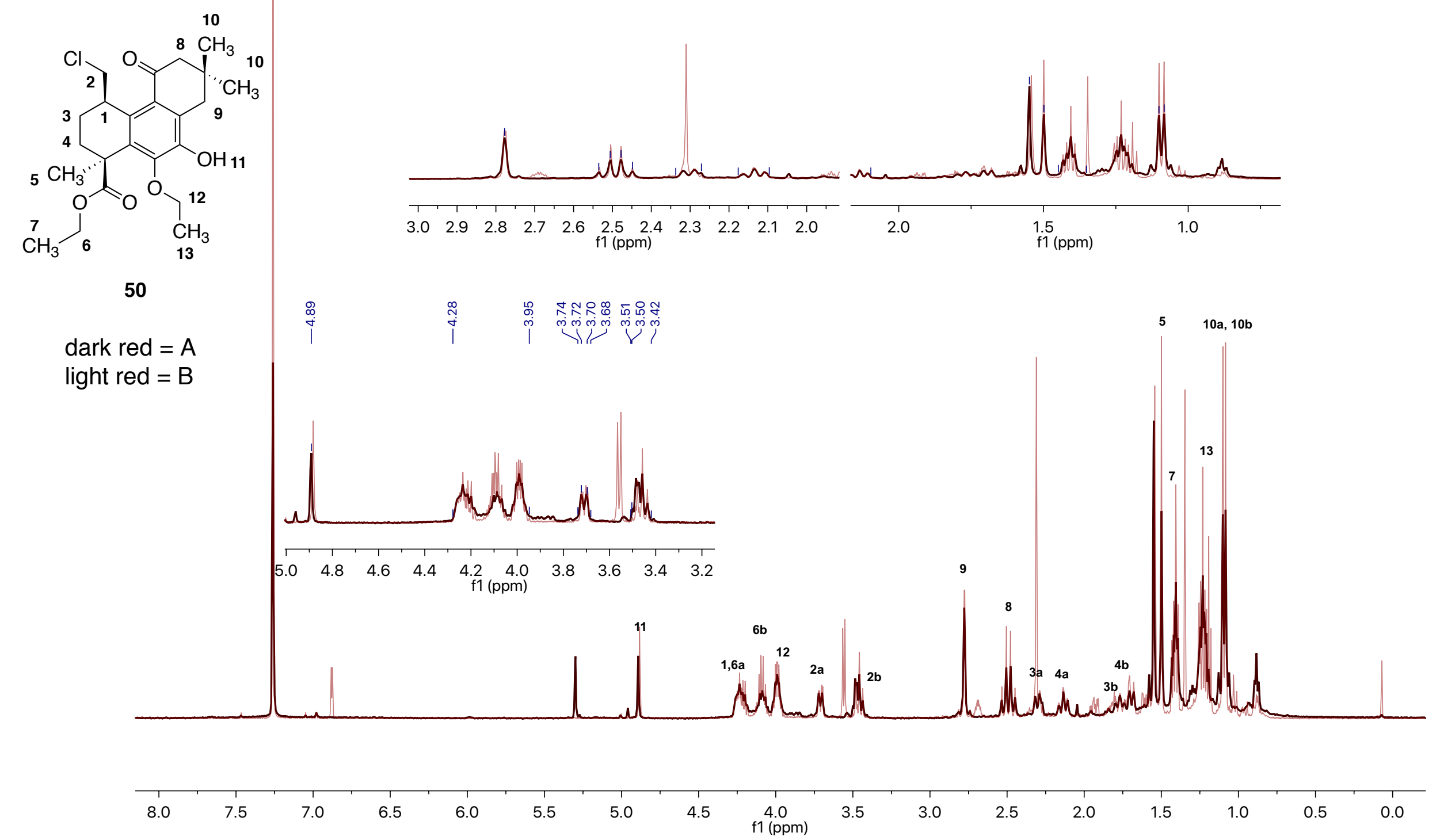
${ }^{13} \mathrm{C} \mathrm{NMR}, 125 \mathrm{MHz}, \mathrm{CDCl}_{3}$
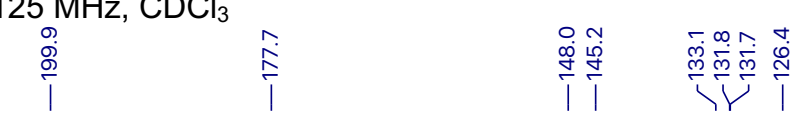

\$
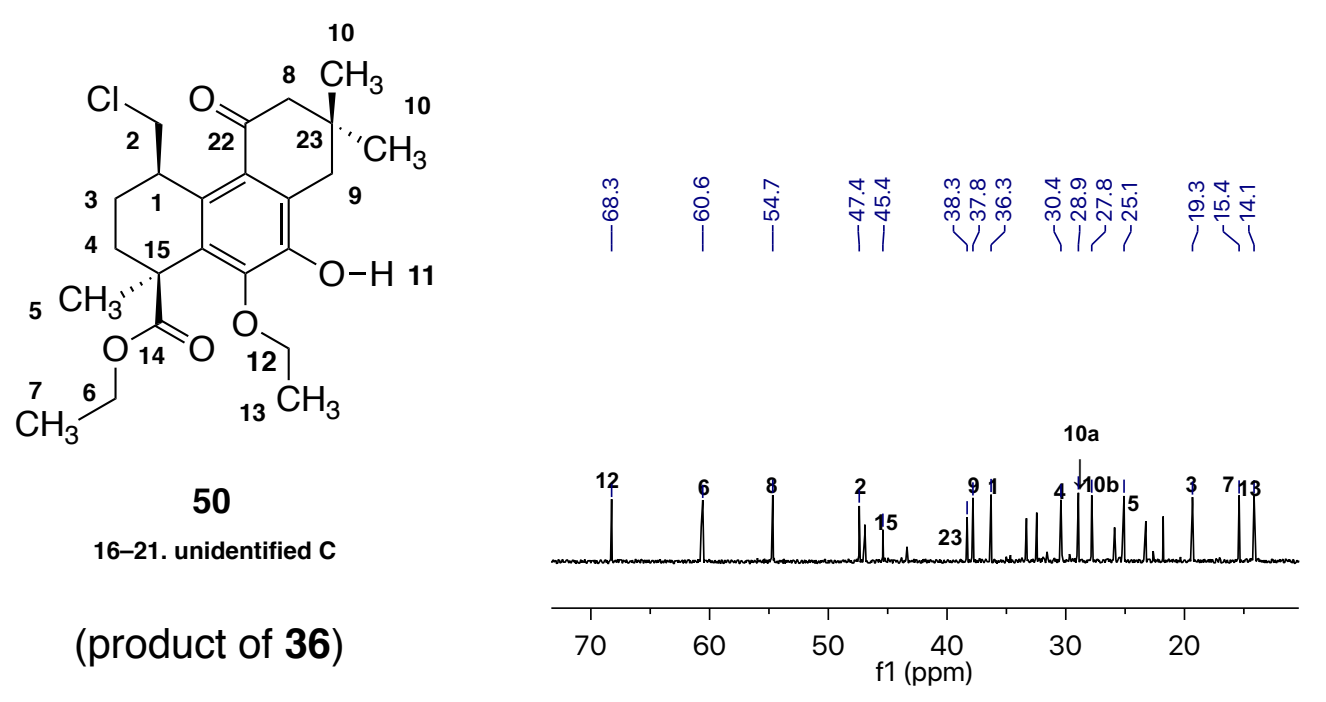

(product of 36)

6-21

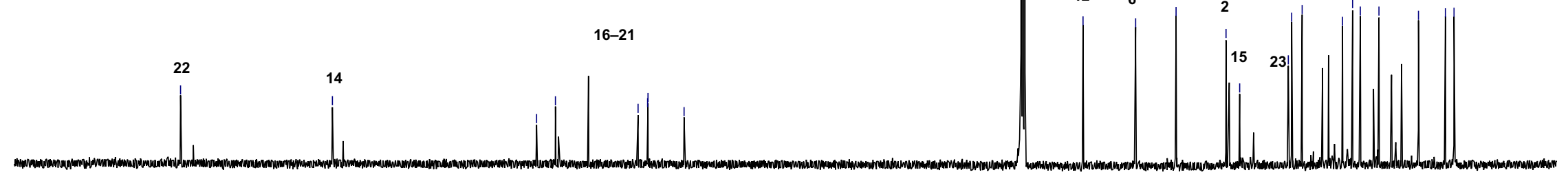

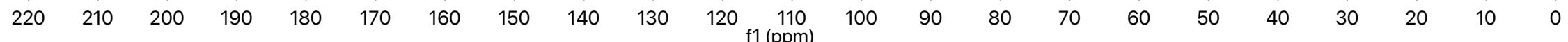




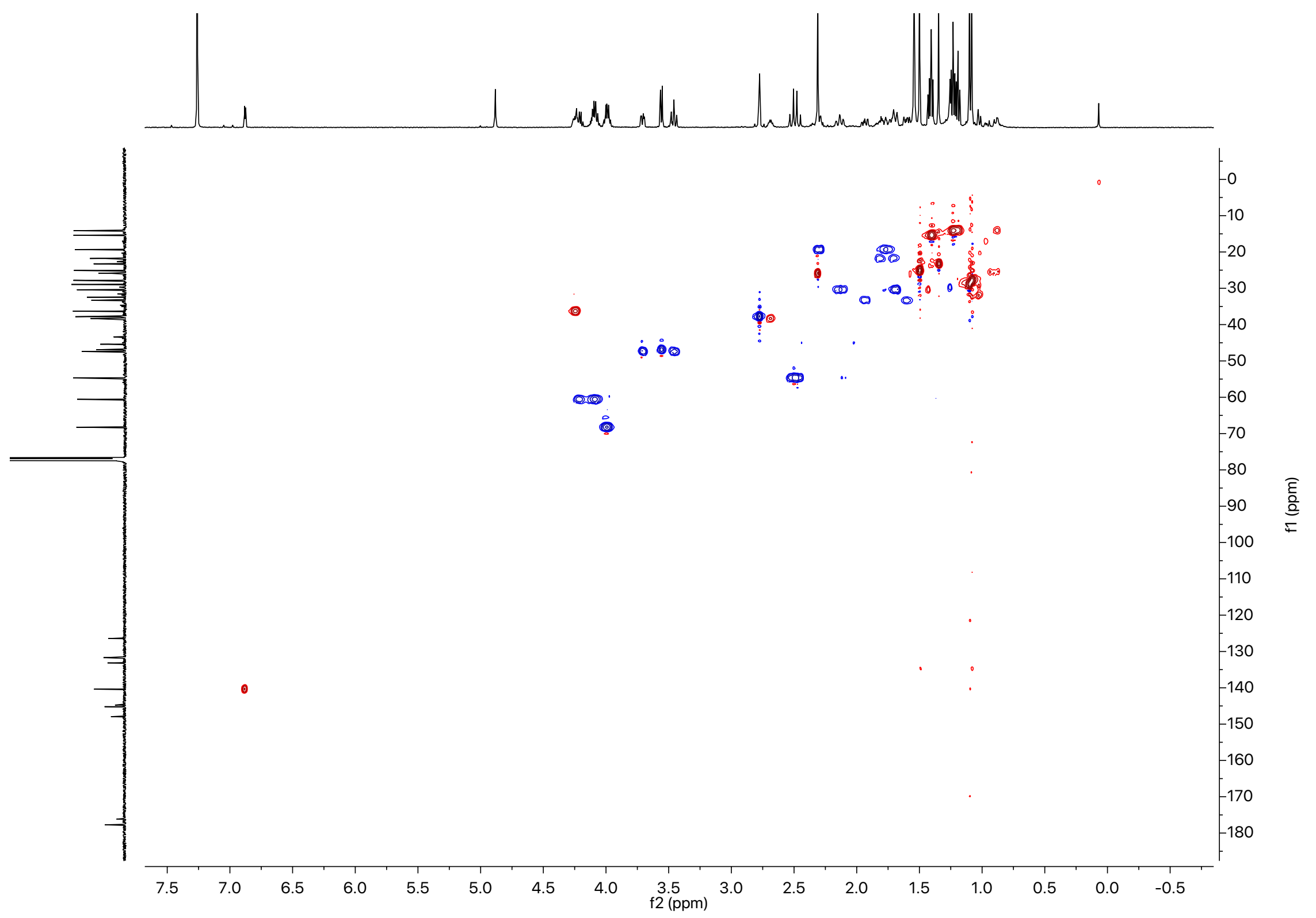




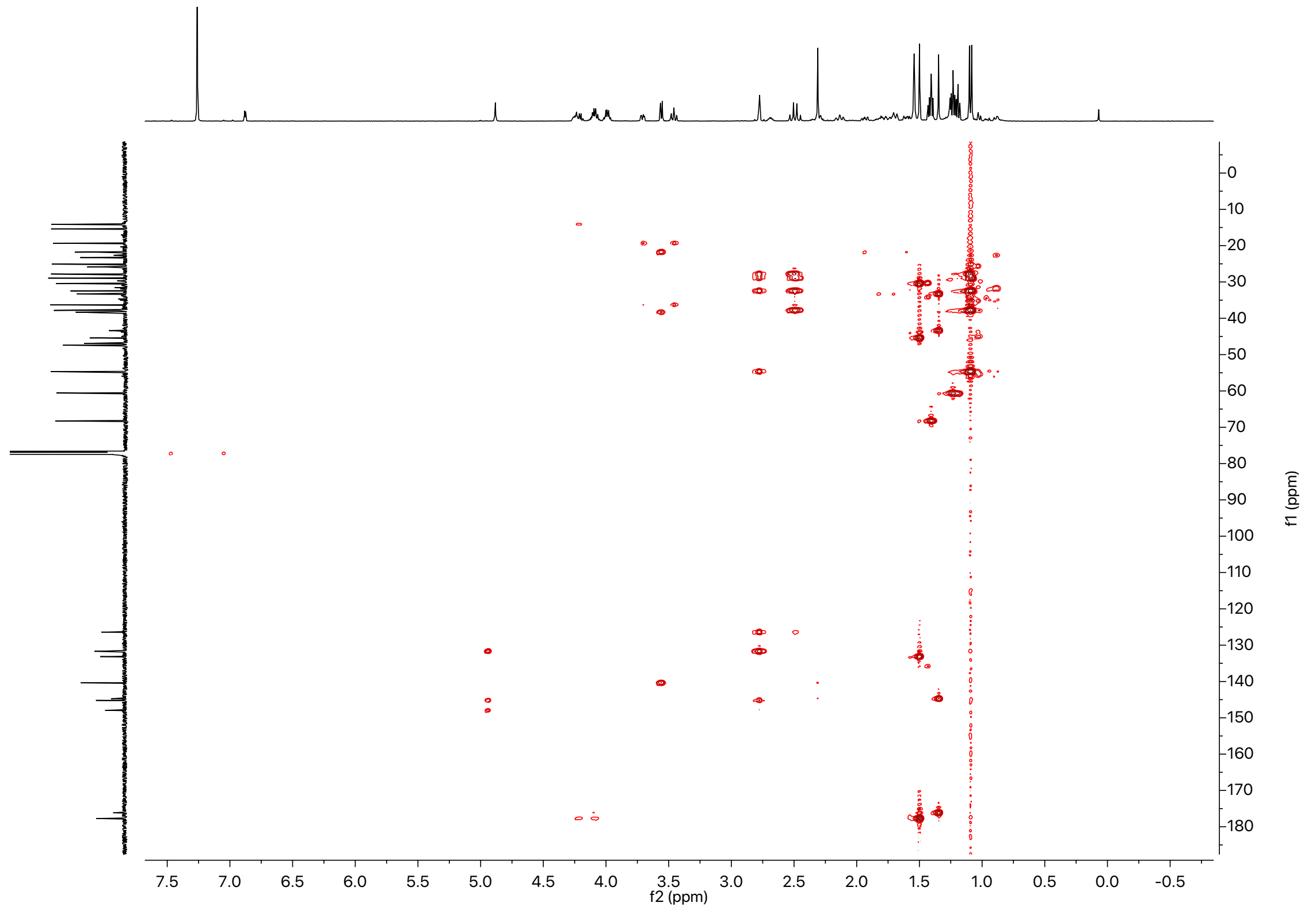




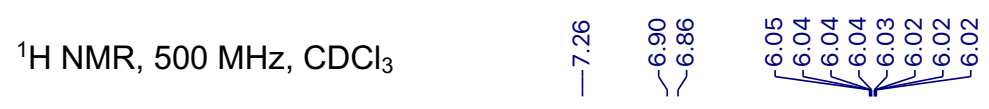
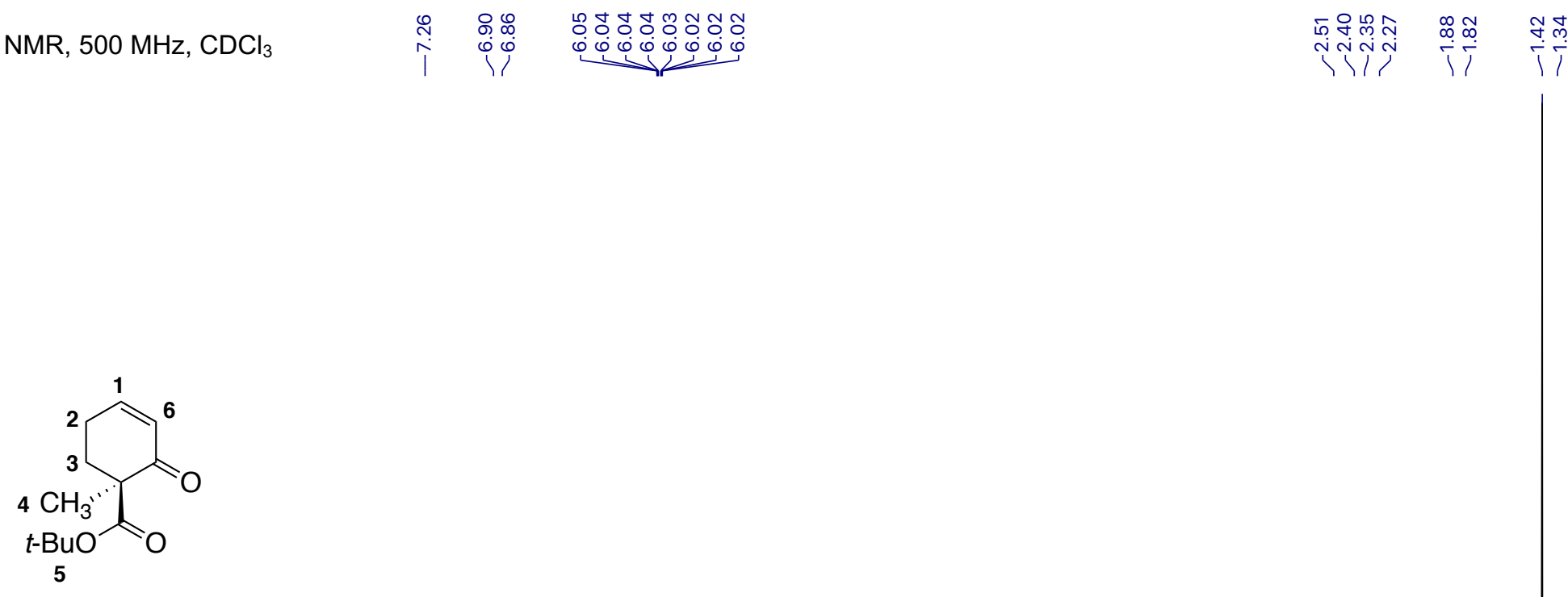

63

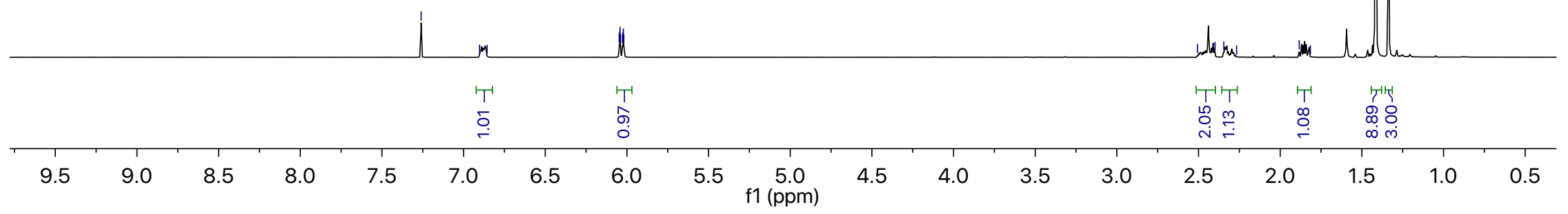


${ }^{13} \mathrm{C}$ NMR, $125 \mathrm{MHz}, \mathrm{CDCl}_{3}$

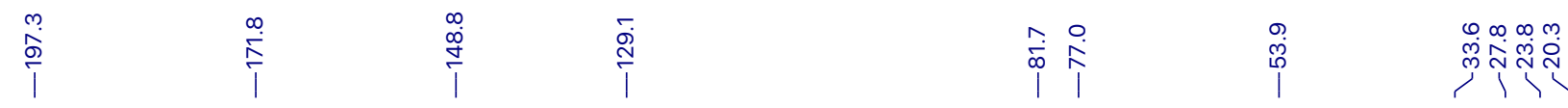

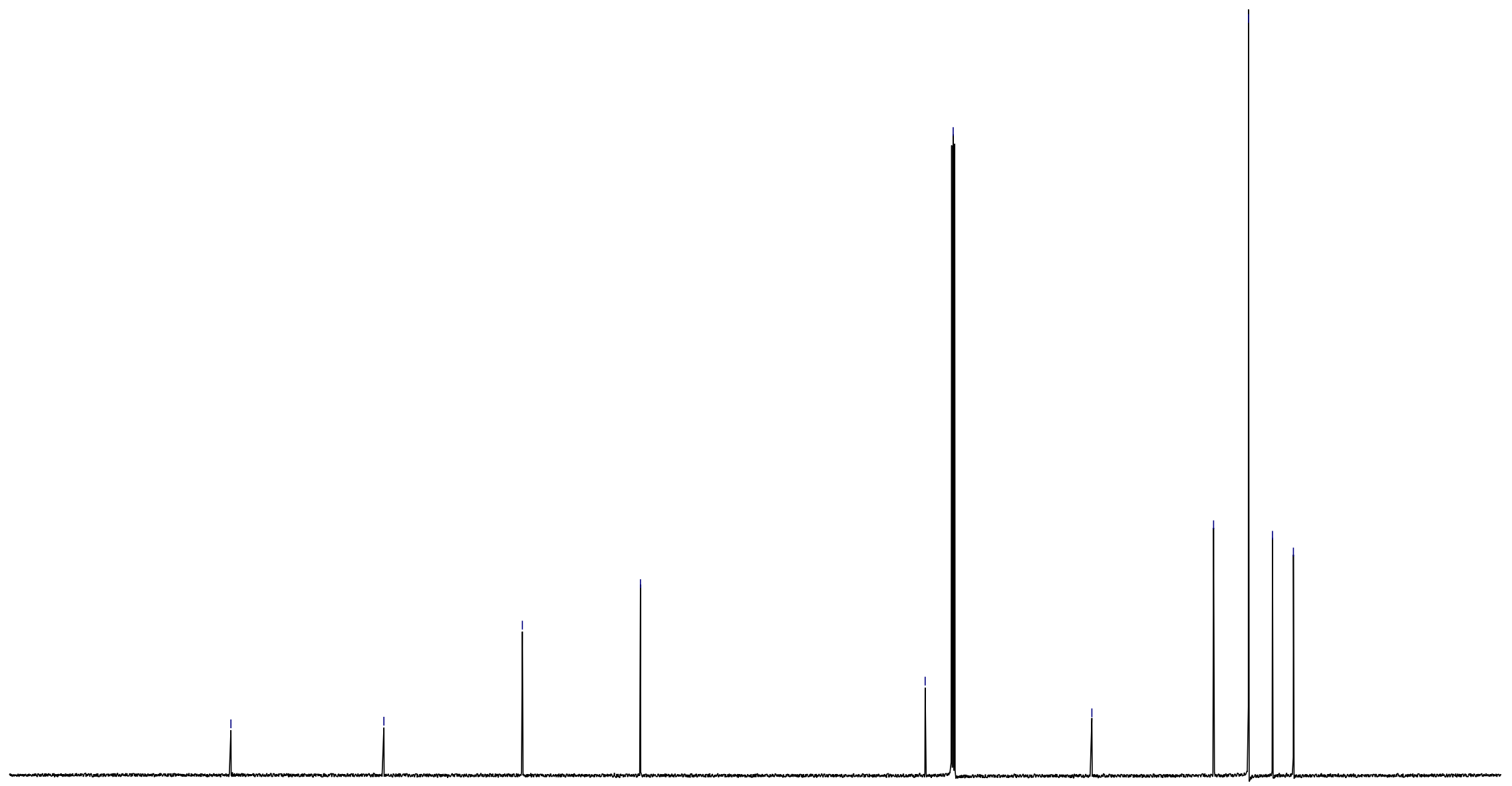




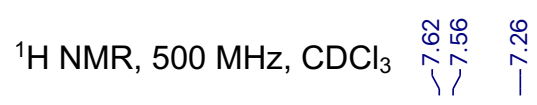

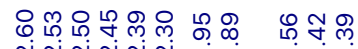

NiाT

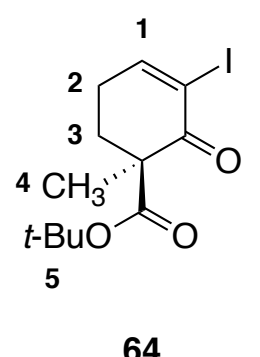

and

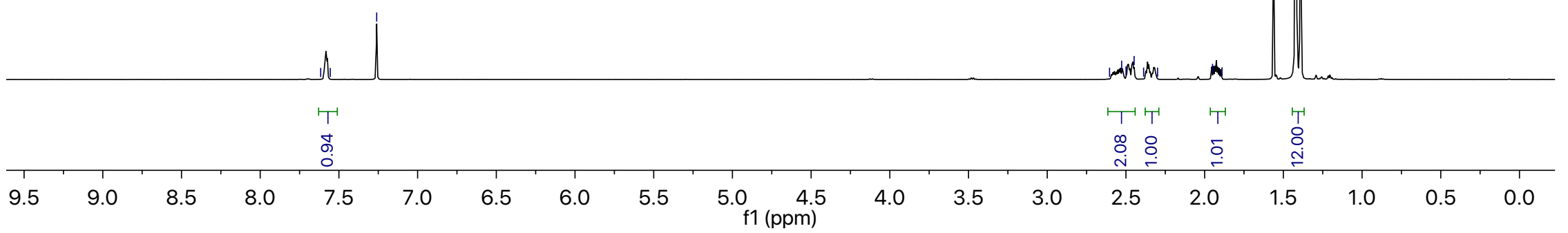


${ }^{13} \mathrm{C}$ NMR, $125 \mathrm{MHz}, \mathrm{CDCl}_{3}$

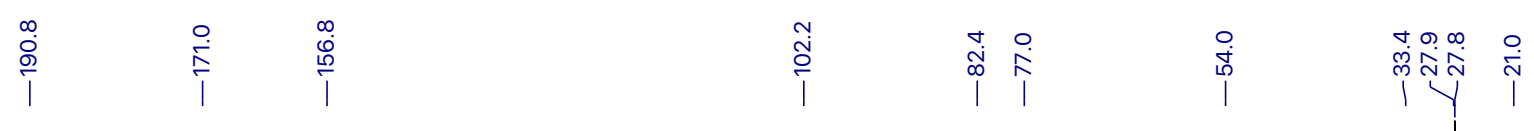

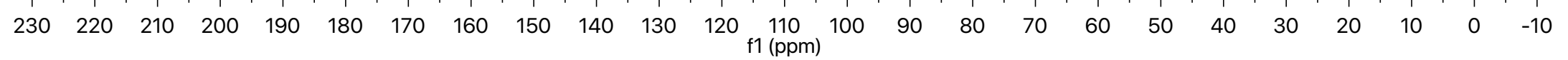


${ }^{1} \mathrm{H} \mathrm{NMR}, 600 \mathrm{MHz}, \mathrm{CDCl}_{3} \quad \stackrel{\stackrel{0}{\pi}}{i}$

ํำ

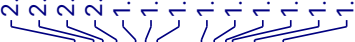

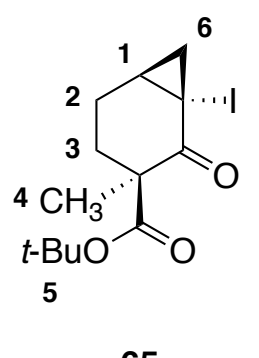

65

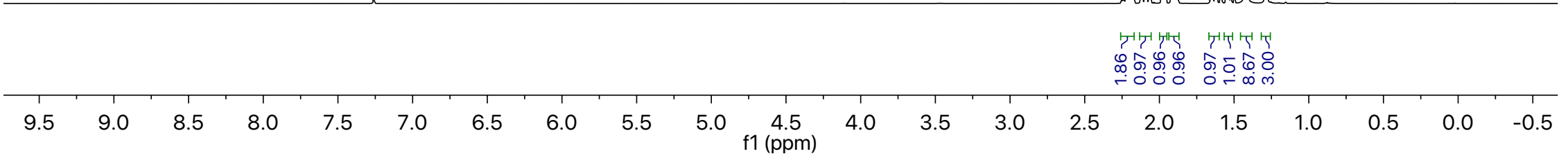


${ }^{13} \mathrm{C}$ NMR, $150 \mathrm{MHz}, \mathrm{CDCl}_{3}$ 


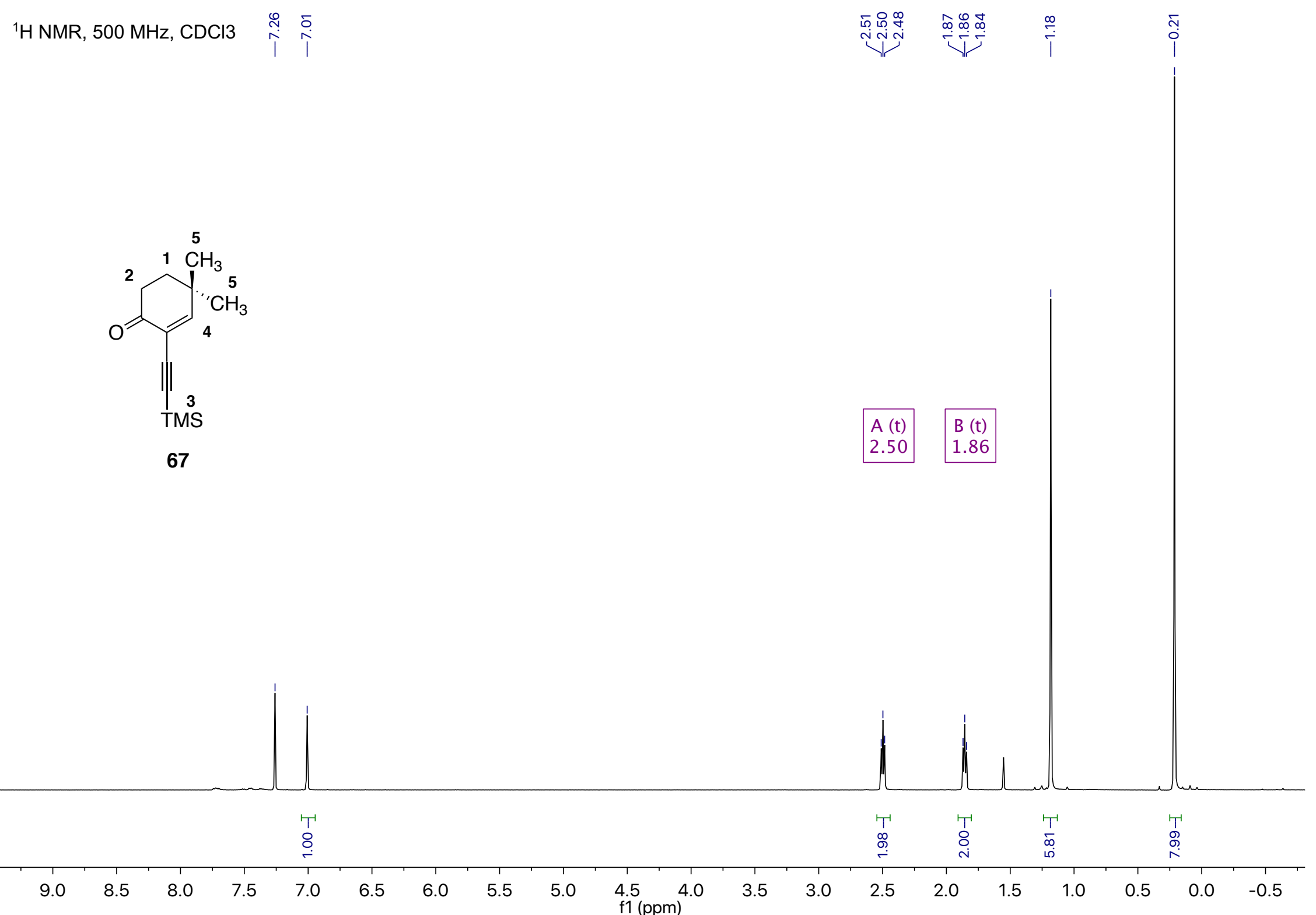


${ }^{1} \mathrm{H}$ NMR, $150 \mathrm{MHz}, \mathrm{CDCl} 3$ ํํㅇ

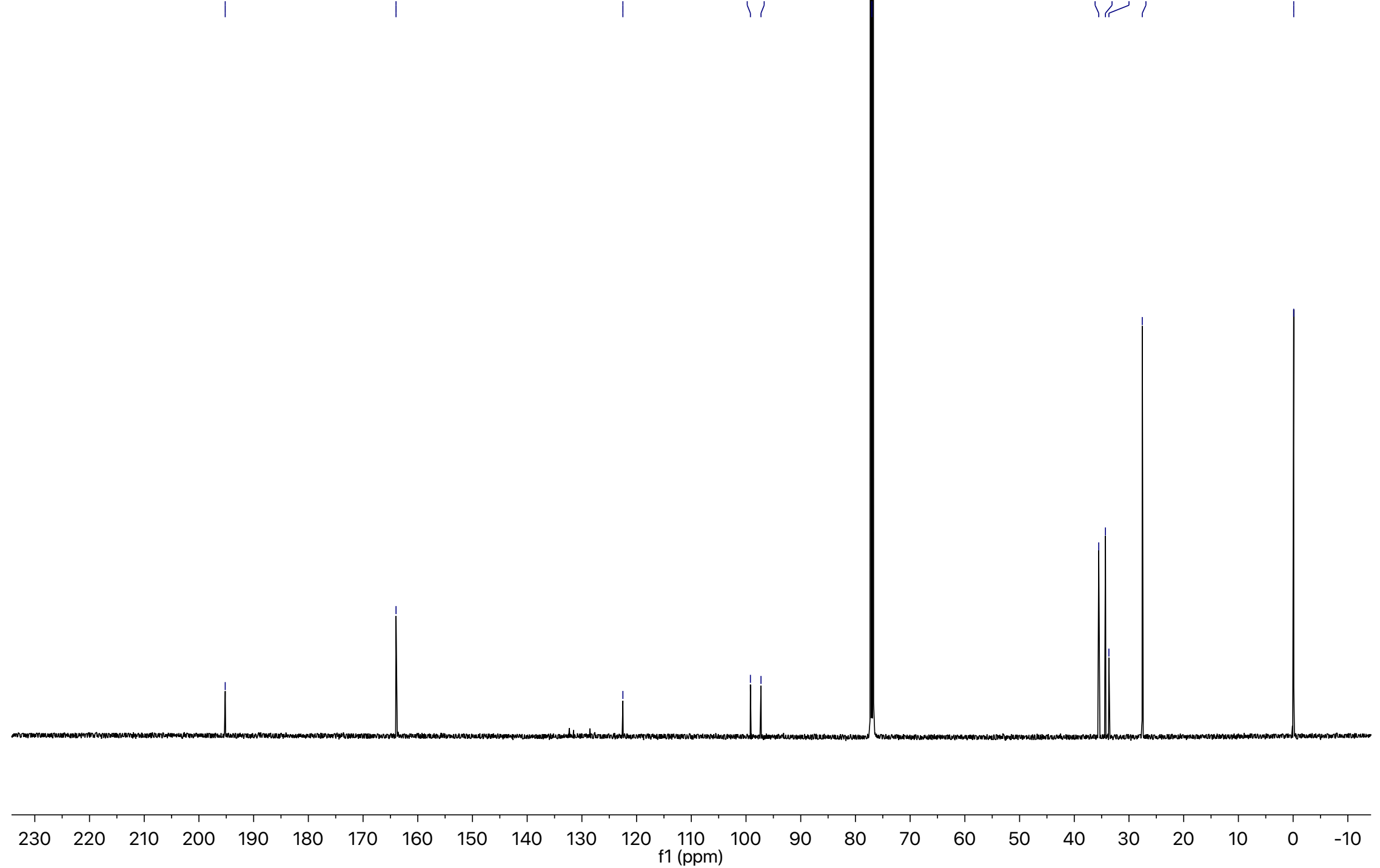




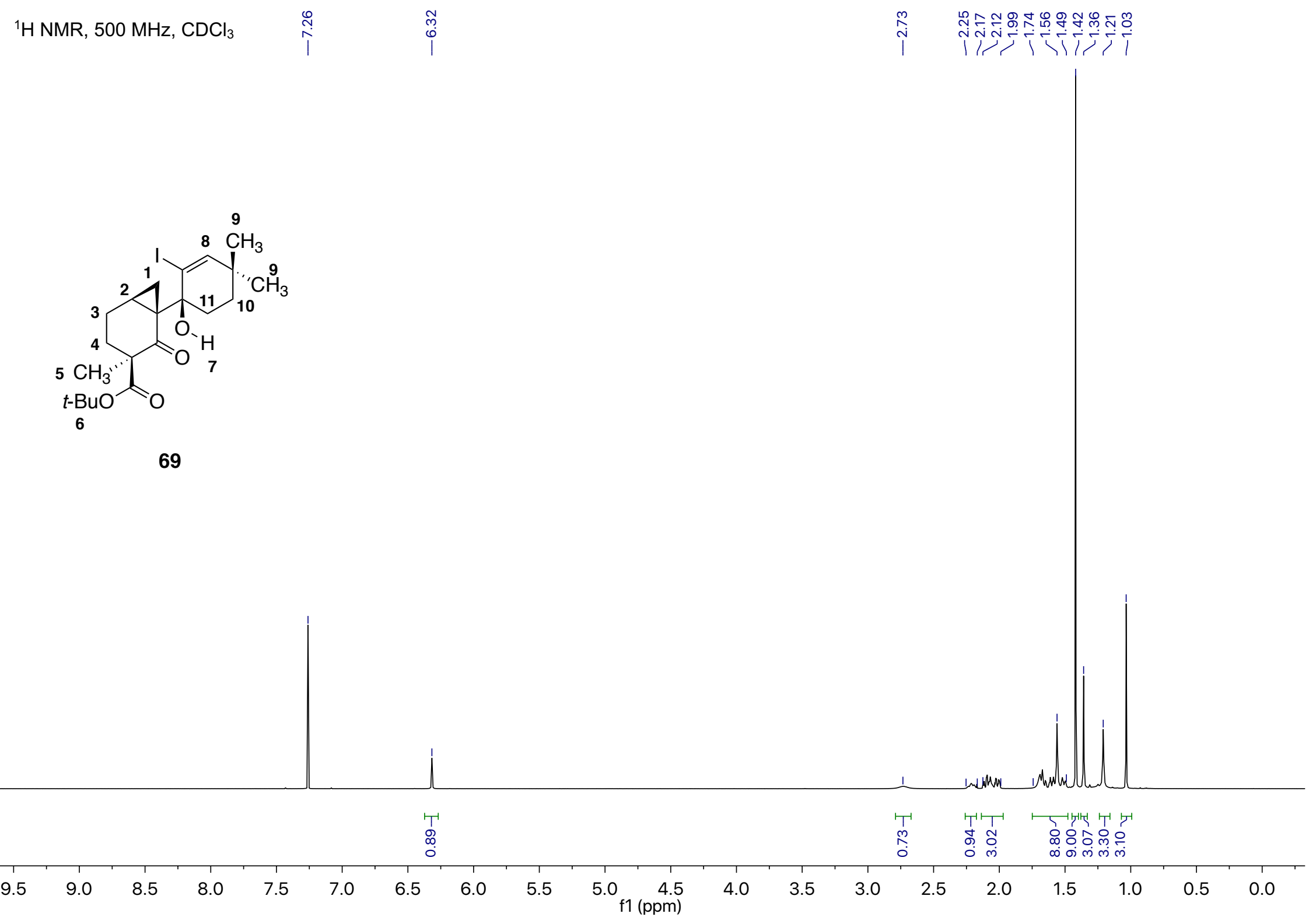



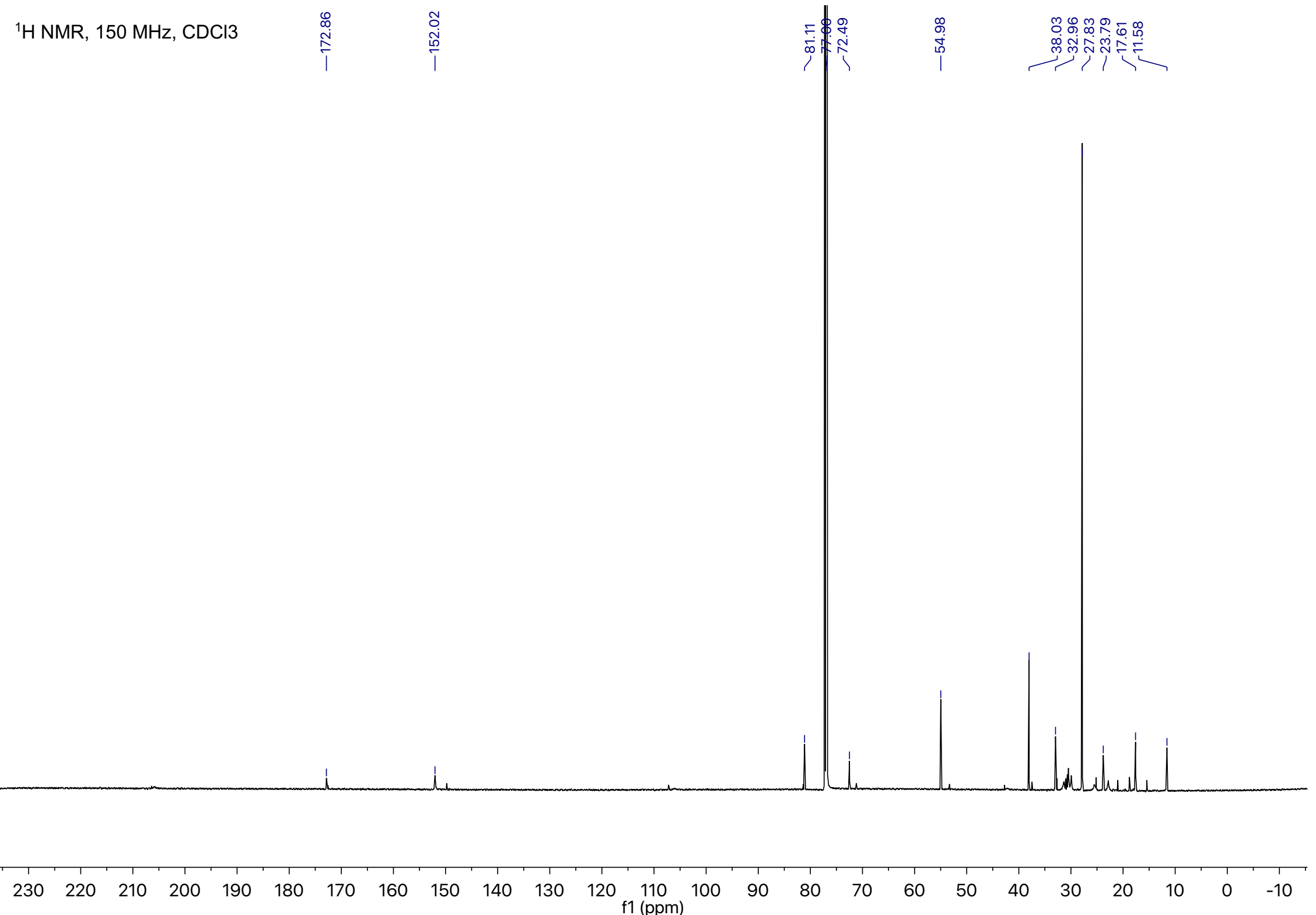


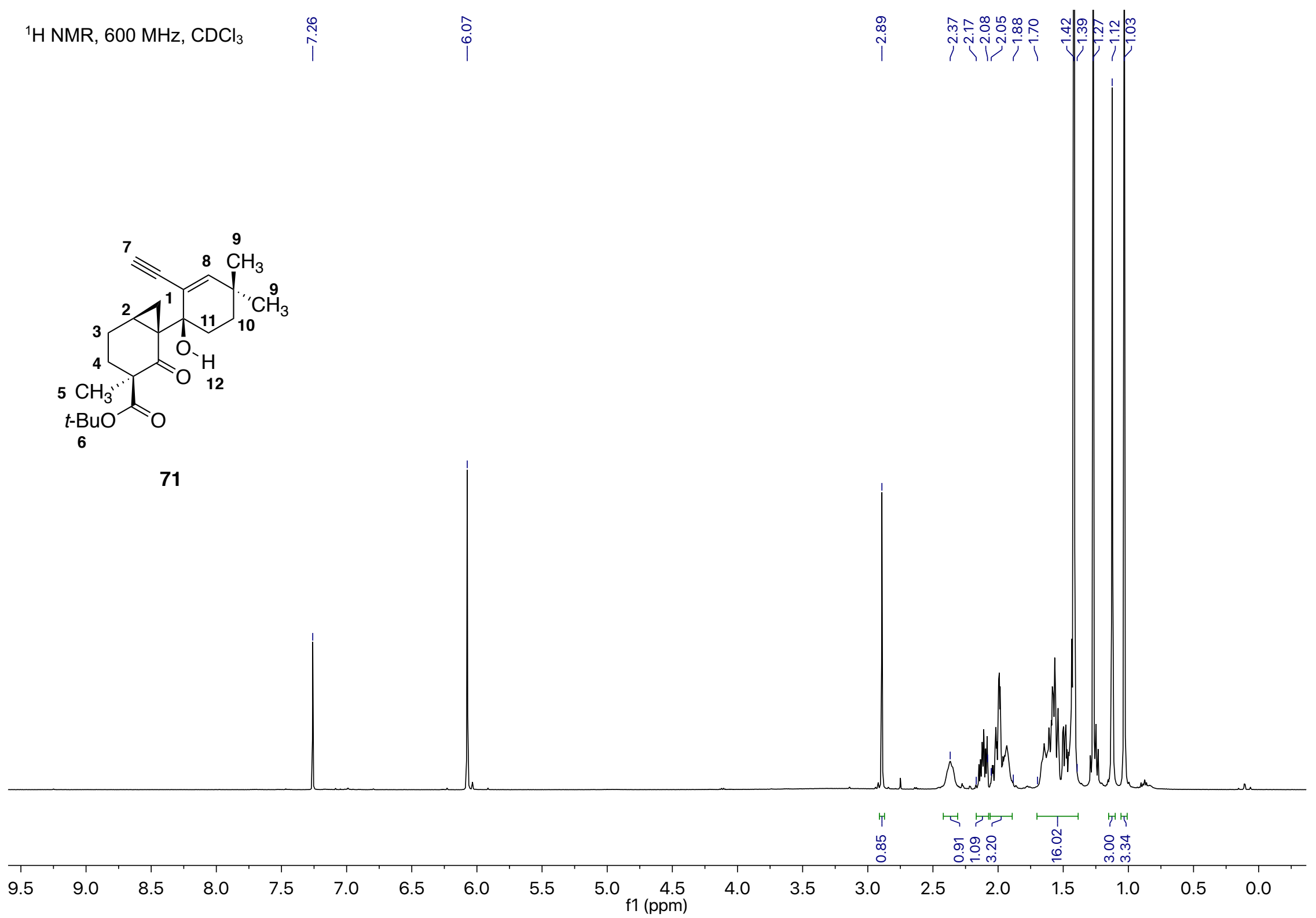


${ }^{13} \mathrm{C} \mathrm{NMR}, 125 \mathrm{MHz}, \mathrm{CDCl}_{3}$

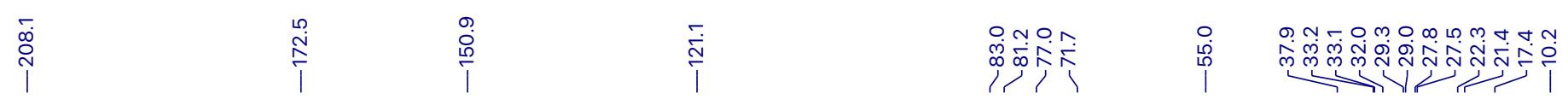

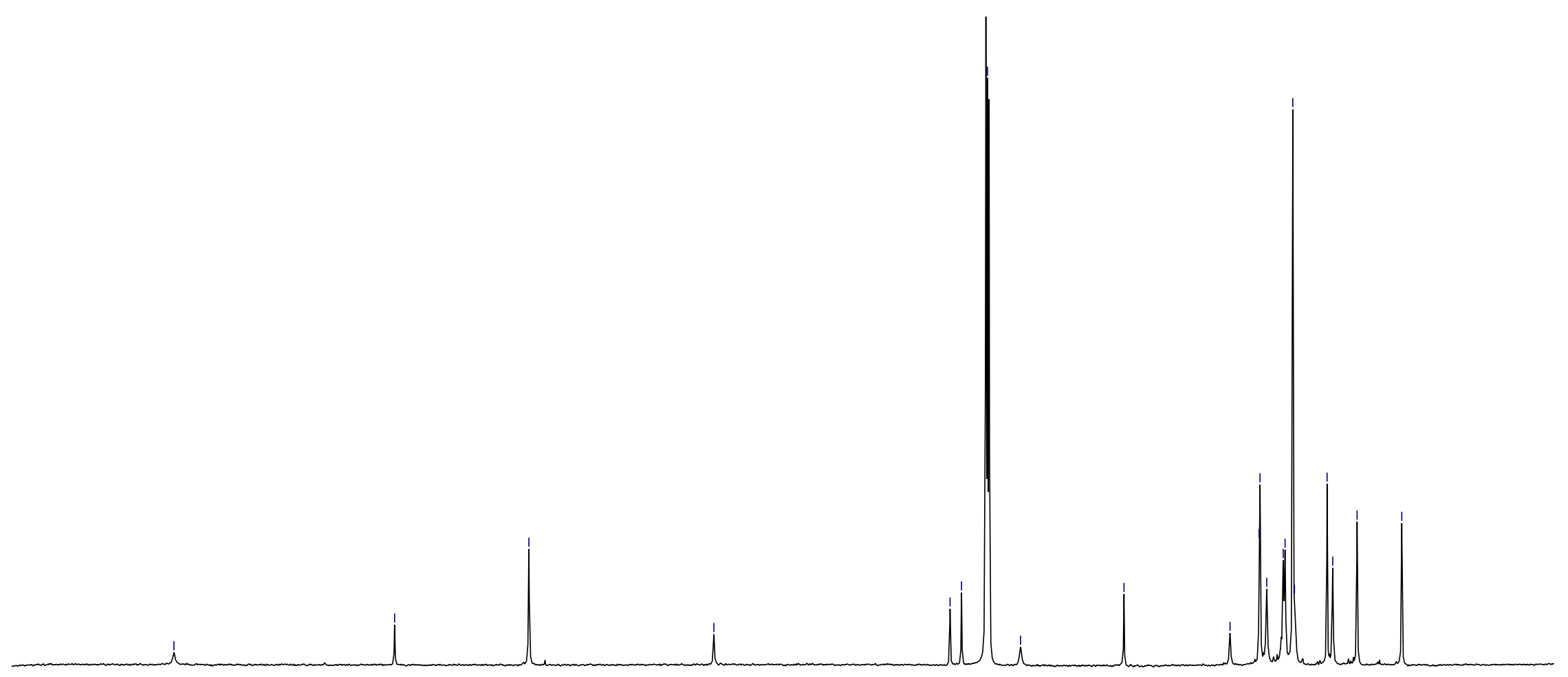

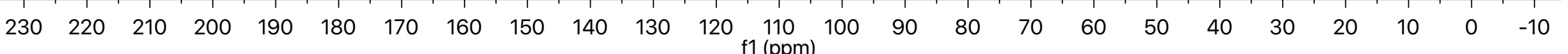




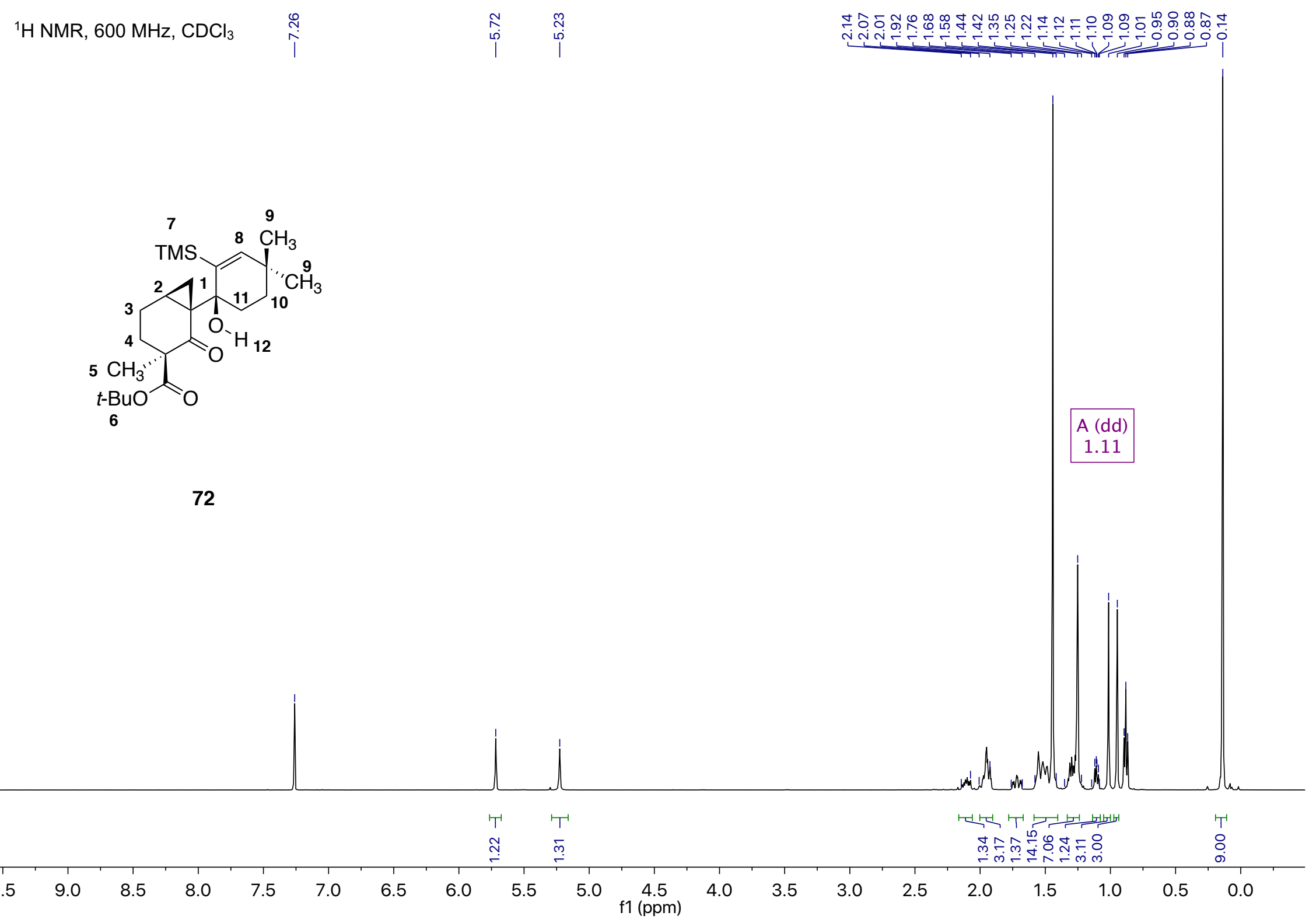


${ }^{13} \mathrm{C} \mathrm{NMR}, 150 \mathrm{MHz}, \mathrm{CDCl}_{3}$

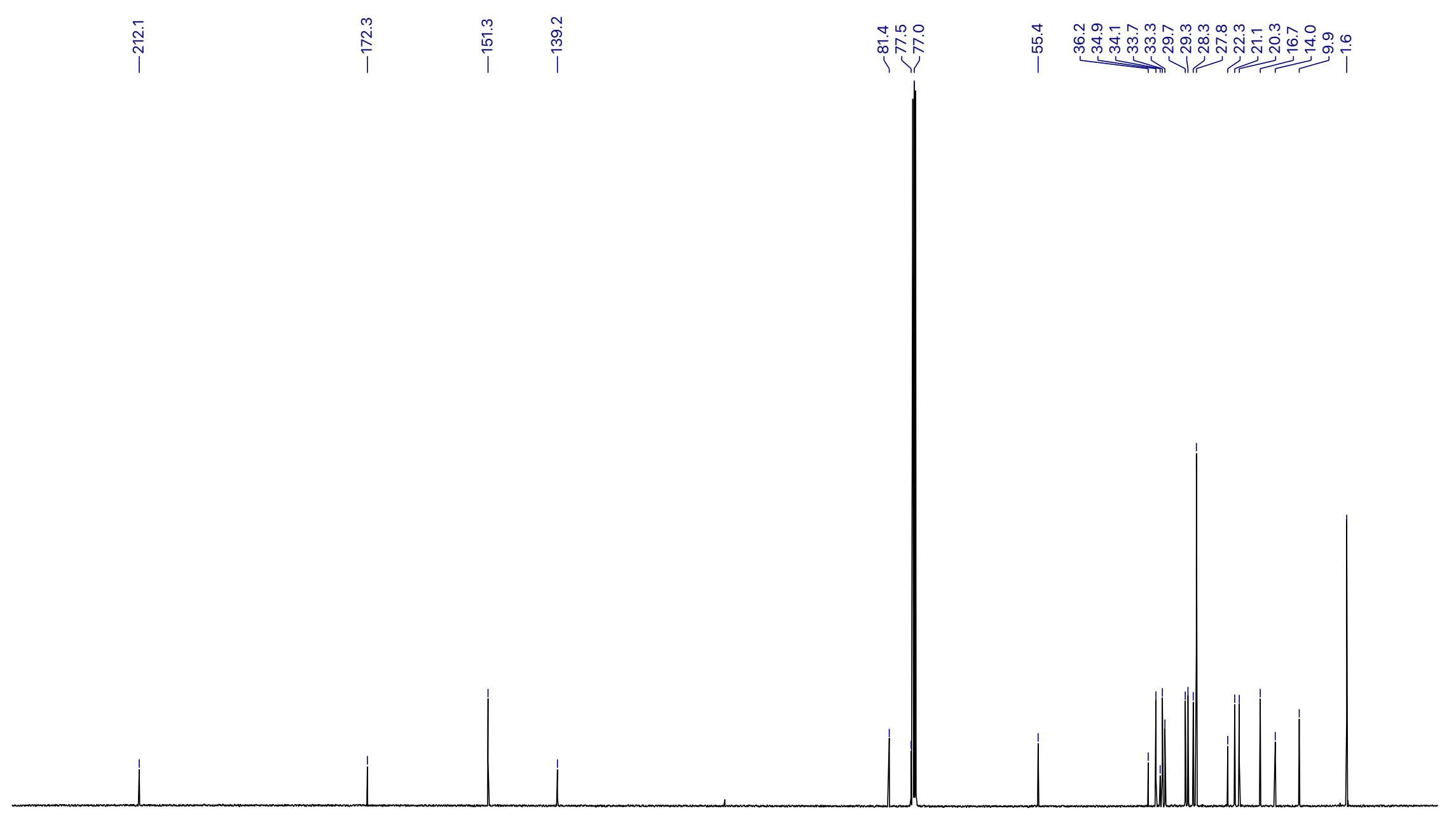

\begin{tabular}{llllllllllllllllllllllllllll}
\hline & 230 & 220 & 210 & 200 & 190 & 180 & 170 & 160 & 150 & 140 & 130 & 120 & $\begin{array}{l}110 \\
\mathrm{f} 1(\mathrm{ppm})\end{array}$ & 100 & 90 & 80 & 70 & 60 & 50 & 40 & 30 & 20 & 10 & 0 & -10
\end{tabular} 


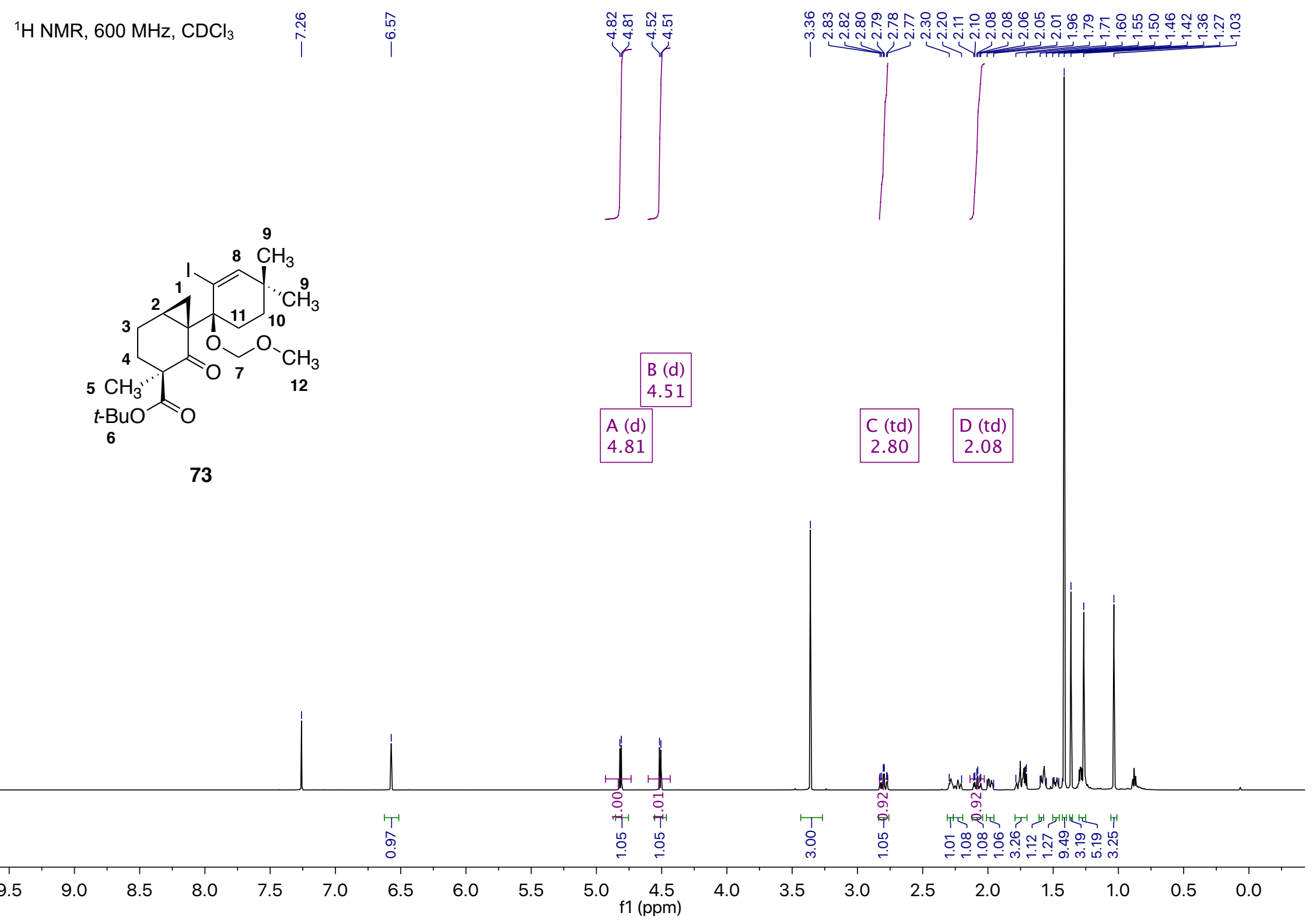




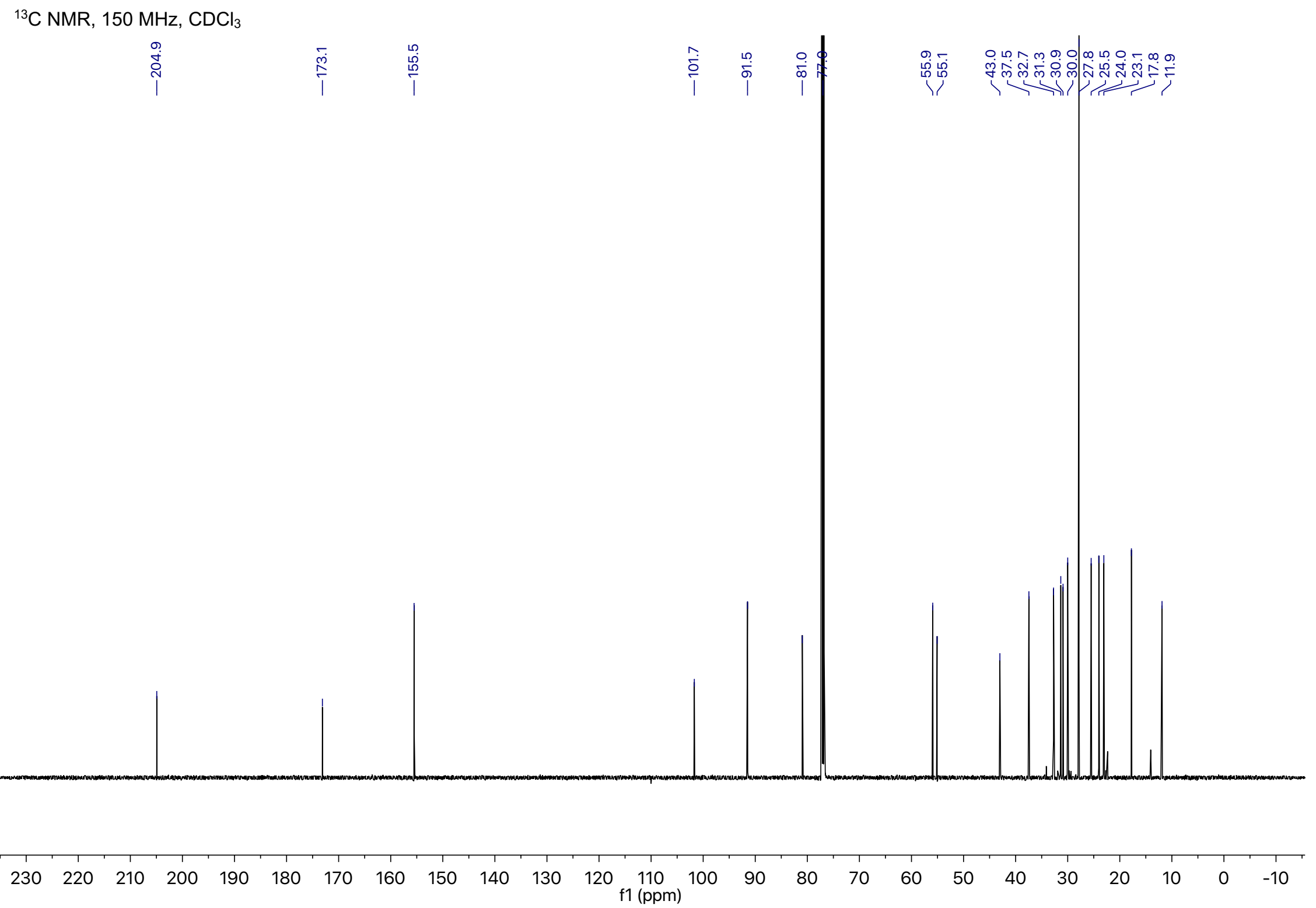




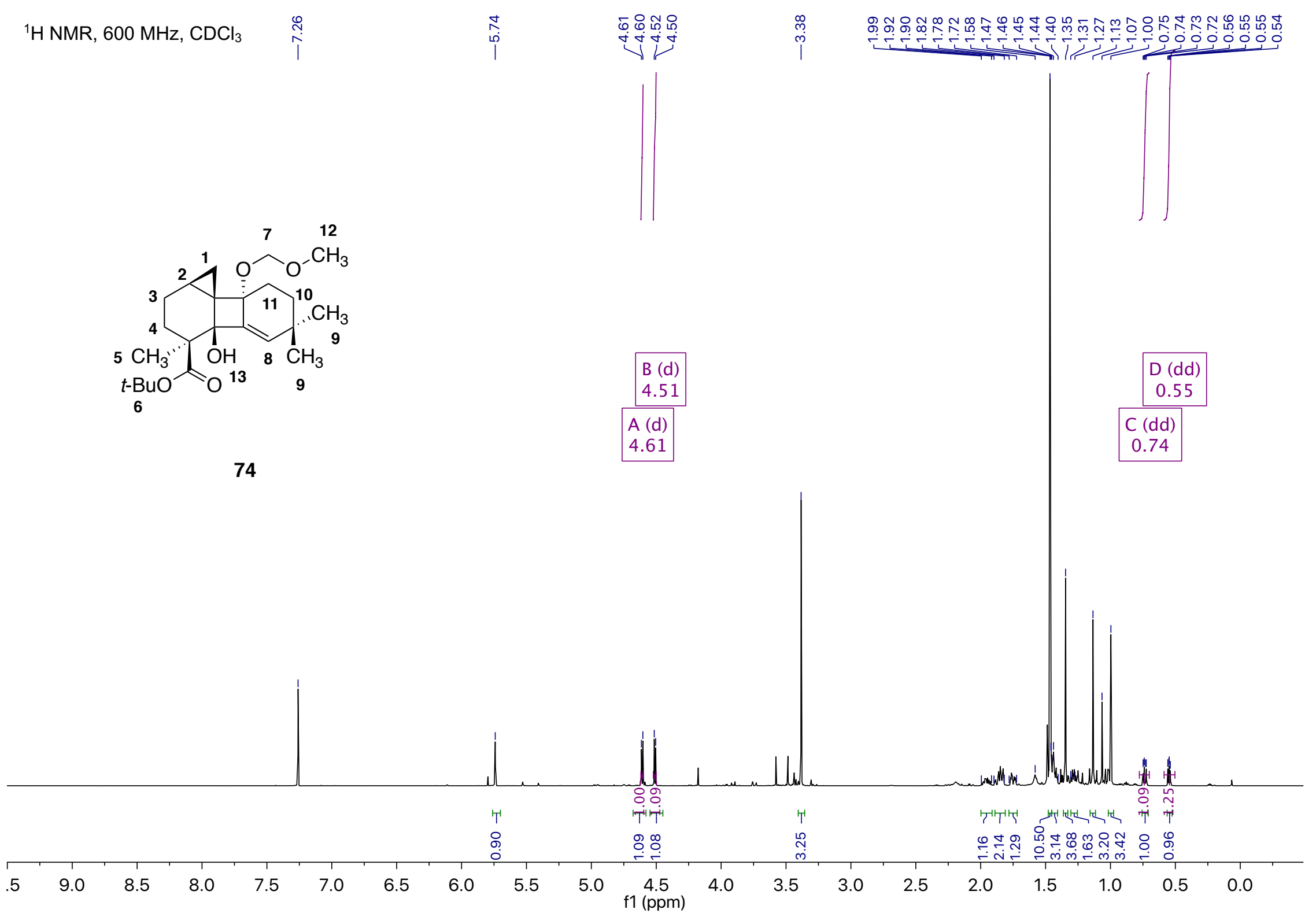




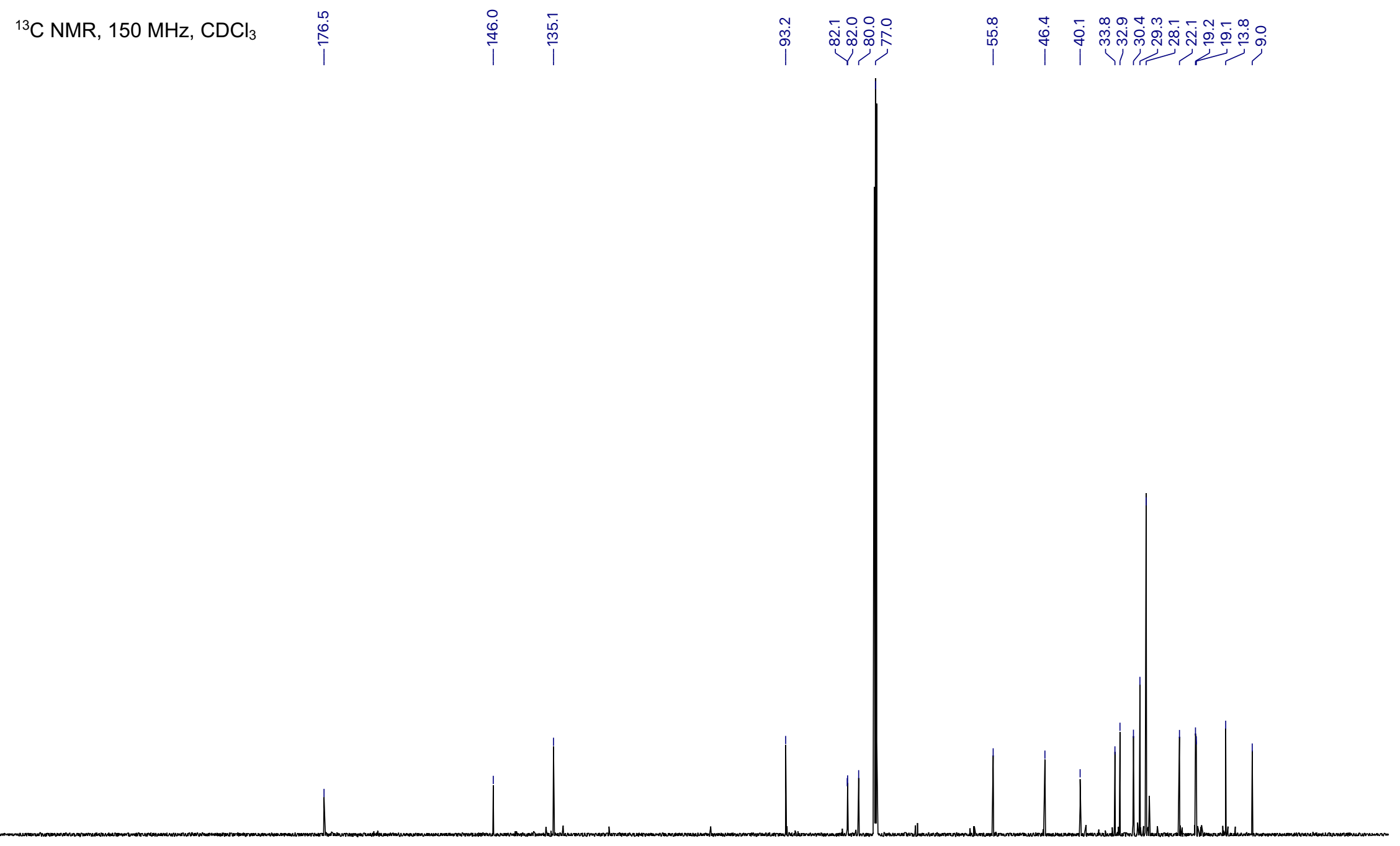

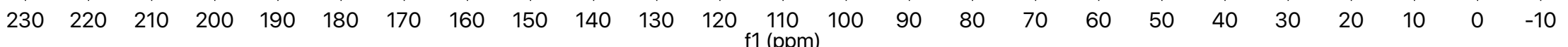




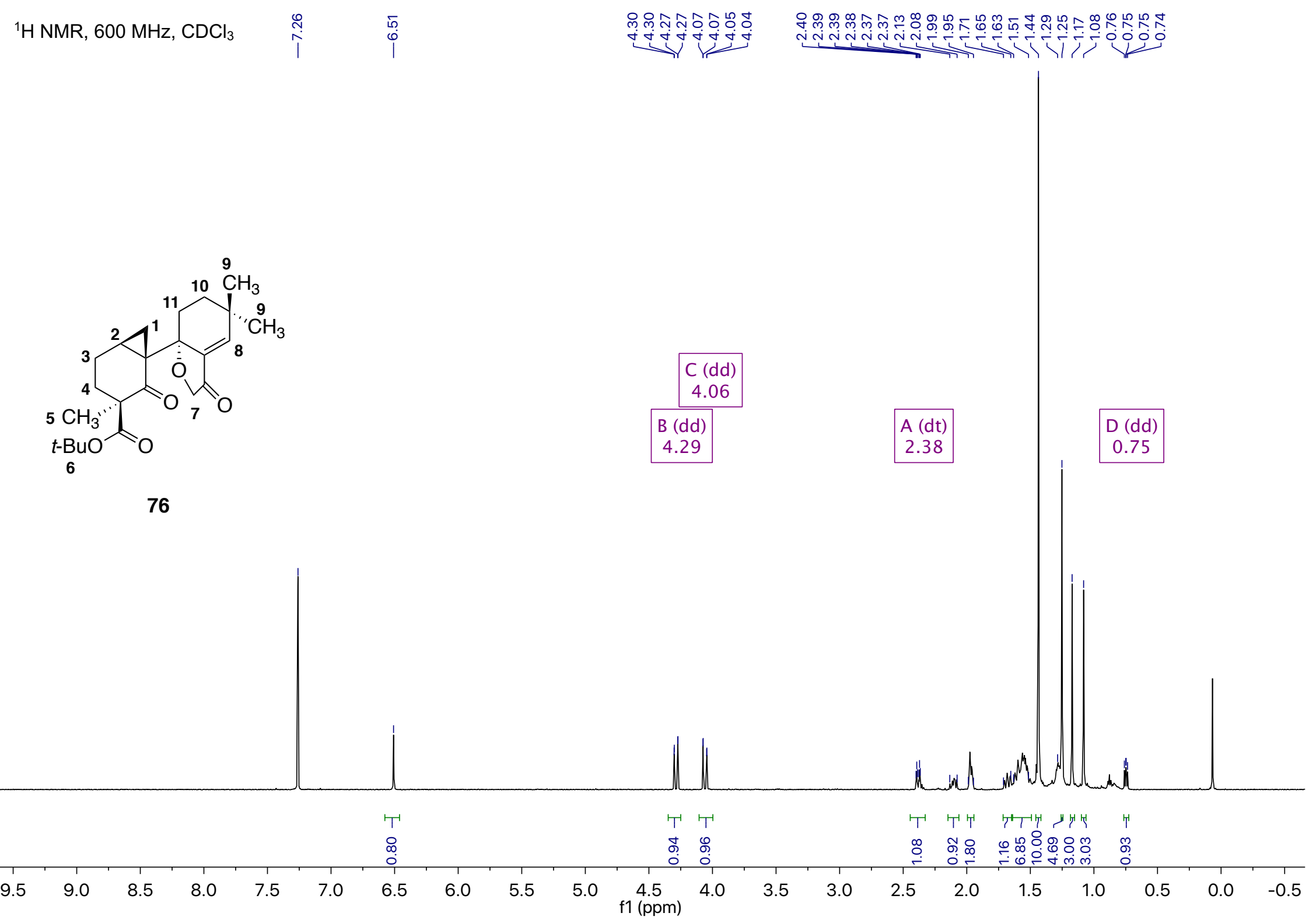




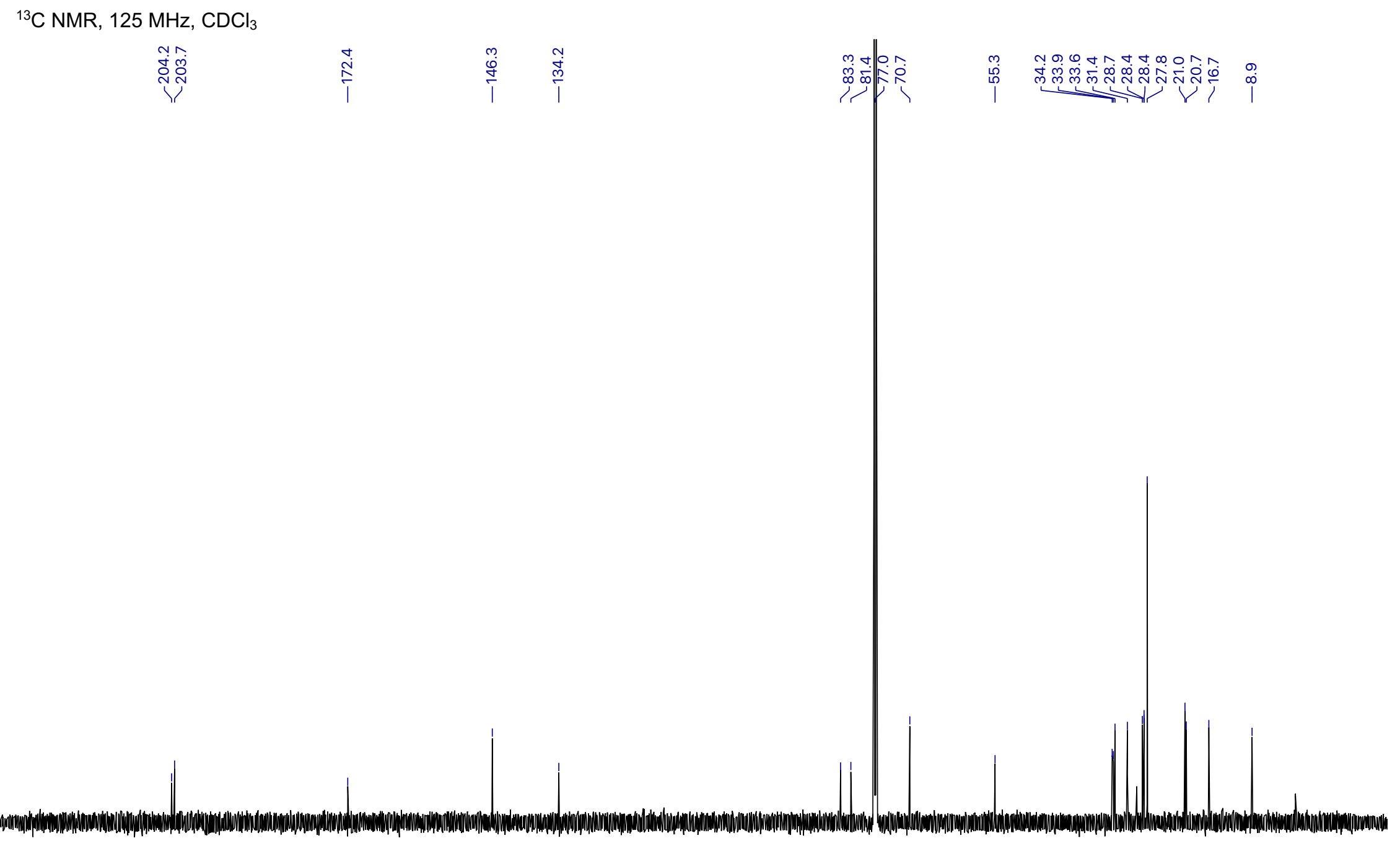

$\begin{array}{lllllllllllllllllllllllll}230 & 220 & 210 & 200 & 190 & 180 & 170 & 160 & 150 & 140 & 130 & 120 & \begin{array}{c}110 \\ \mathrm{f} 1(\mathrm{ppm})\end{array} & 100 & 90 & 80 & 70 & 60 & 50 & 40 & 30 & 20 & 10 & 0 & -10\end{array}$ 


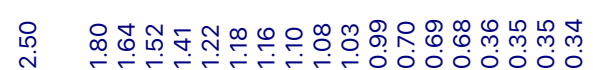

-
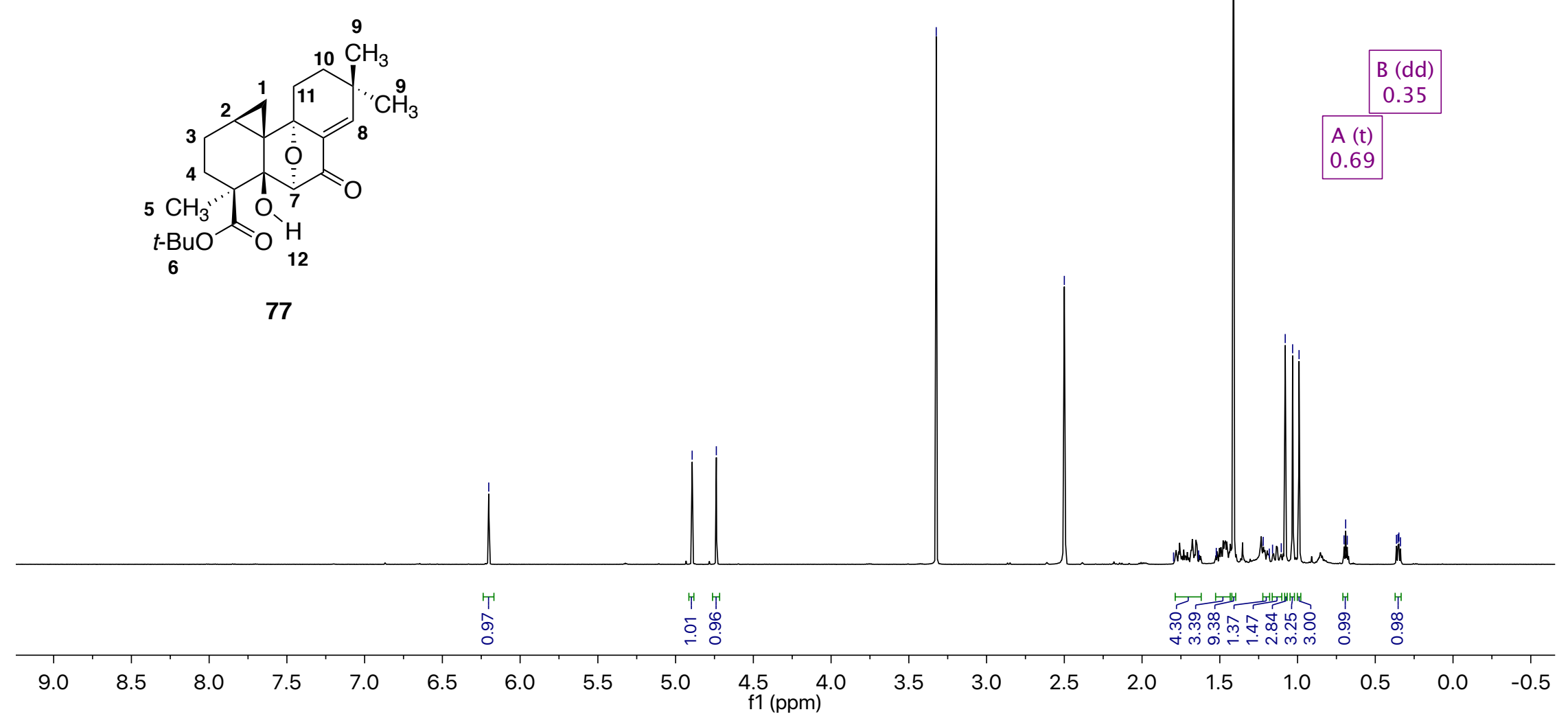


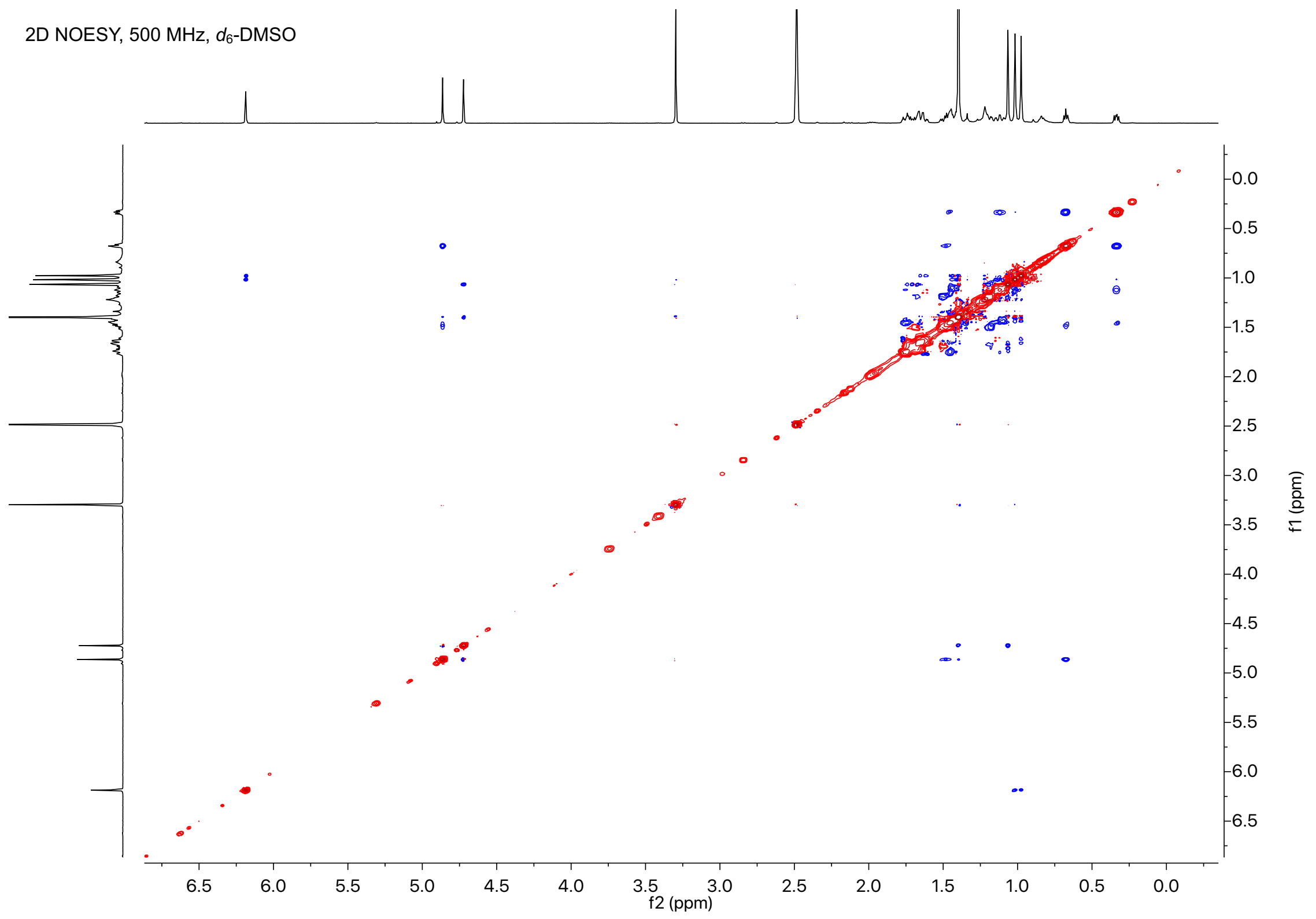


${ }^{13} \mathrm{C}$ NMR, $150 \mathrm{MHz}, d_{6}$-DMSO

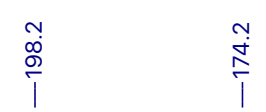

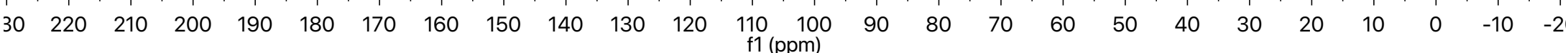


${ }^{1} \mathrm{H} \mathrm{NMR}, 500 \mathrm{MHz}, \mathrm{CDCl}_{3}$

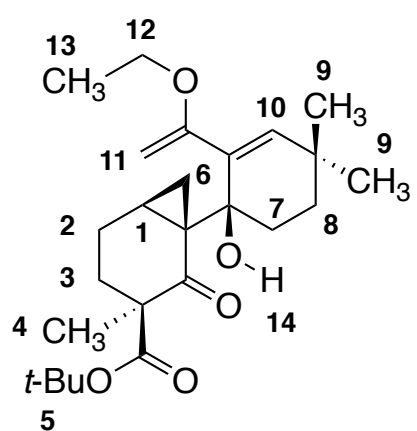

78

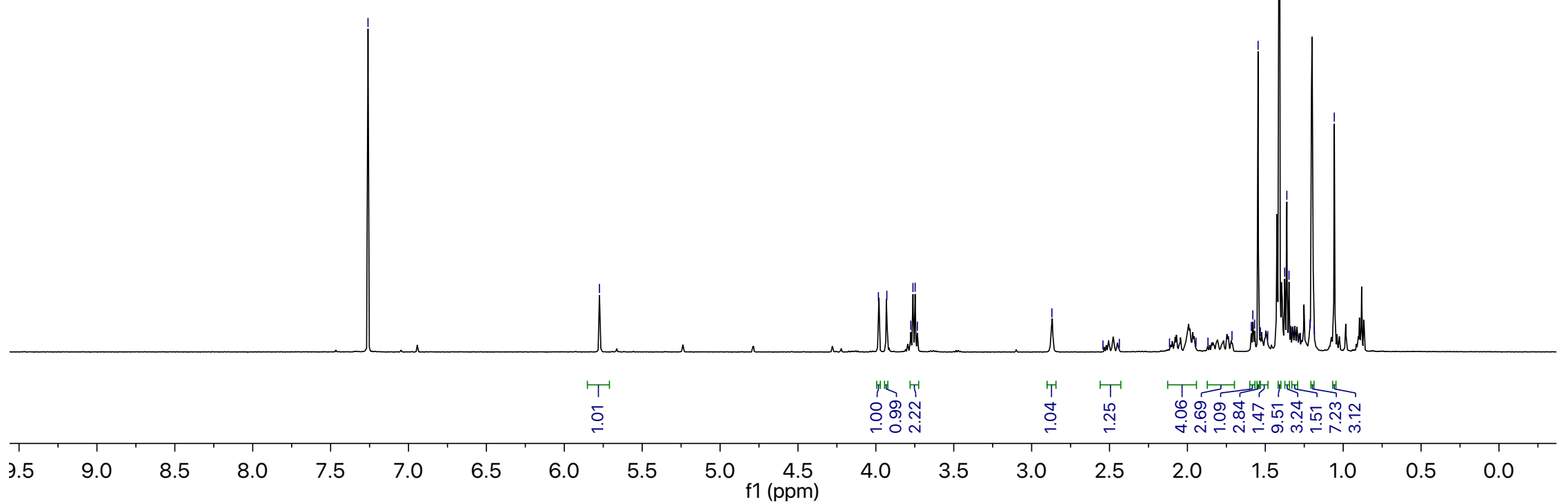


${ }^{13} \mathrm{C}$ NMR, $150 \mathrm{MHz}, \mathrm{C}_{6} \mathrm{D}_{6}$

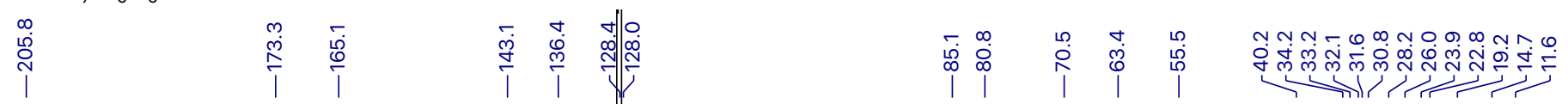

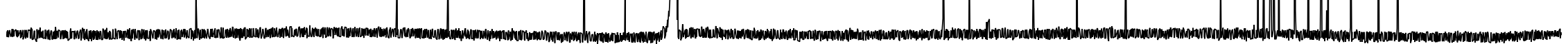

$\begin{array}{lllllllllllllllllllllllll}230 & 220 & 210 & 200 & 190 & 180 & 170 & 160 & 150 & 140 & 130 & 120 & \begin{array}{l}110 \\ \mathrm{f} 1(\mathrm{ppm})\end{array} & 100 & 90 & 80 & 70 & 60 & 50 & 40 & 30 & 20 & 10 & 0 & -10\end{array}$ 


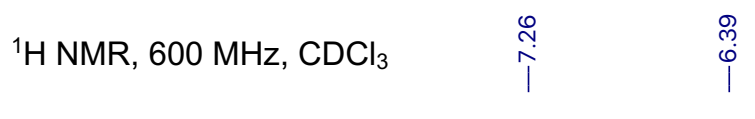

๑̊ \&

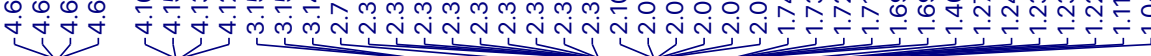

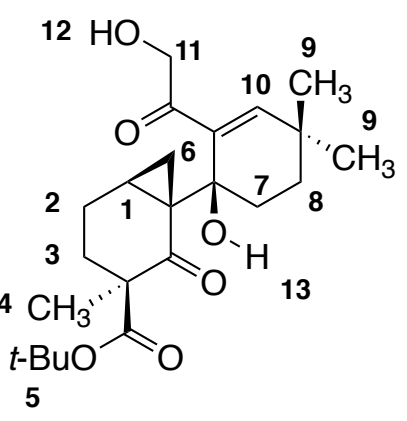

\begin{tabular}{c|c}
\hline A (dd) & B (dd) \\
4.67 & 4.14 \\
\hline
\end{tabular}

C (t)

\begin{tabular}{|c|c|c|}
\hline $\begin{array}{l}\mathrm{F}(\mathrm{tt}) \\
2.36\end{array}$ & $\begin{array}{c}\mathrm{E}(\mathrm{td}) \\
1.71\end{array}$ & \\
\hline $\begin{array}{c}\mathrm{D}(\mathrm{td}) \\
2.07\end{array}$ & & \begin{tabular}{|c}
$G(d d)$ \\
1.23
\end{tabular} \\
\hline
\end{tabular}

79

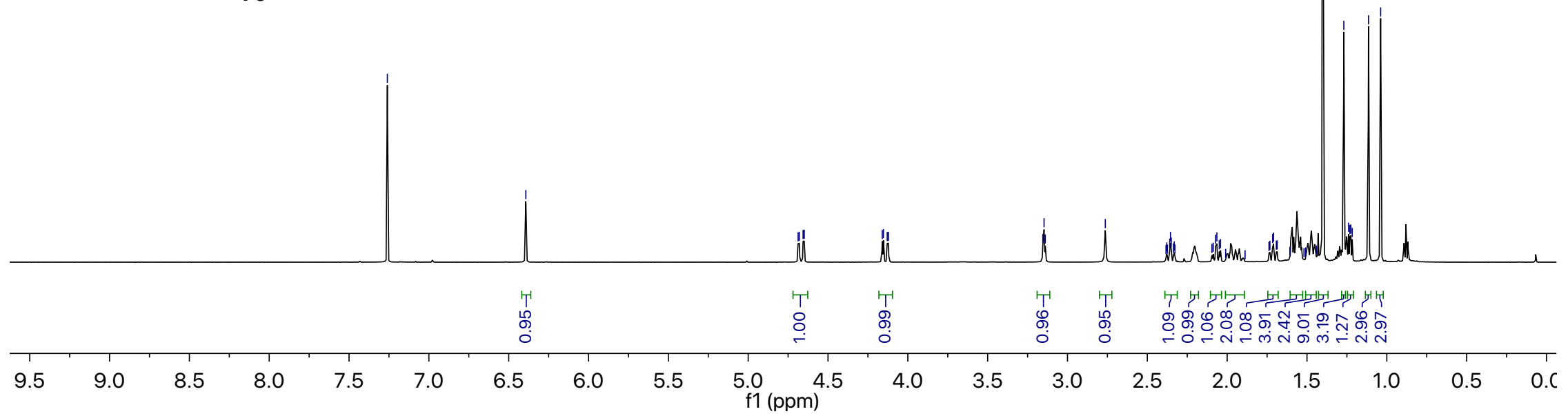


${ }^{13} \mathrm{C} \mathrm{NMR}, 150 \mathrm{MHz}, \mathrm{CDCl}_{3}$

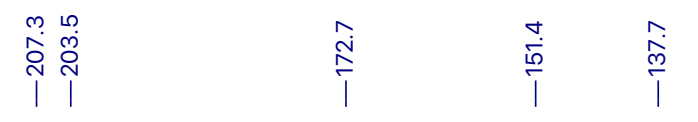

竞步

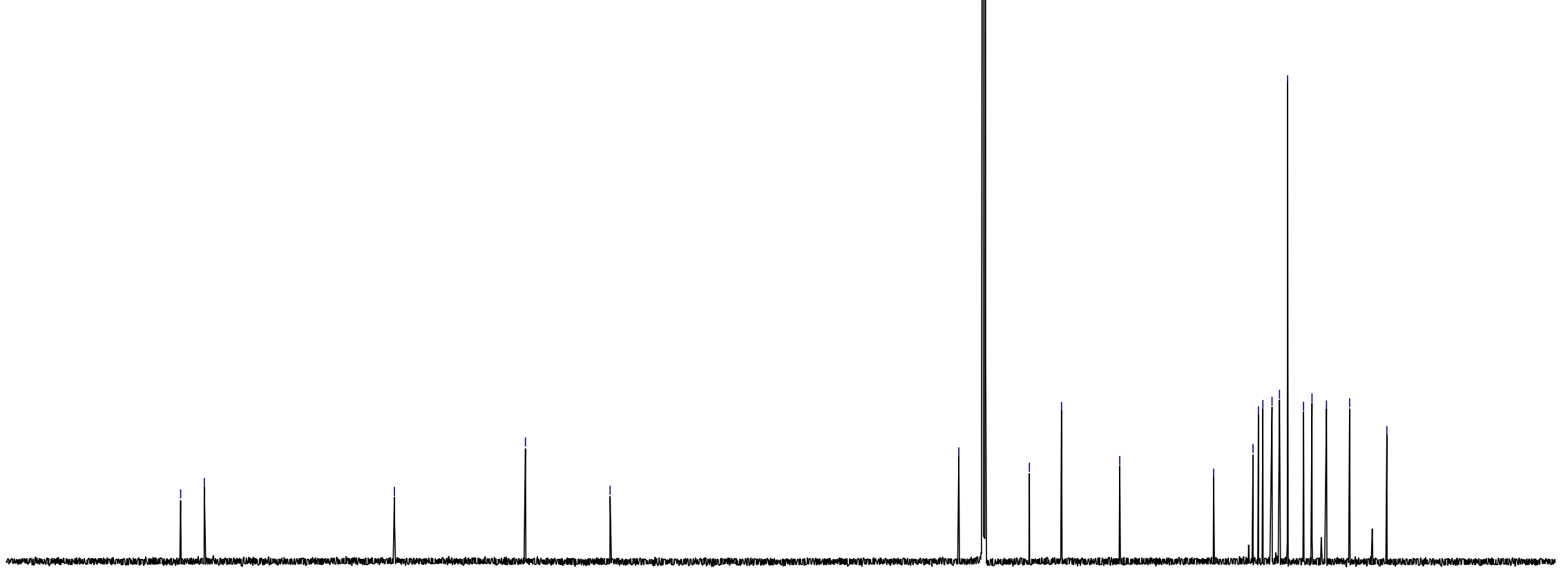

$\begin{array}{lllllllllllllllllllllllll}230 & 220 & 210 & 200 & 190 & 180 & 170 & 160 & 150 & 140 & 130 & 120 & \begin{array}{l}110 \\ \mathrm{f} 1(\mathrm{ppm})\end{array} & 100 & 90 & 80 & 70 & 60 & 50 & 40 & 30 & 20 & 10 & 0 & -10\end{array}$ 
${ }^{1} \mathrm{H} \mathrm{NMR}, 600 \mathrm{MHz}, \mathrm{CDCl}_{3}$

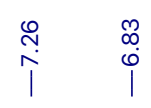

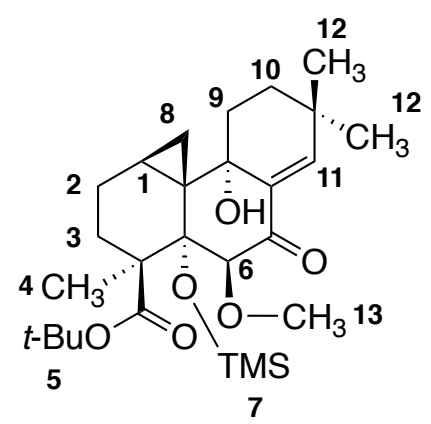

85 
${ }^{13} \mathrm{C} \mathrm{NMR}, 150 \mathrm{MHz}, \mathrm{CDCl}_{3}$

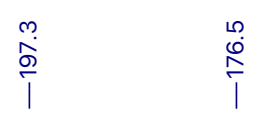

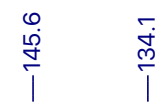

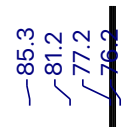

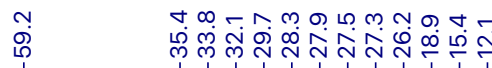

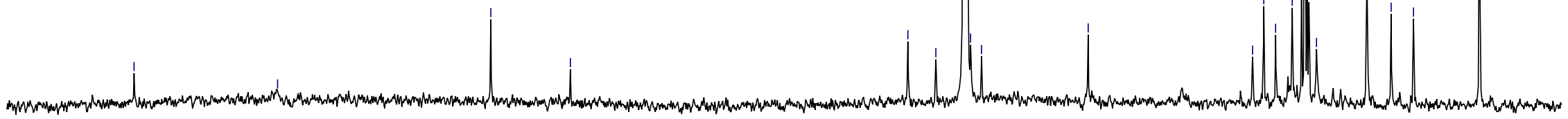

$\begin{array}{llllllllllll}210 & 200 & 190 & 180 & 170 & 160 & 150 & 140 & 130 & 120 & 110 & 100\end{array}$

90

80

70

60

50

40

30

20

10 


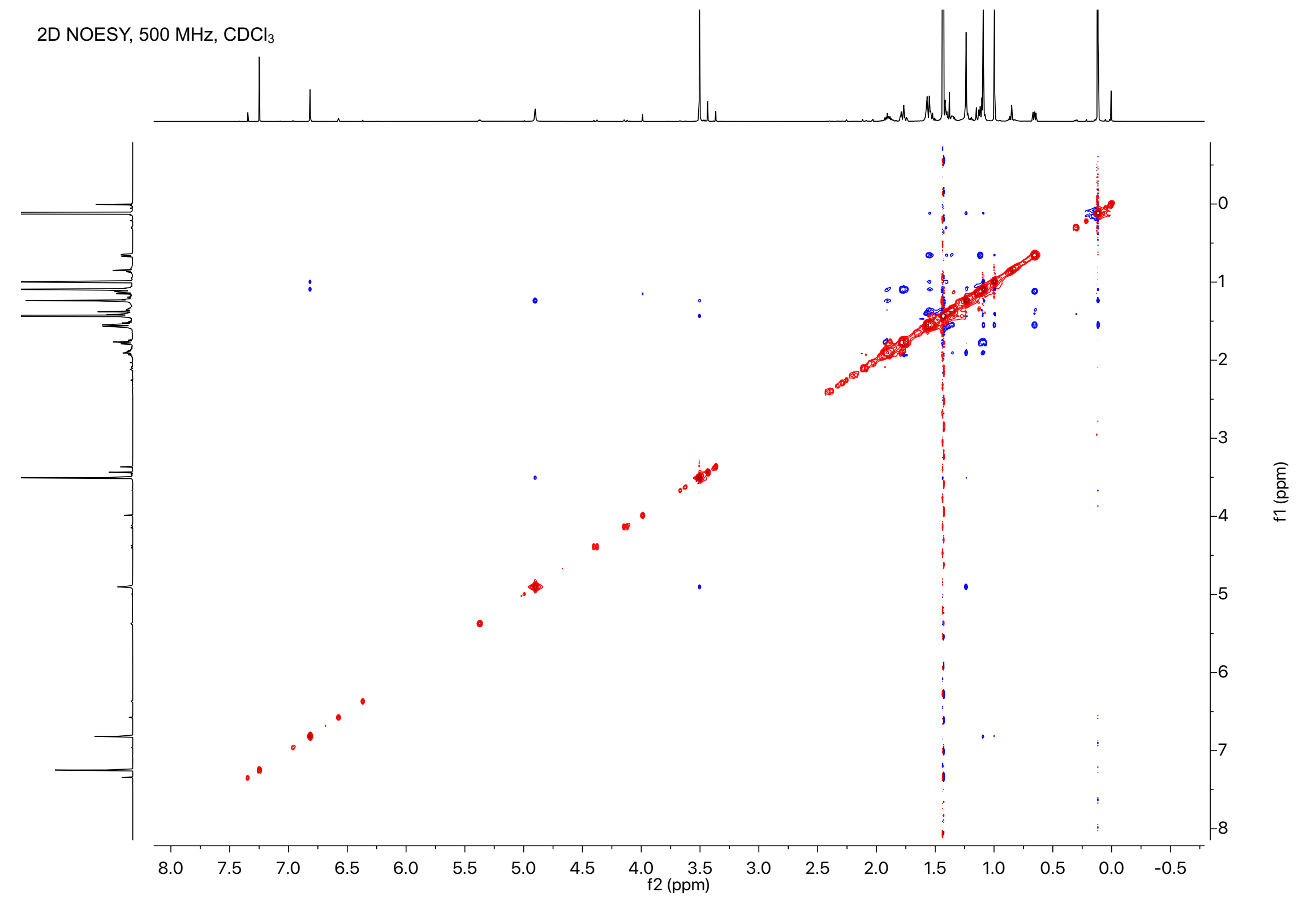


${ }^{1} \mathrm{H}$ NMR, $500 \mathrm{MHz}, \mathrm{CDCl} 3$
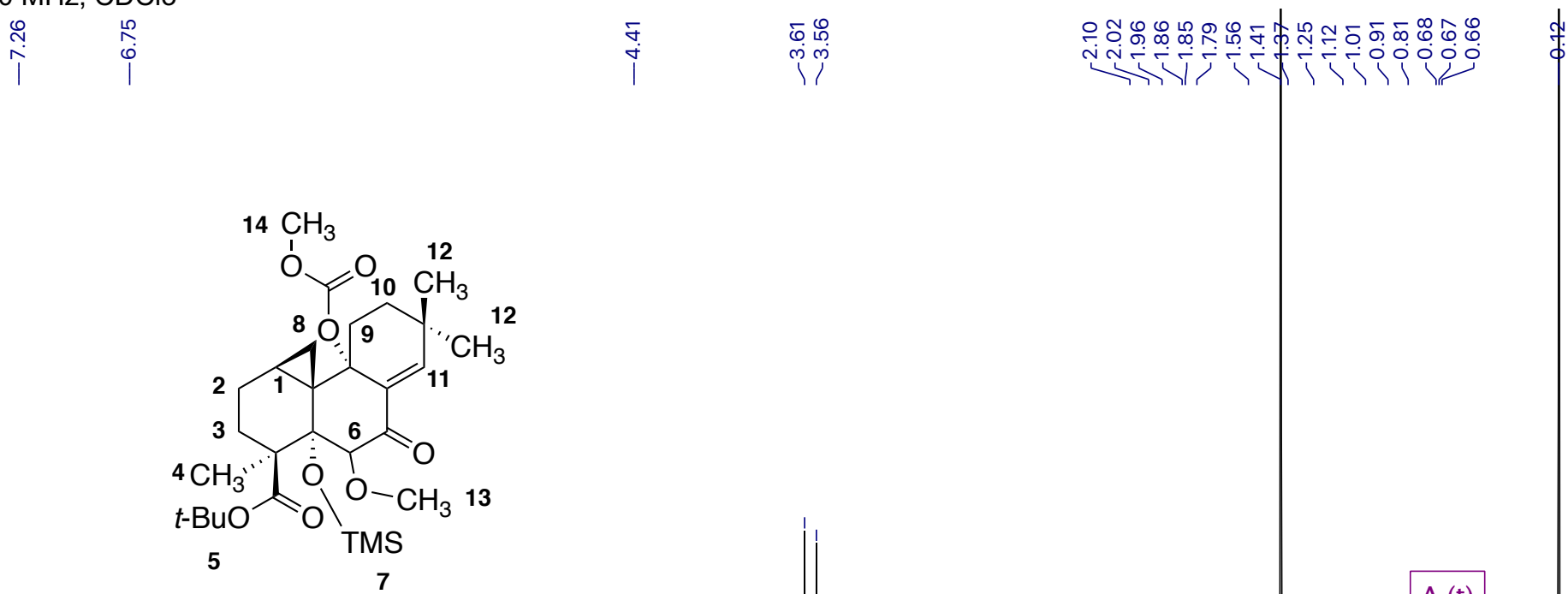

86

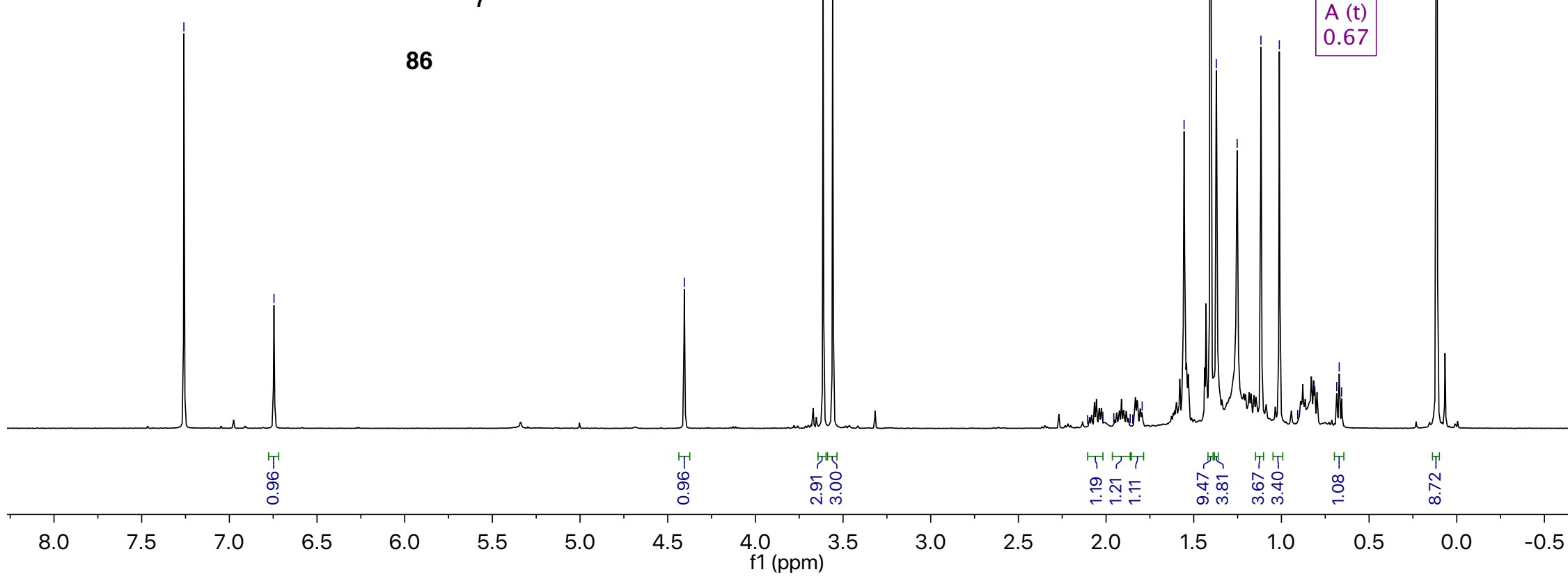


${ }^{13} \mathrm{C}$ NMR, $125 \mathrm{MHz}, \mathrm{CDCl}_{3}$

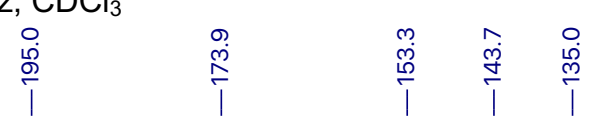

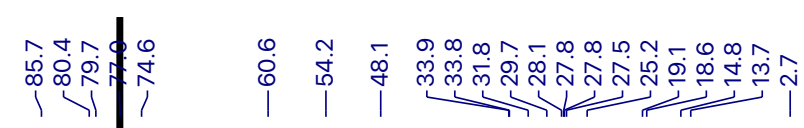

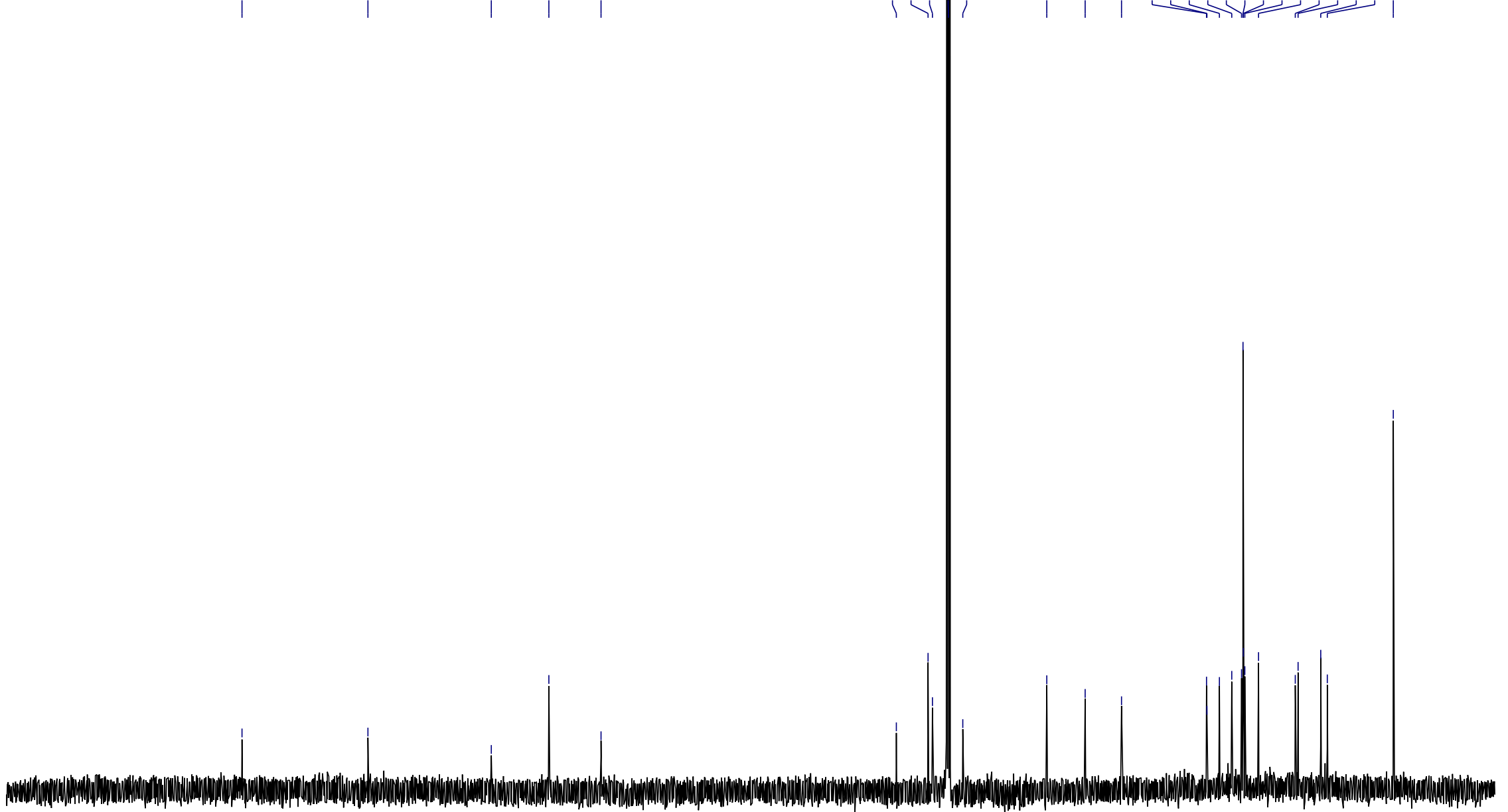

230

$\begin{array}{lllll}220 & 210 & 200 & 190 & 180\end{array}$

$180 \quad 170$

160150

140

130

$120 \quad 110$

90

80

60

50

$40 \quad 30$

20

10

$0 \quad-10$ 


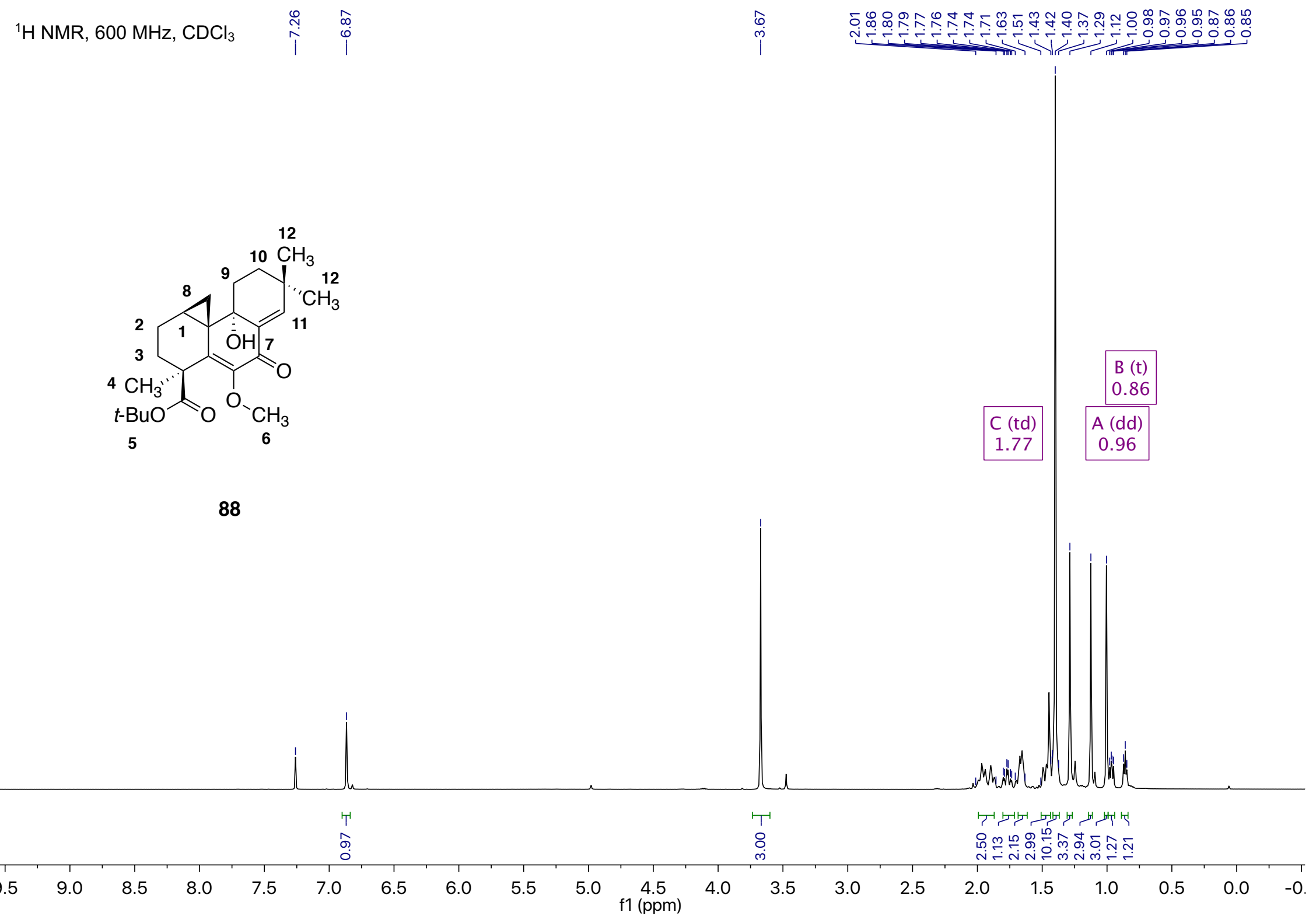




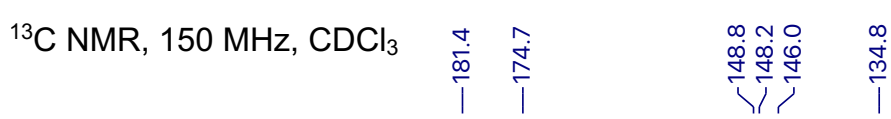

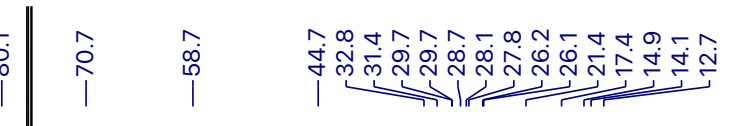

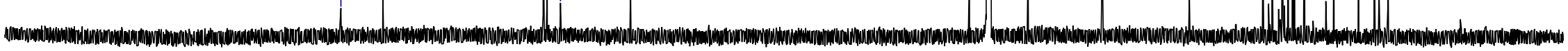

$\begin{array}{lllllllllllllllllllllllll}230 & 220 & 210 & 200 & 190 & 180 & 170 & 160 & 150 & 140 & 130 & 120 & \begin{array}{c}110 \\ \mathrm{f} 1(\mathrm{ppm})\end{array} & 100 & 90 & 80 & 70 & 60 & 50 & 40 & 30 & 20 & 10 & 0 & -10\end{array}$


${ }^{1} \mathrm{H} \mathrm{NMR}, 600 \mathrm{MHz}, \mathrm{CDCl}_{3}$

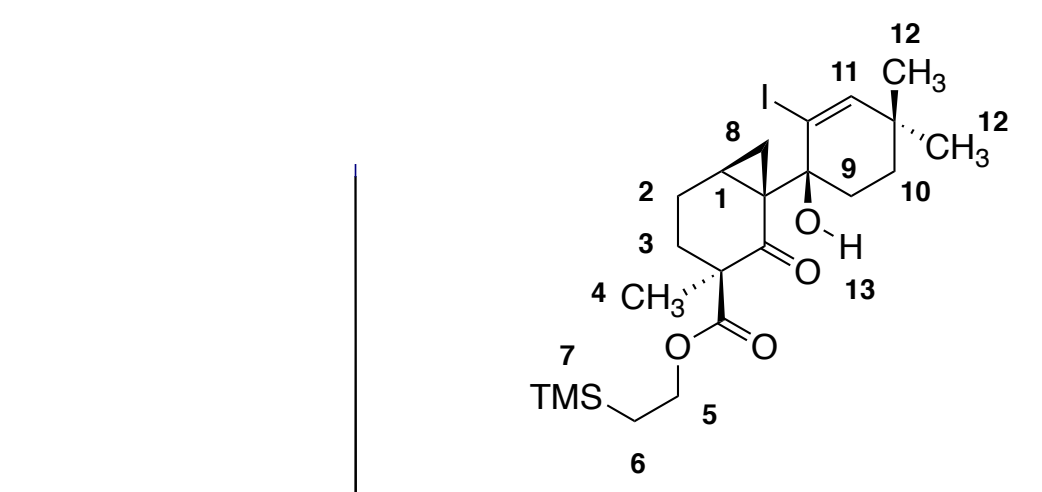

90

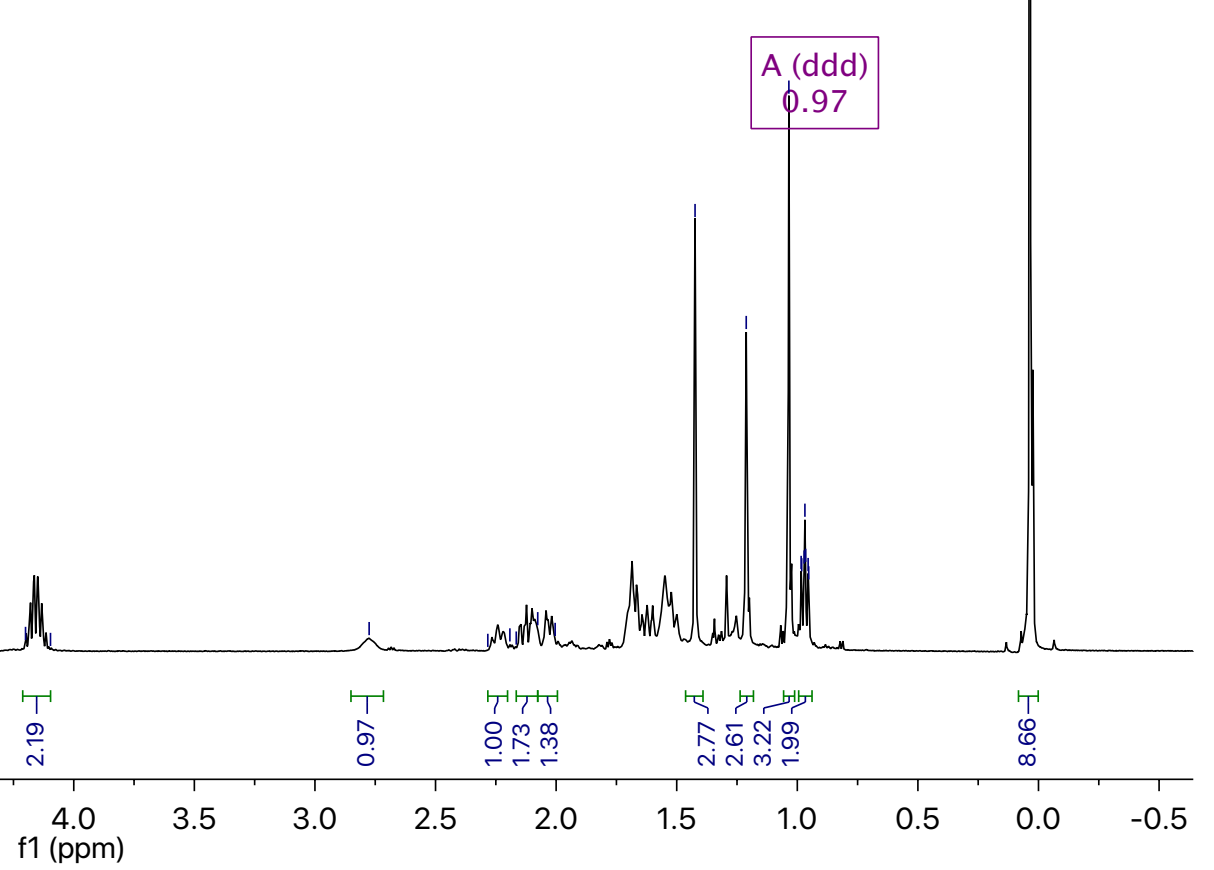


${ }^{13} \mathrm{C}$ NMR, $150 \mathrm{MHz}, \mathrm{CDCl}_{3}$

$$
\text { นัก }
$$

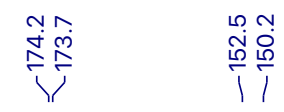

$\stackrel{\circ}{\text { 음 }}$
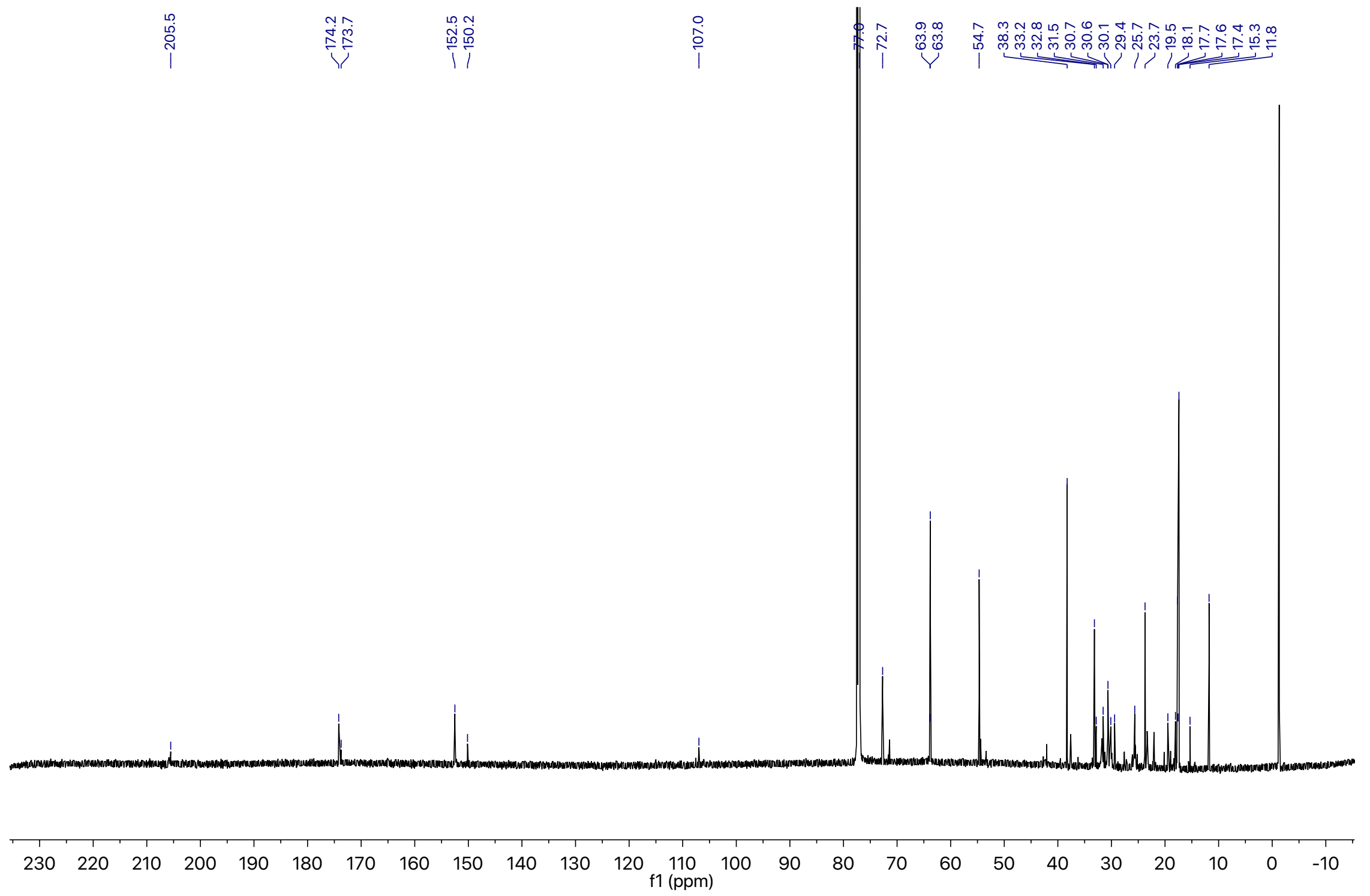
${ }^{1} \mathrm{H} \mathrm{NMR}, 500 \mathrm{MHz}, \mathrm{CDCl}_{3} \quad \stackrel{\stackrel{0}{i}}{i} \quad \stackrel{?}{0}$

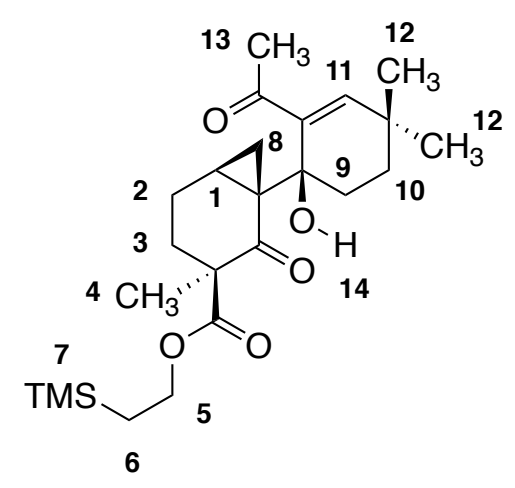

91
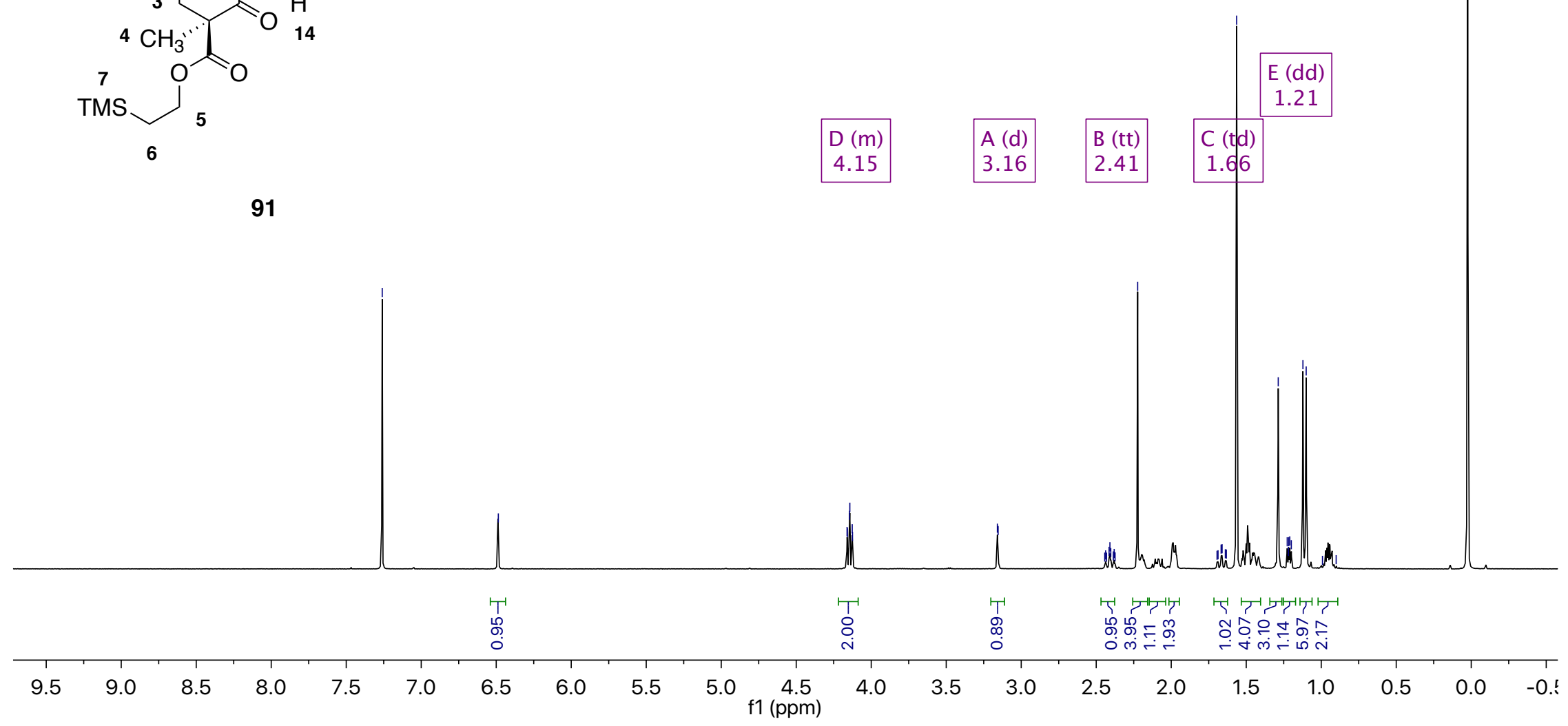
${ }^{13} \mathrm{C} \mathrm{NMR}, 125 \mathrm{MHz}, \mathrm{CDCl}_{3}$

în

\$

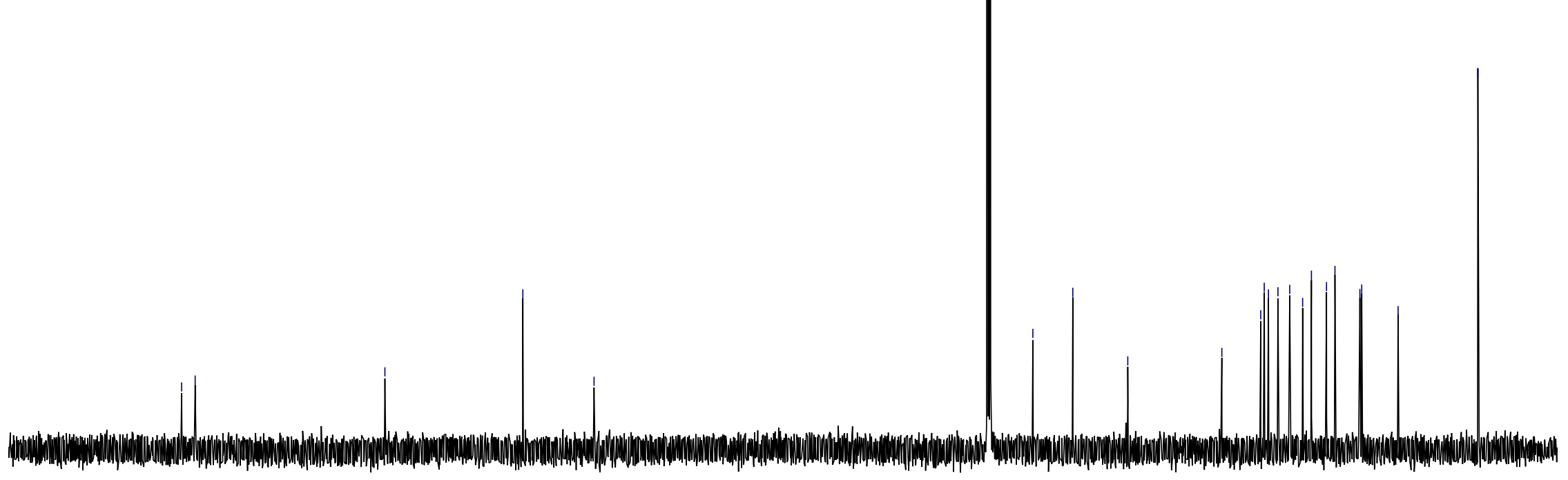

230

$\begin{array}{llll}220 & 210 \quad 200 & 190\end{array}$

180

$170 \quad 160$

150

140

130

$120 \quad 110$

90

80

60

50

$40 \quad 30$

20

10

$-10$ 


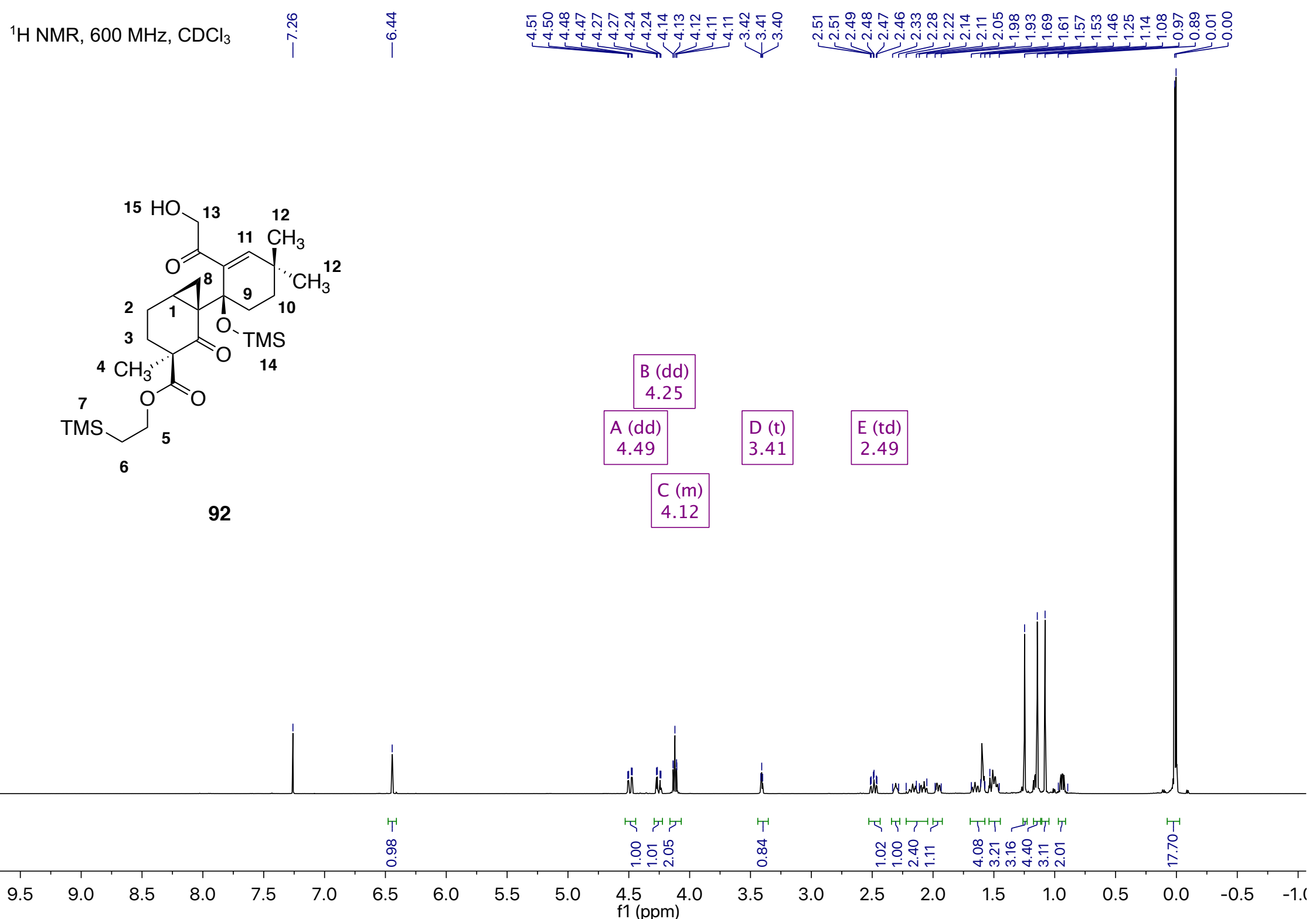


${ }^{13} \mathrm{C}$ NMR, $150 \mathrm{MHz}, \mathrm{CDCl}_{3}$

i্ণ

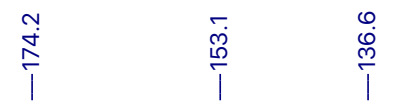

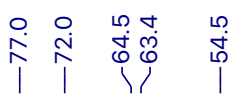

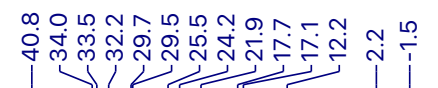

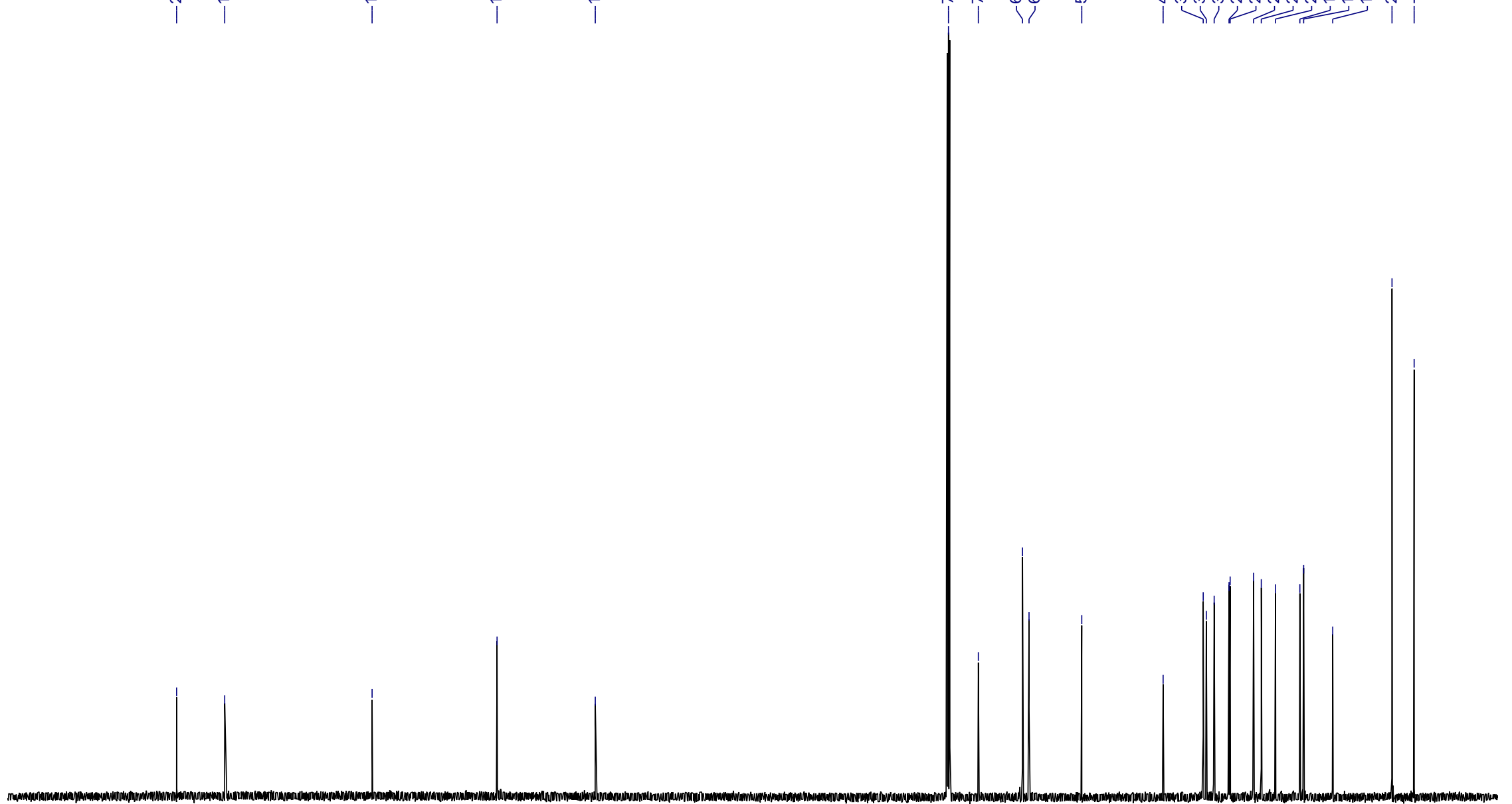

$\begin{array}{llll}230 & 220 & 210 \quad 200\end{array}$

190180

$\begin{array}{lll}170 & 160 & 150\end{array}$

140130

120

110

90

80

60

50

30

20

$10 \quad 0 \quad-10$ 
${ }^{1} \mathrm{H} \mathrm{NMR}, 500 \mathrm{MHz}, \mathrm{CDCl}_{3}$
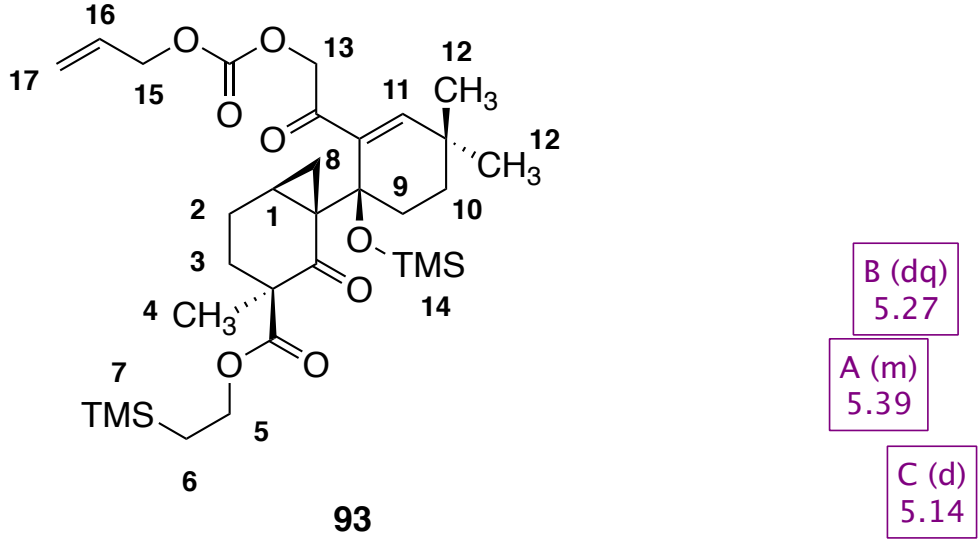

$$
\begin{array}{|c|}
\hline D(m) \\
4.12
\end{array}
$$

93

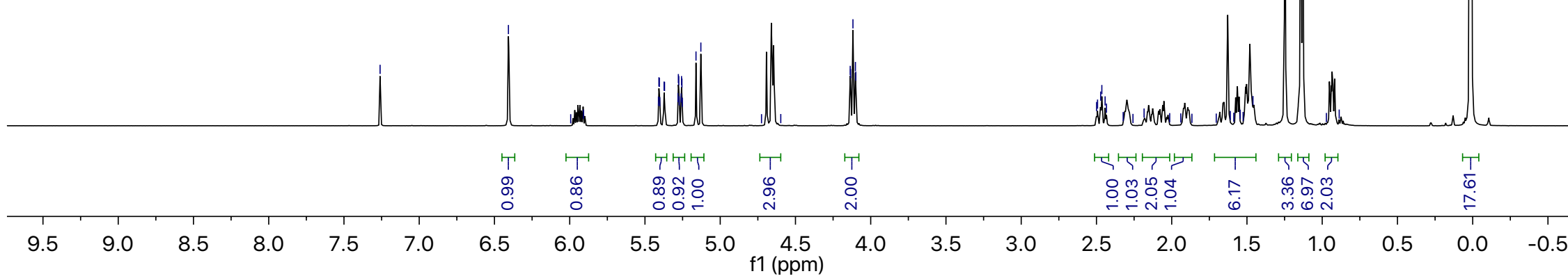


${ }^{13} \mathrm{C}$ NMR, $125 \mathrm{MHz}, \mathrm{CDCl}_{3}$

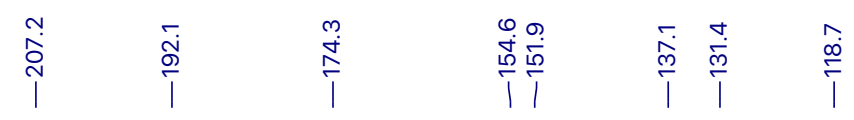

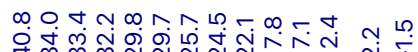

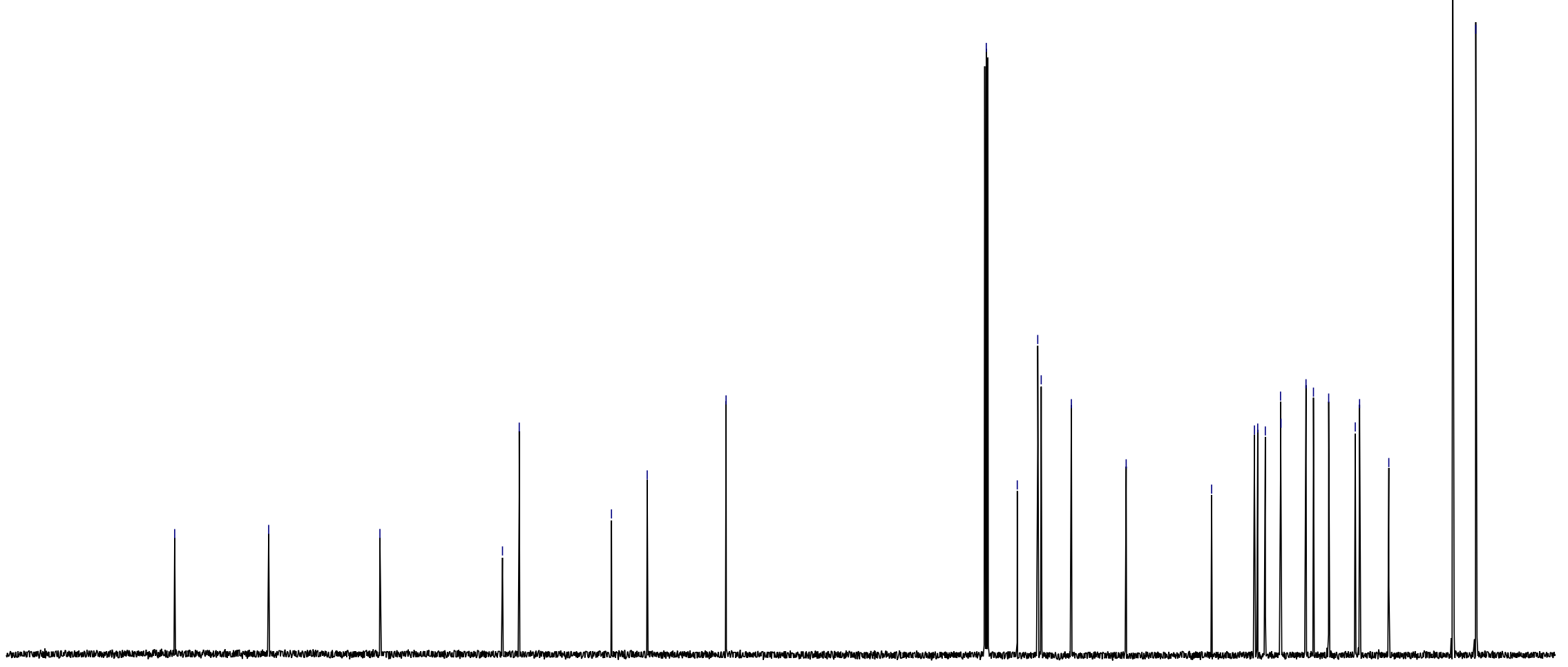


${ }^{1} \mathrm{HNMR}, 600 \mathrm{MHz}, \mathrm{CDCl}_{3} \quad \stackrel{\stackrel{0}{i}}{i} \stackrel{\infty}{i}$

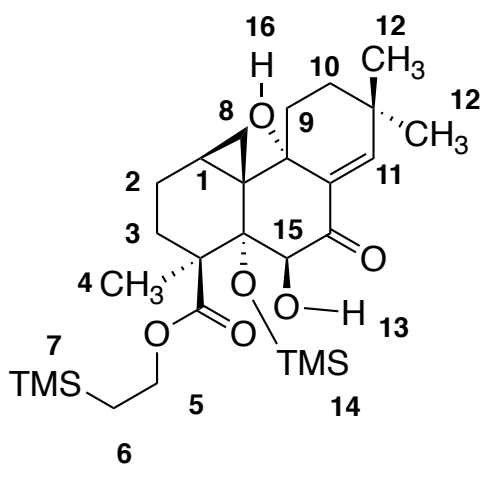

95

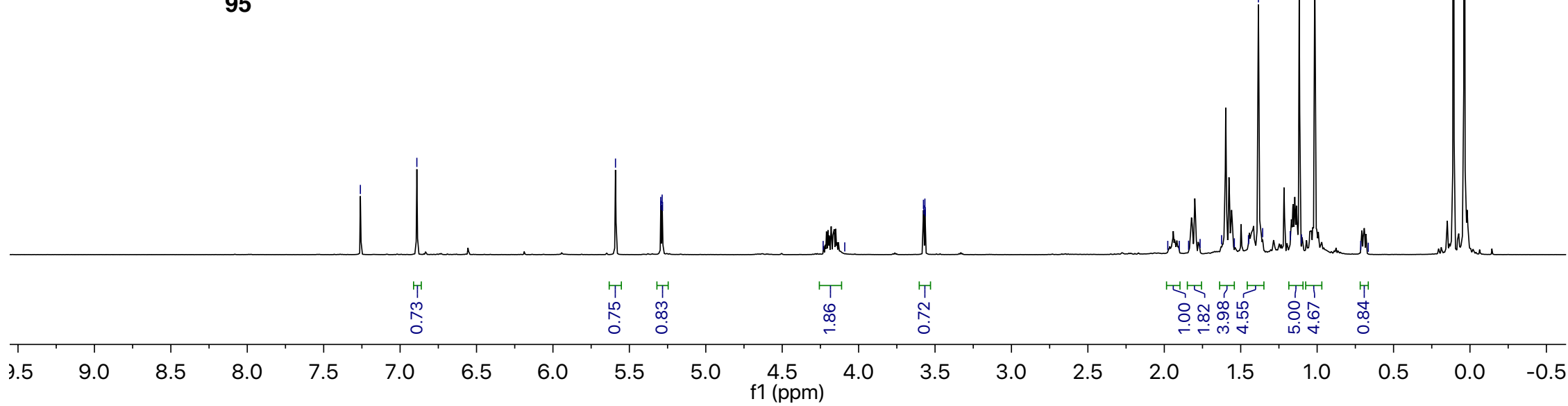


${ }^{13} \mathrm{C} \mathrm{NMR}, 150 \mathrm{MHz}, \mathrm{CDCl}_{3}$

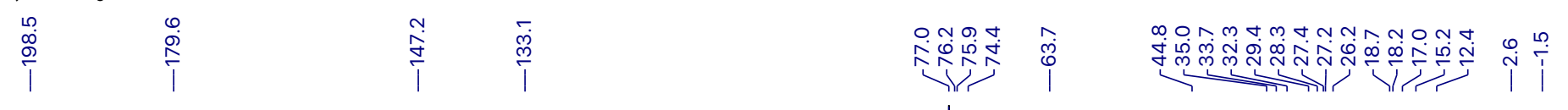

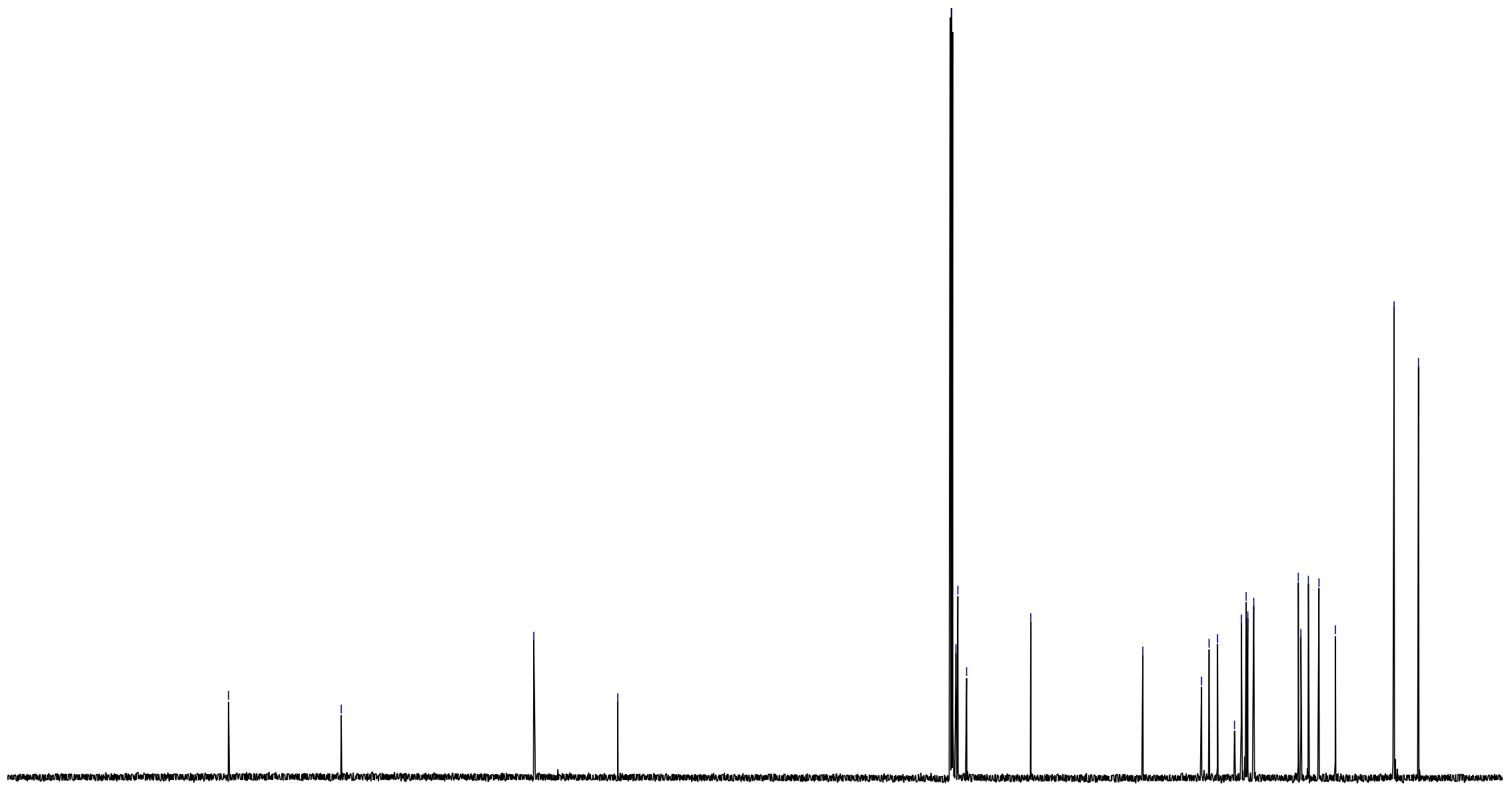

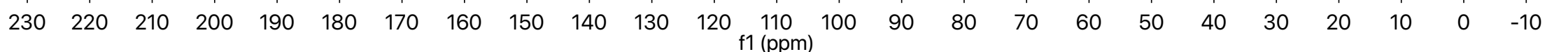




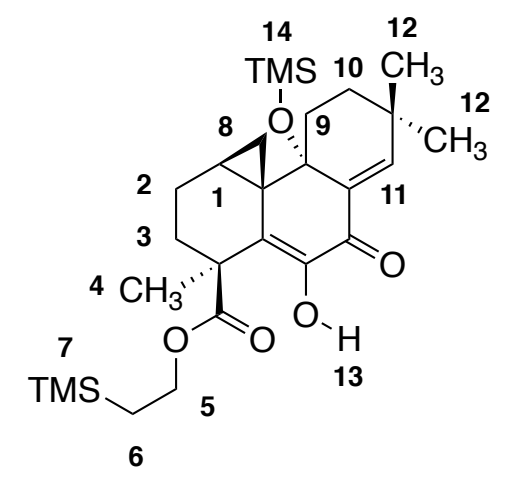

98

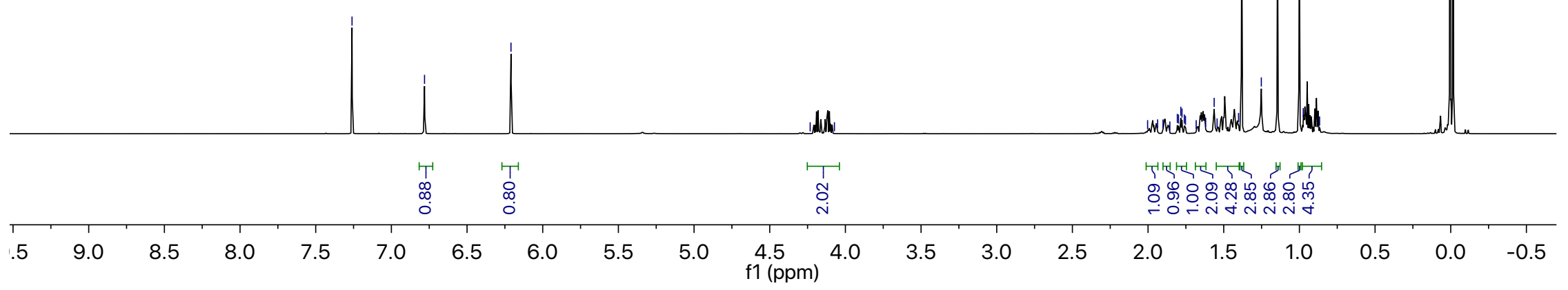




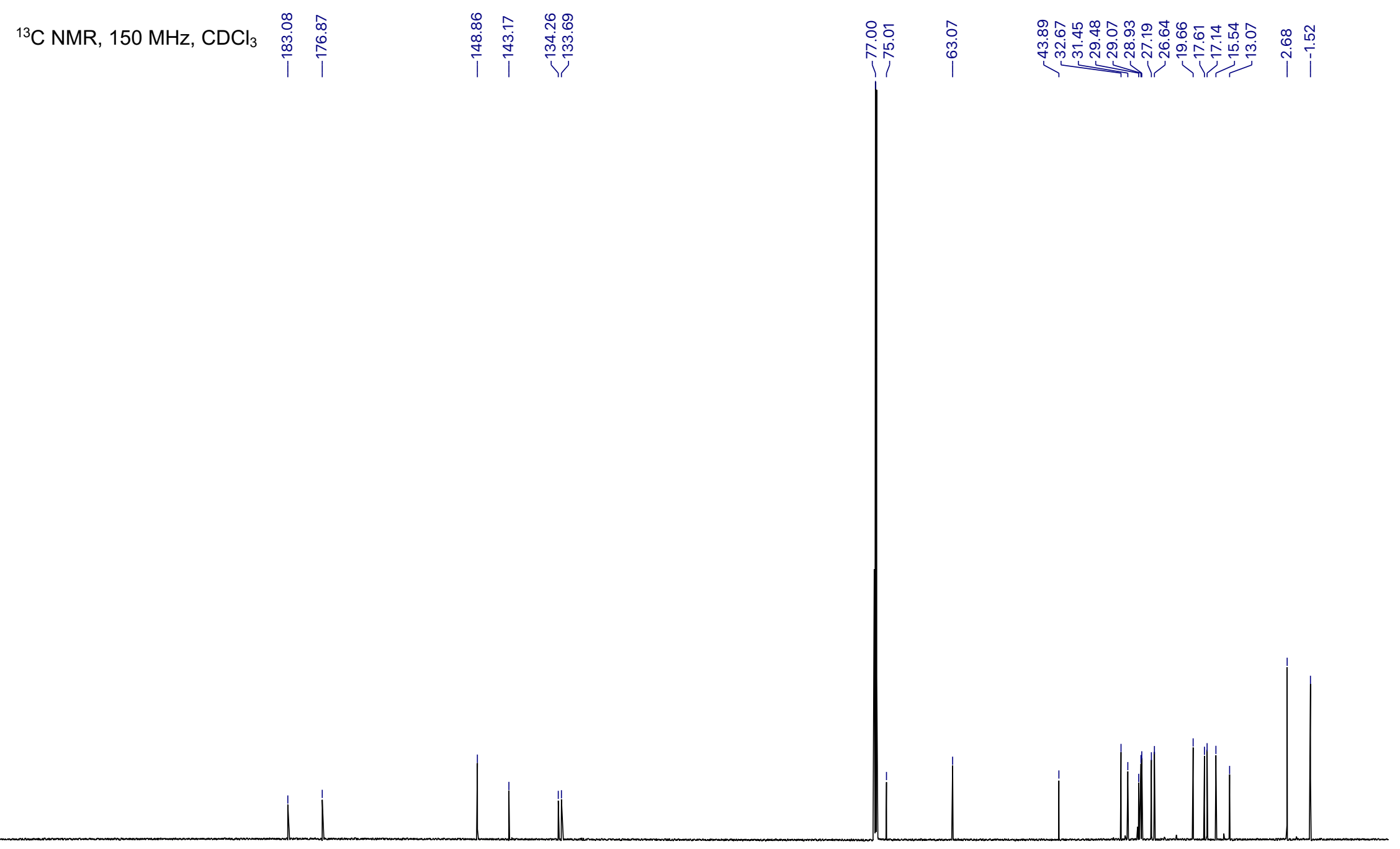

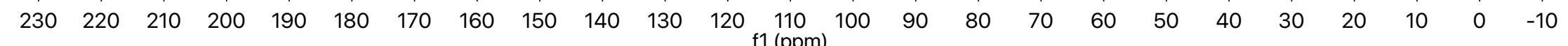




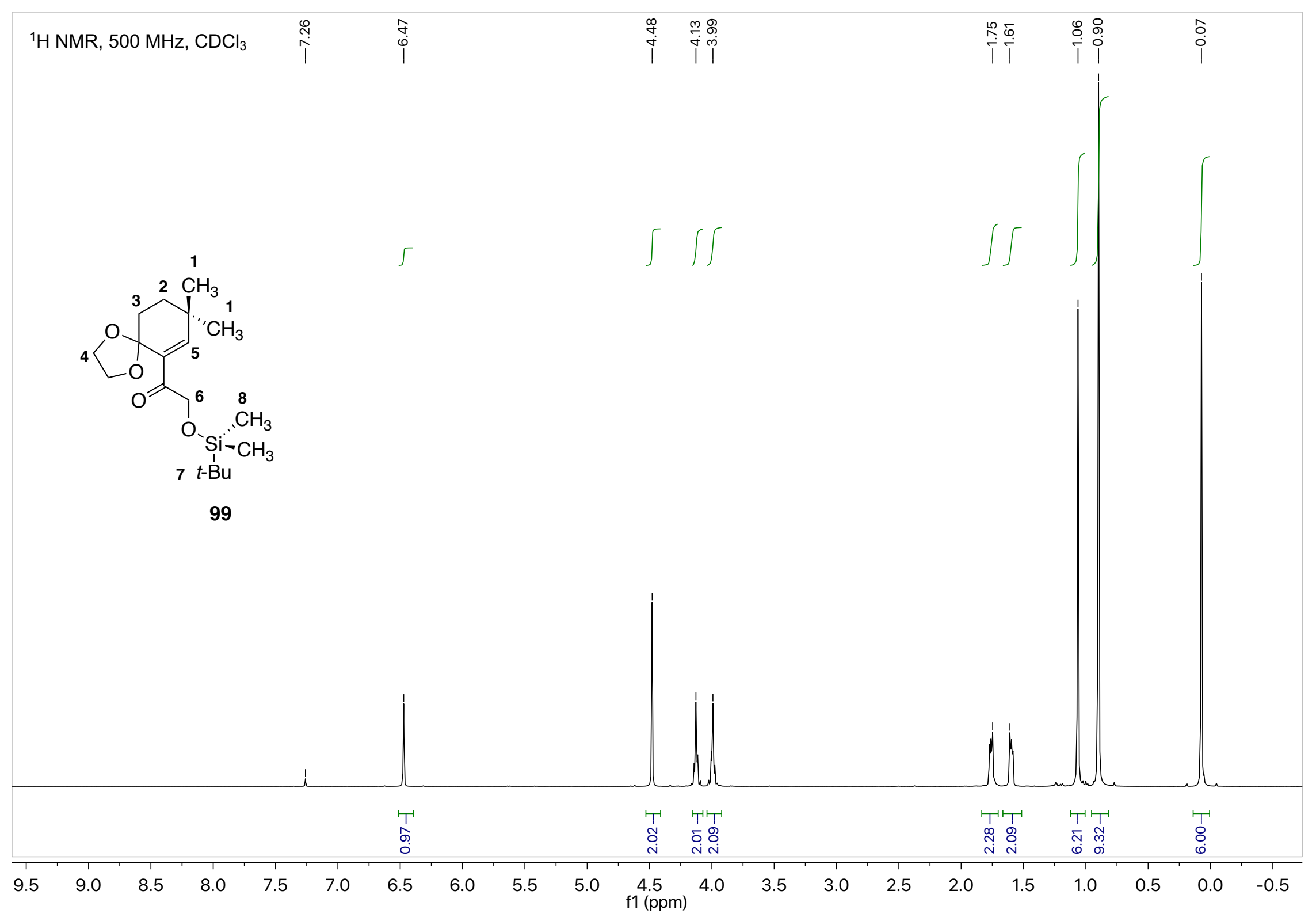


${ }^{13} \mathrm{C}$ NMR, $125 \mathrm{MHz}, \mathrm{CDCl}_{3}$

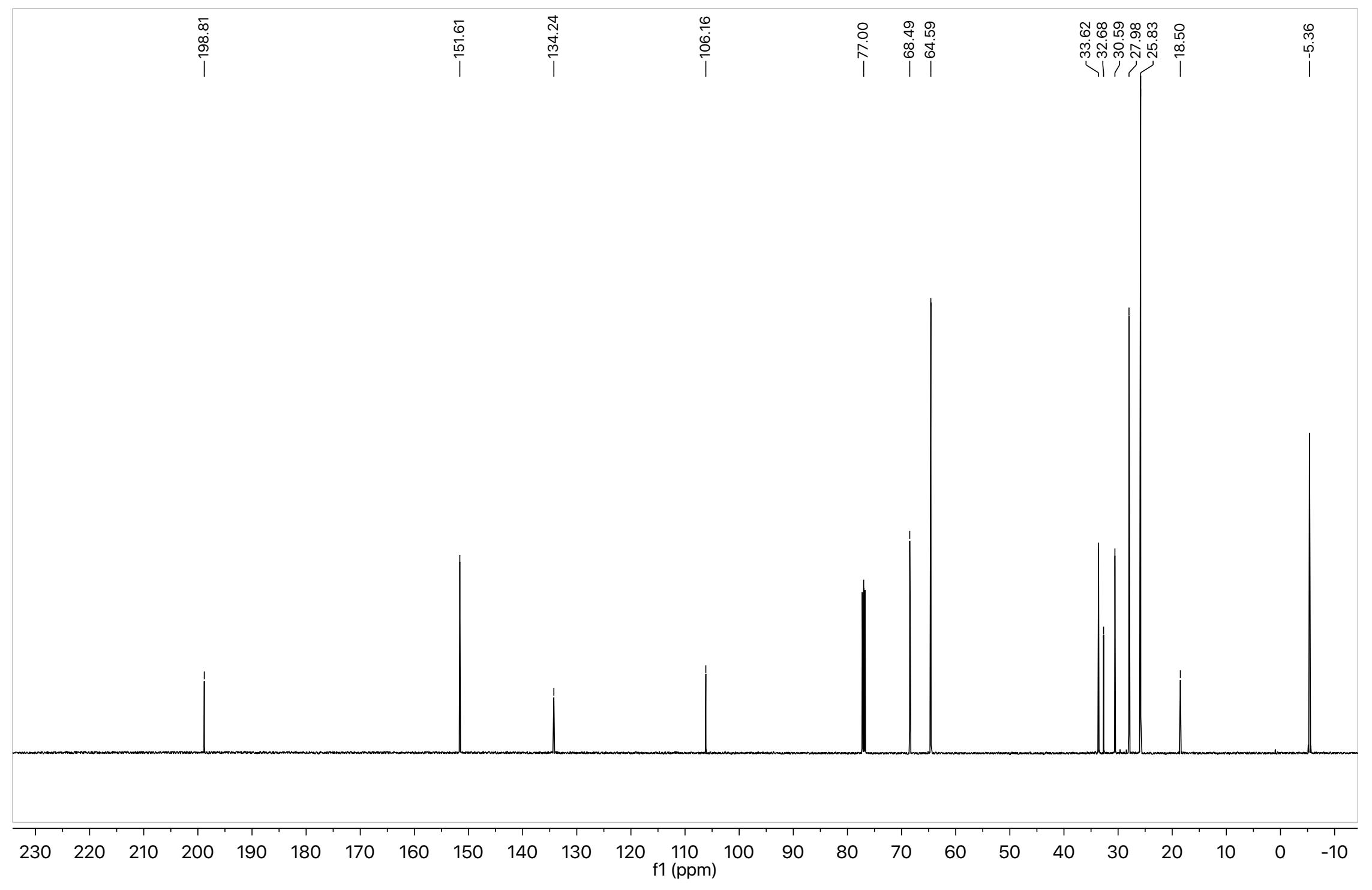




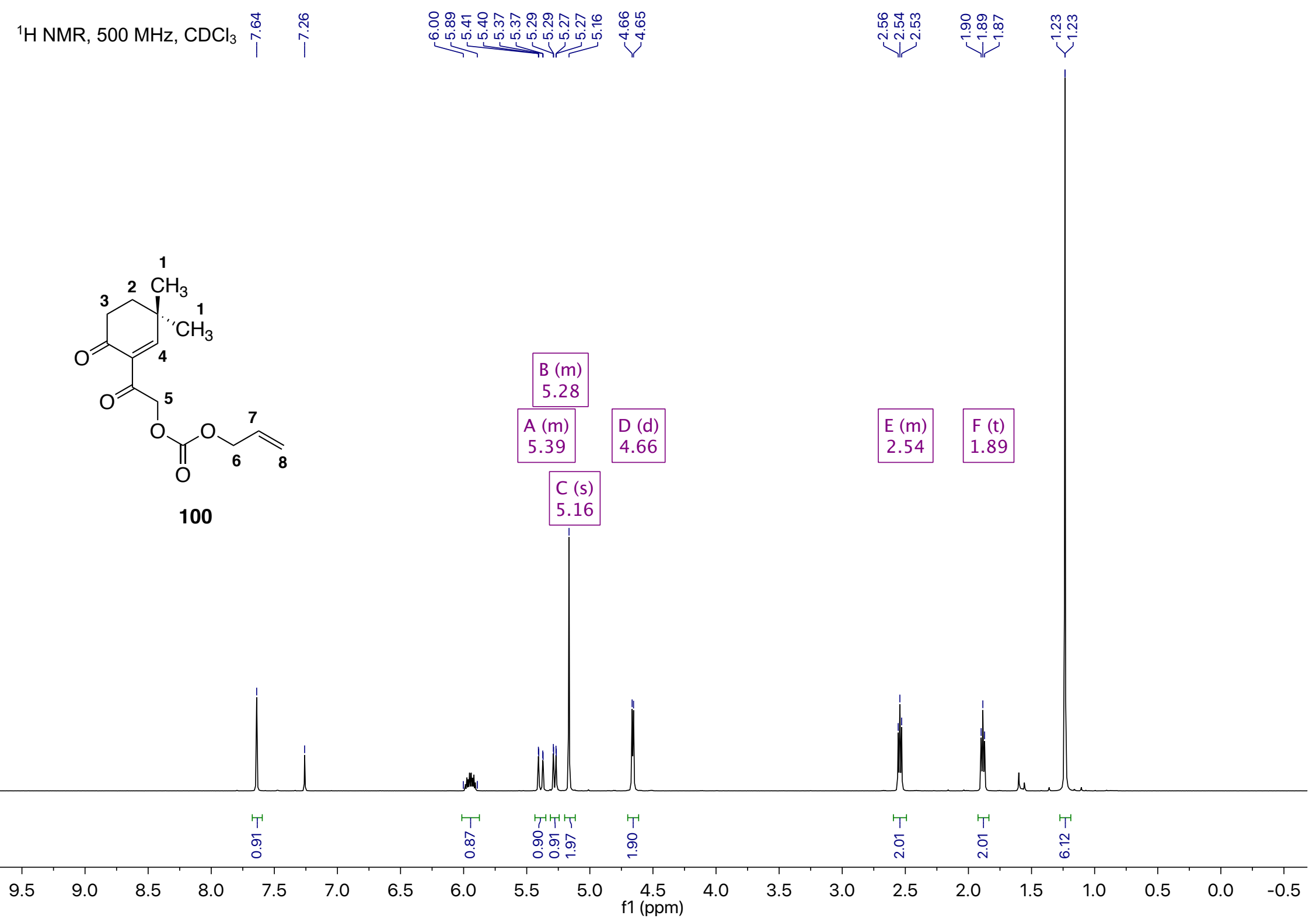


${ }^{13} \mathrm{C}$ NMR, $125 \mathrm{MHz}, \mathrm{CDCl}_{3}$

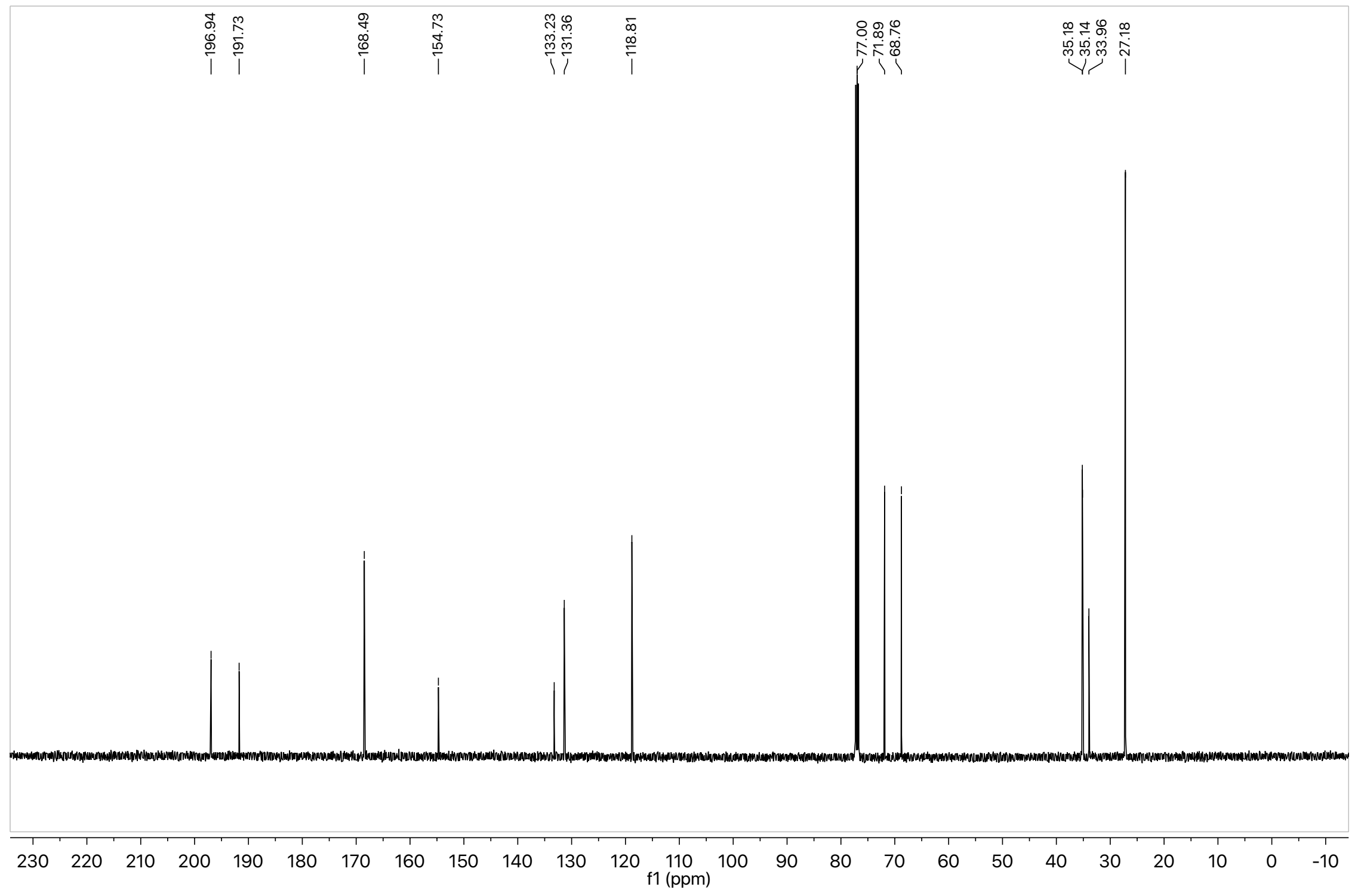


${ }^{1} \mathrm{H} N M R, 600 \mathrm{MHz}, \mathrm{C}_{6} \mathrm{D}_{6} \quad \begin{gathered}\substack{0 \\ \infty} \\ \mid\end{gathered}$

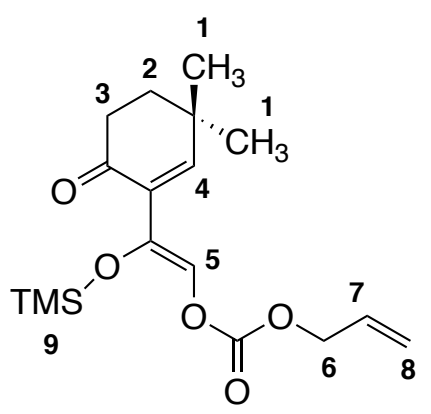

$$
\begin{array}{|l|}
\hline \text { B (d) } \\
4.89 \\
\hline A(d) \\
\hline 5.05
\end{array}
$$

101

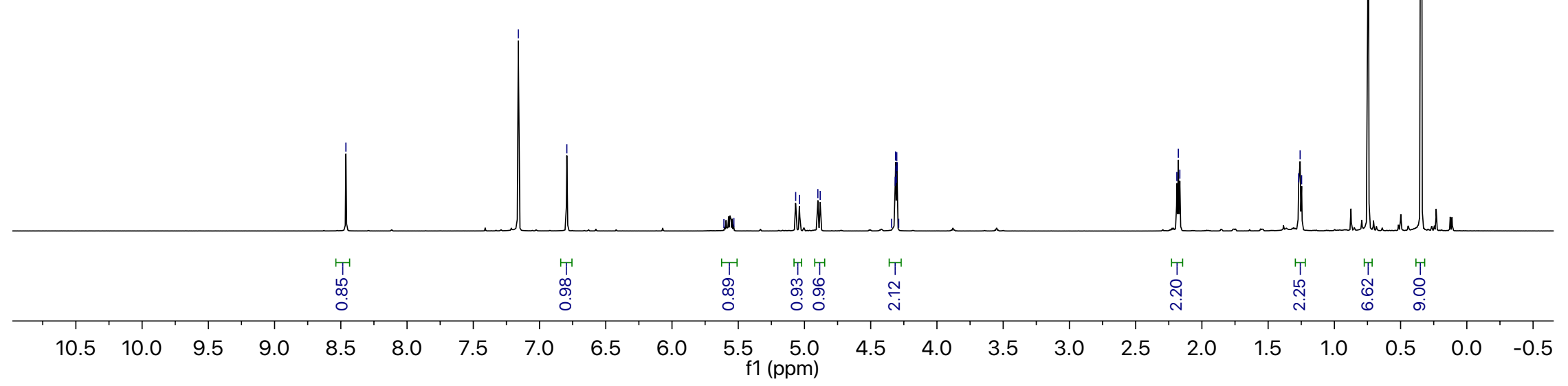


${ }^{13} \mathrm{C} N M R, 150 \mathrm{MHz}, \mathrm{C}_{6} \mathrm{D}_{6}$

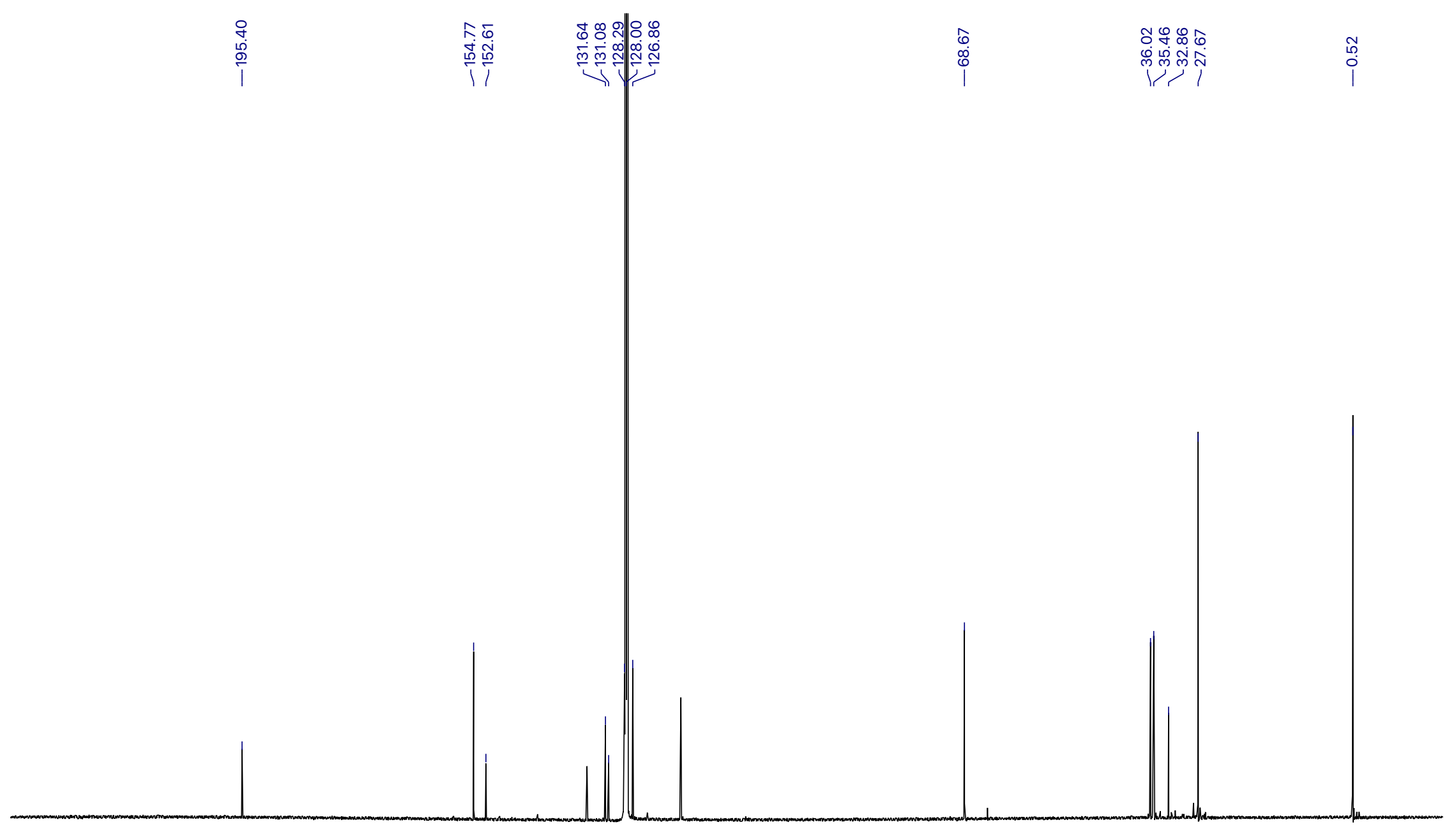

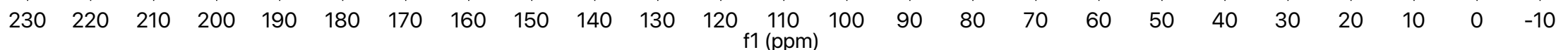


${ }^{1} \mathrm{H} \mathrm{NMR}, 600 \mathrm{MHz}, \mathrm{CDCl}_{3}$

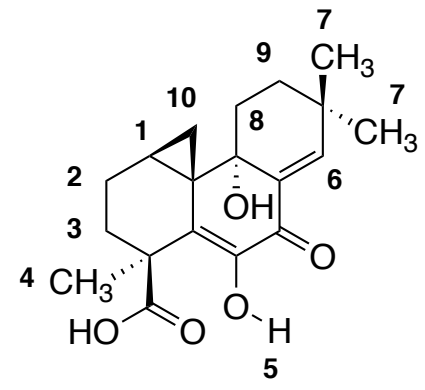

104

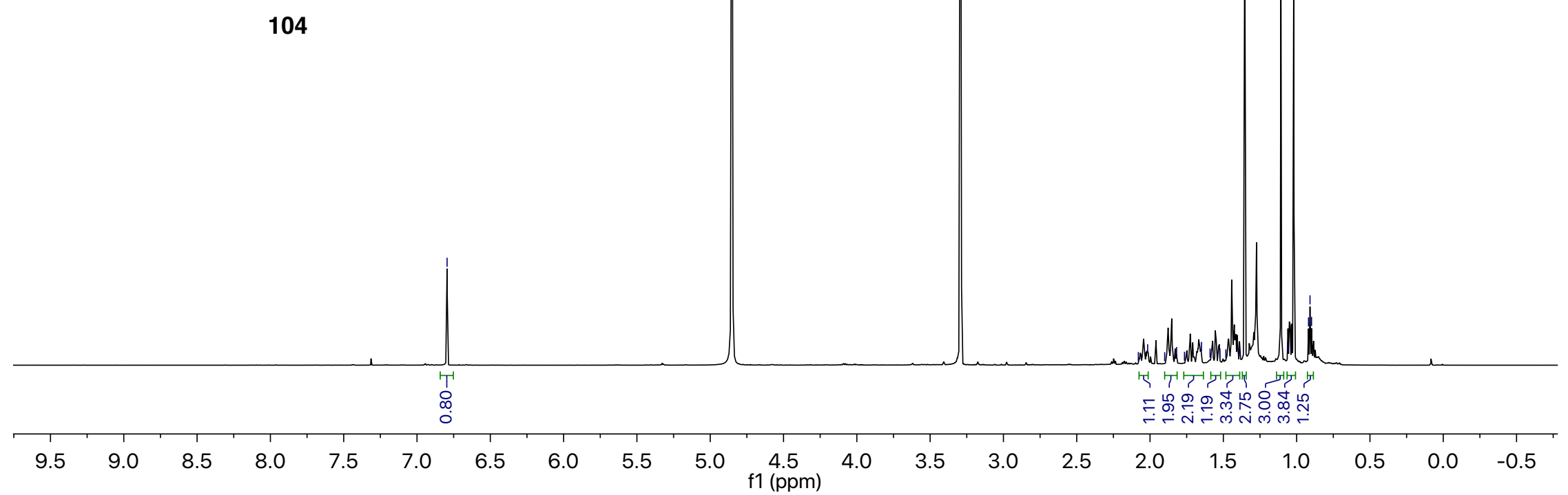




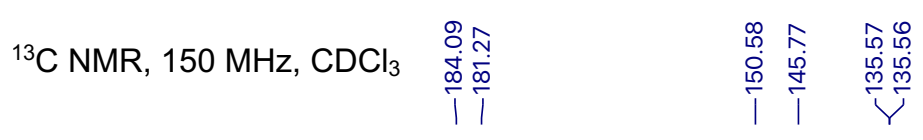

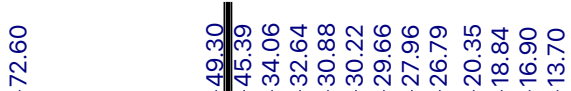

i

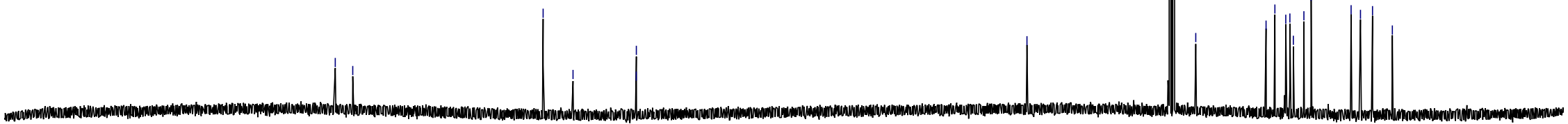

$\begin{array}{lllllllllllllllllllllllll}230 & 220 & 210 & 200 & 190 & 180 & 170 & 160 & 150 & 140 & 130 & 120 & \begin{array}{l}110 \\ \mathrm{f} 1(\mathrm{ppm})\end{array} & 100 & 90 & 80 & 70 & 60 & 50 & 40 & 30 & 20 & 10 & 0 & -10\end{array}$ 
${ }^{1} \mathrm{H} \mathrm{NMR}, 600 \mathrm{MHz}, \mathrm{CDCl}_{3}$

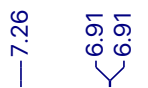

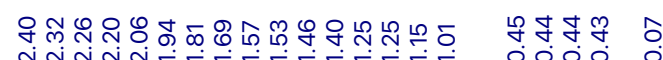

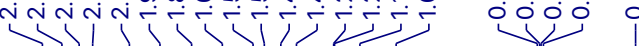

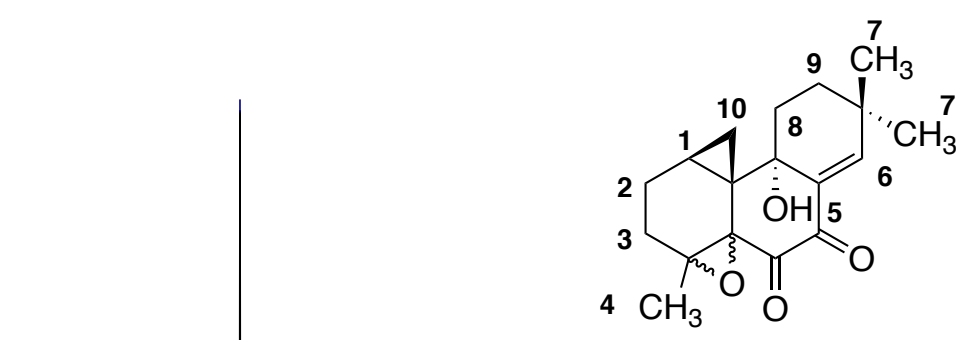

113

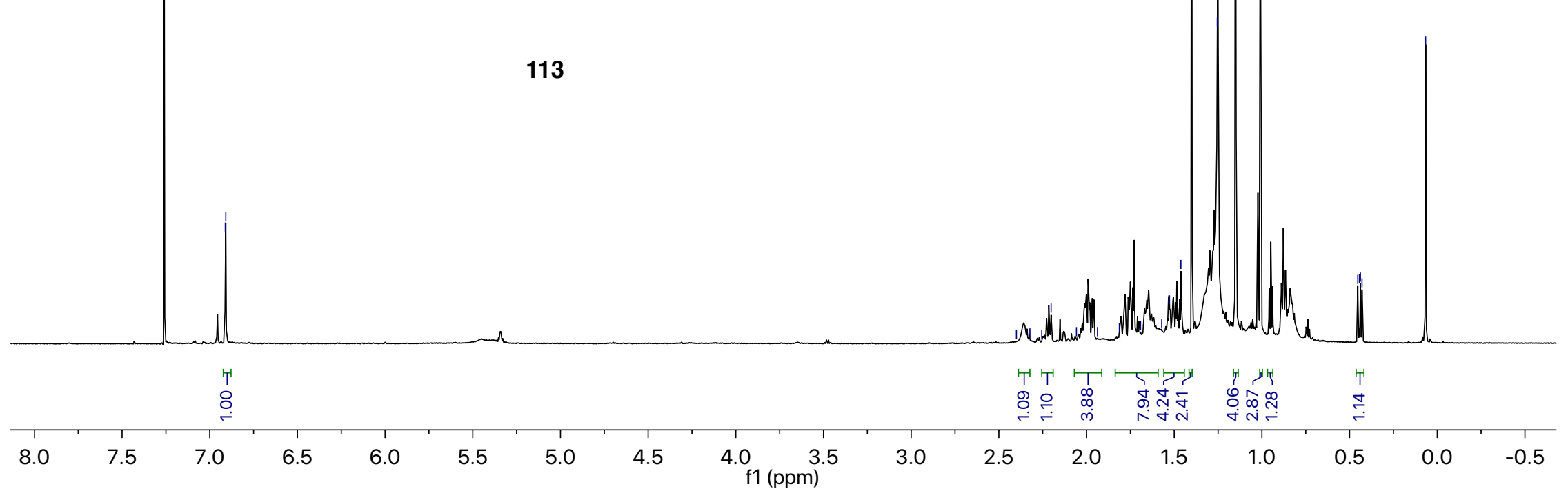


${ }^{13} \mathrm{C}$ NMR, $150 \mathrm{MHz}, \mathrm{CDCl}_{3}$

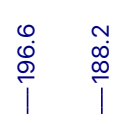

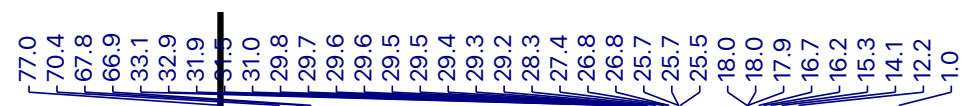

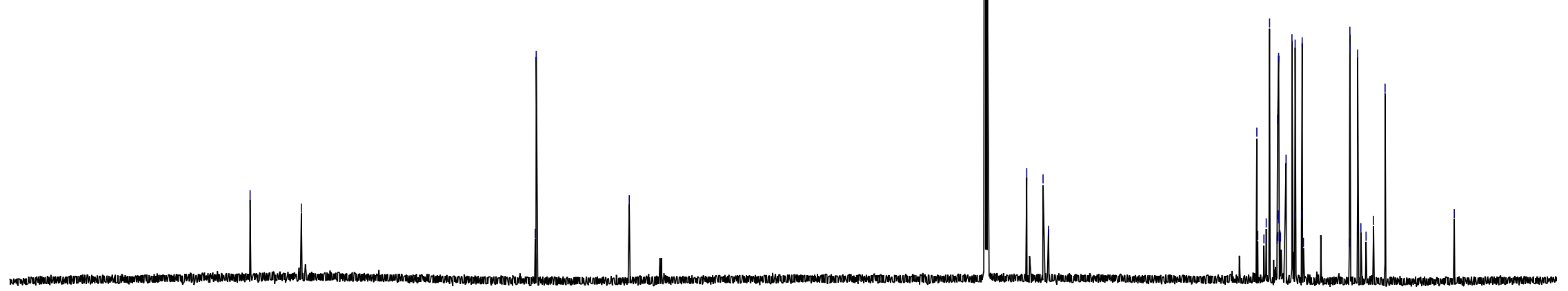

$\begin{array}{llll}230 & 220 \quad 210 \quad 200\end{array}$

$190 \quad 180$

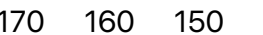

140

130

$120 \quad 110$

90

1 (ppm) 


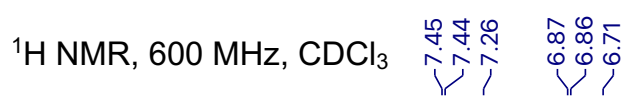

لy
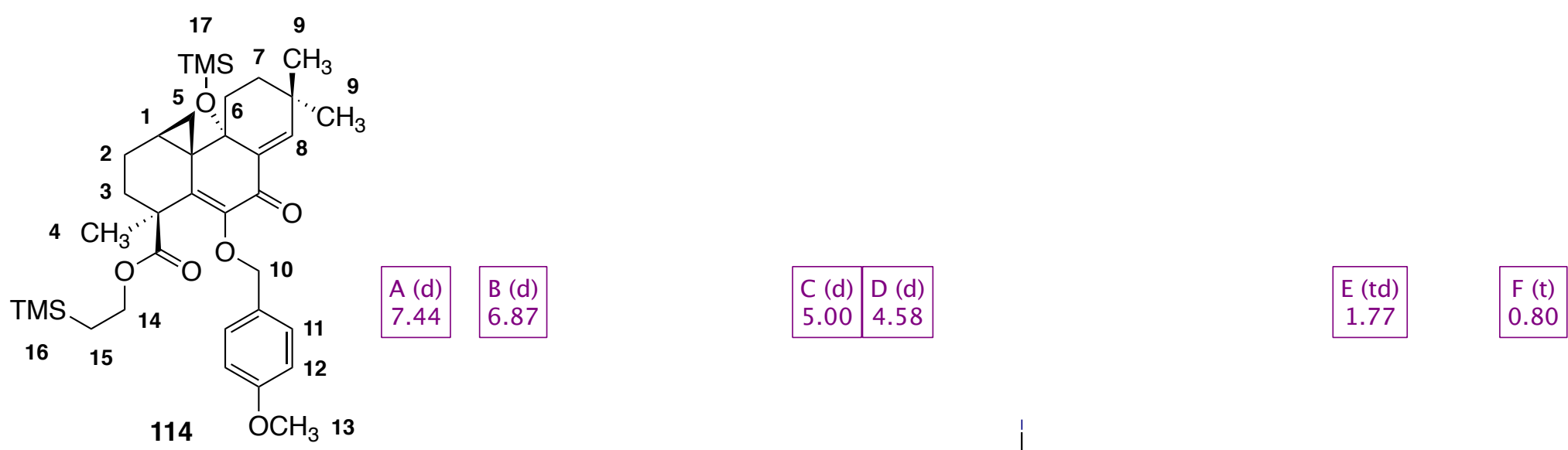

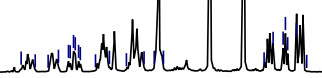

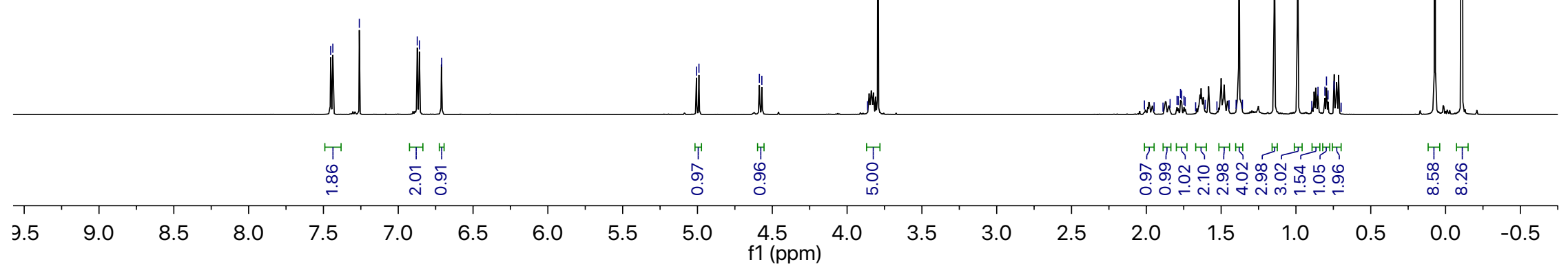




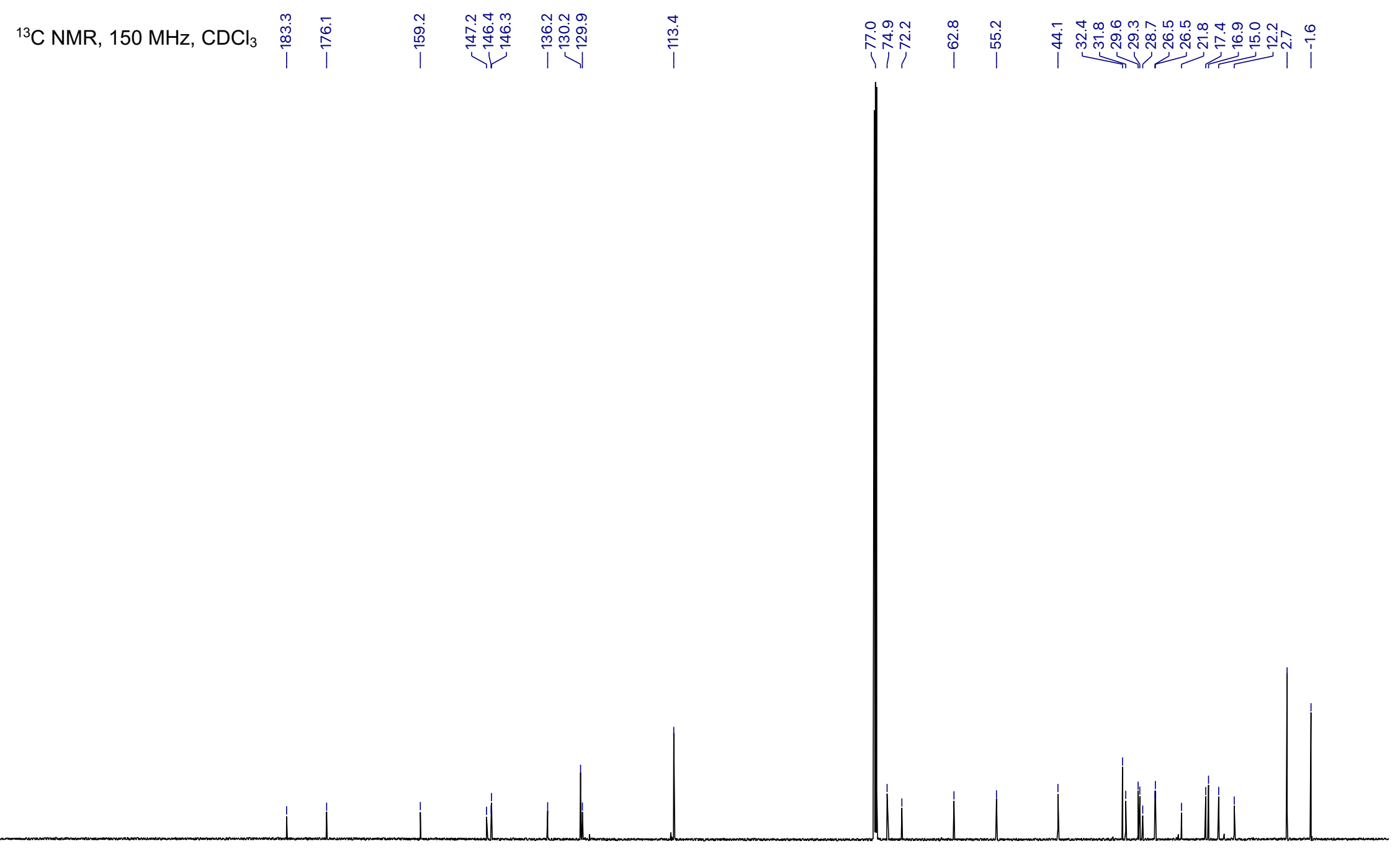

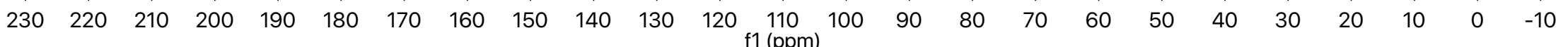




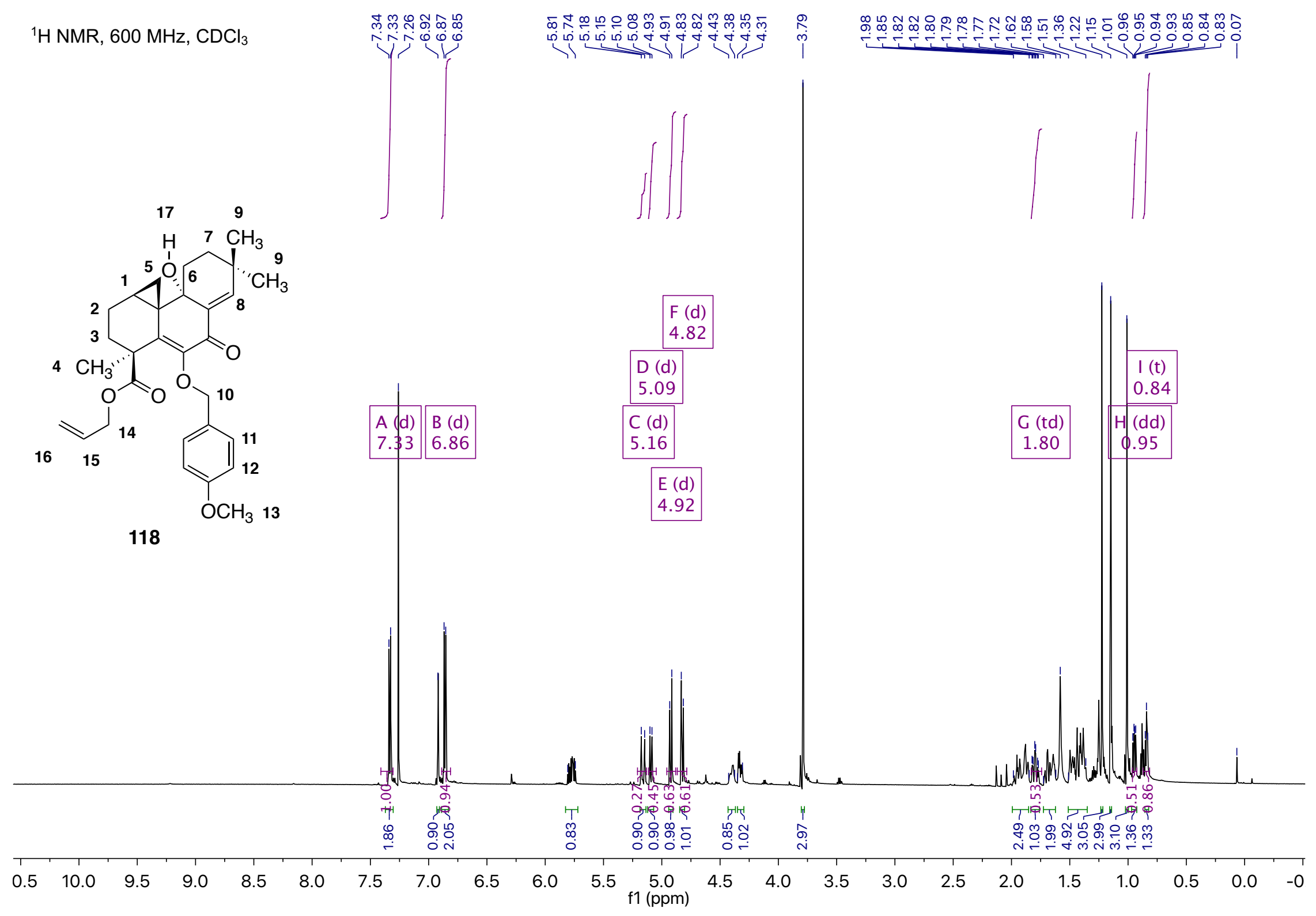




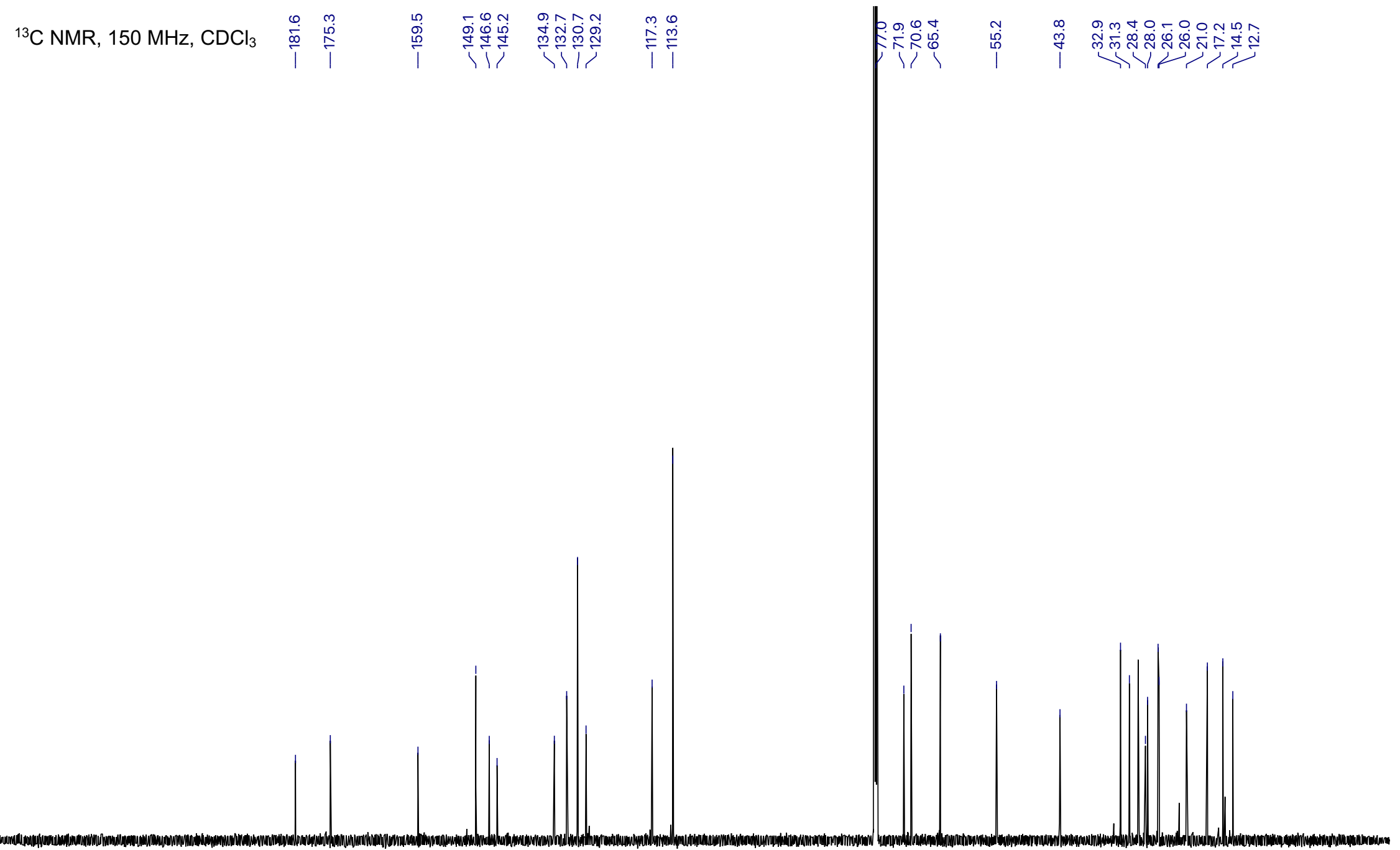

$\begin{array}{llllllllllllllllllllllllll}230 & 220 & 210 & 200 & 190 & 180 & 170 & 160 & 150 & 140 & 130 & 120 & \begin{array}{l}110 \\ \mathrm{f} 1(\mathrm{ppm})\end{array} & 100 & 90 & 80 & 70 & 60 & 50 & 40 & 30 & 20 & 10 & 0 & -10\end{array}$ 


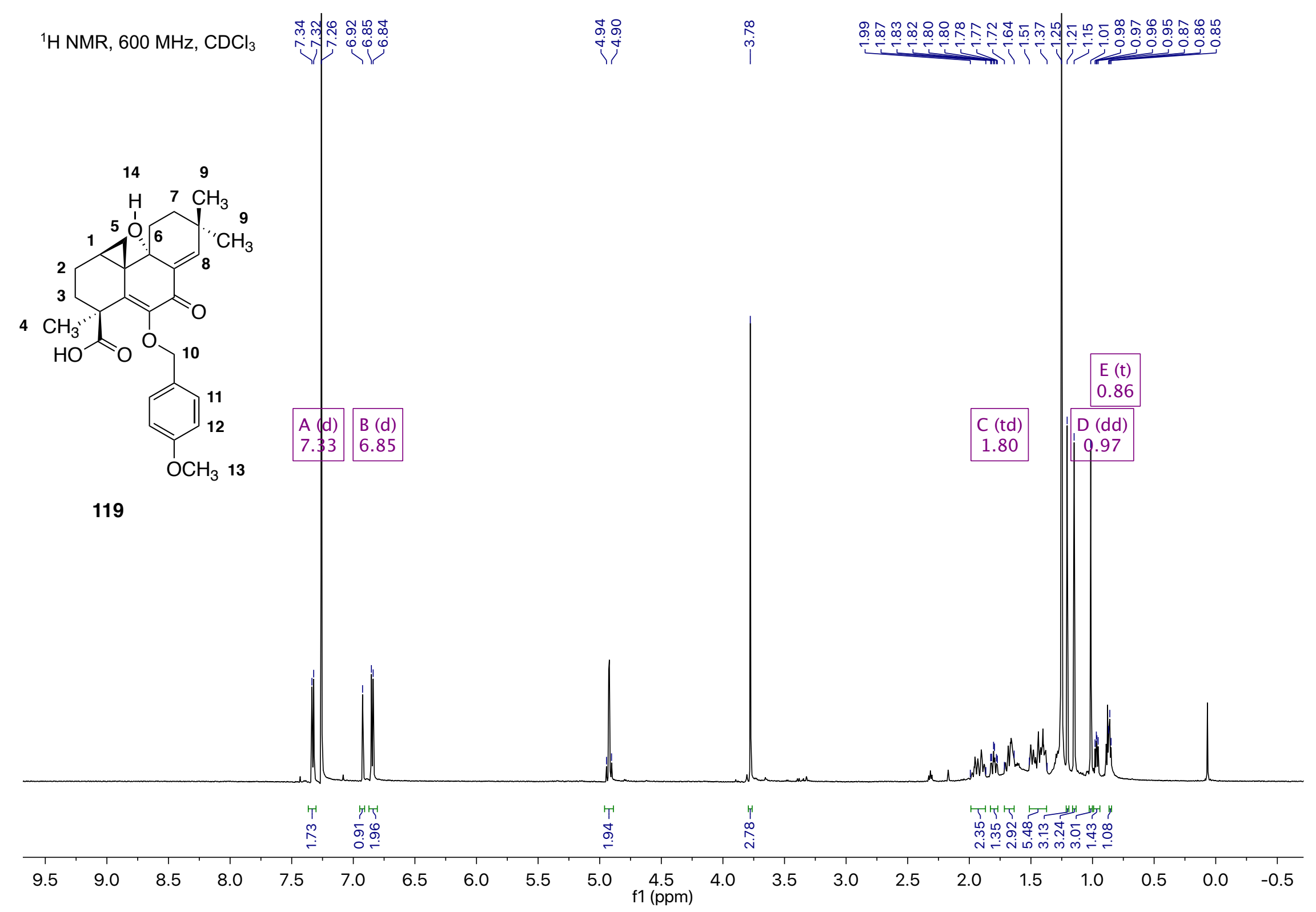




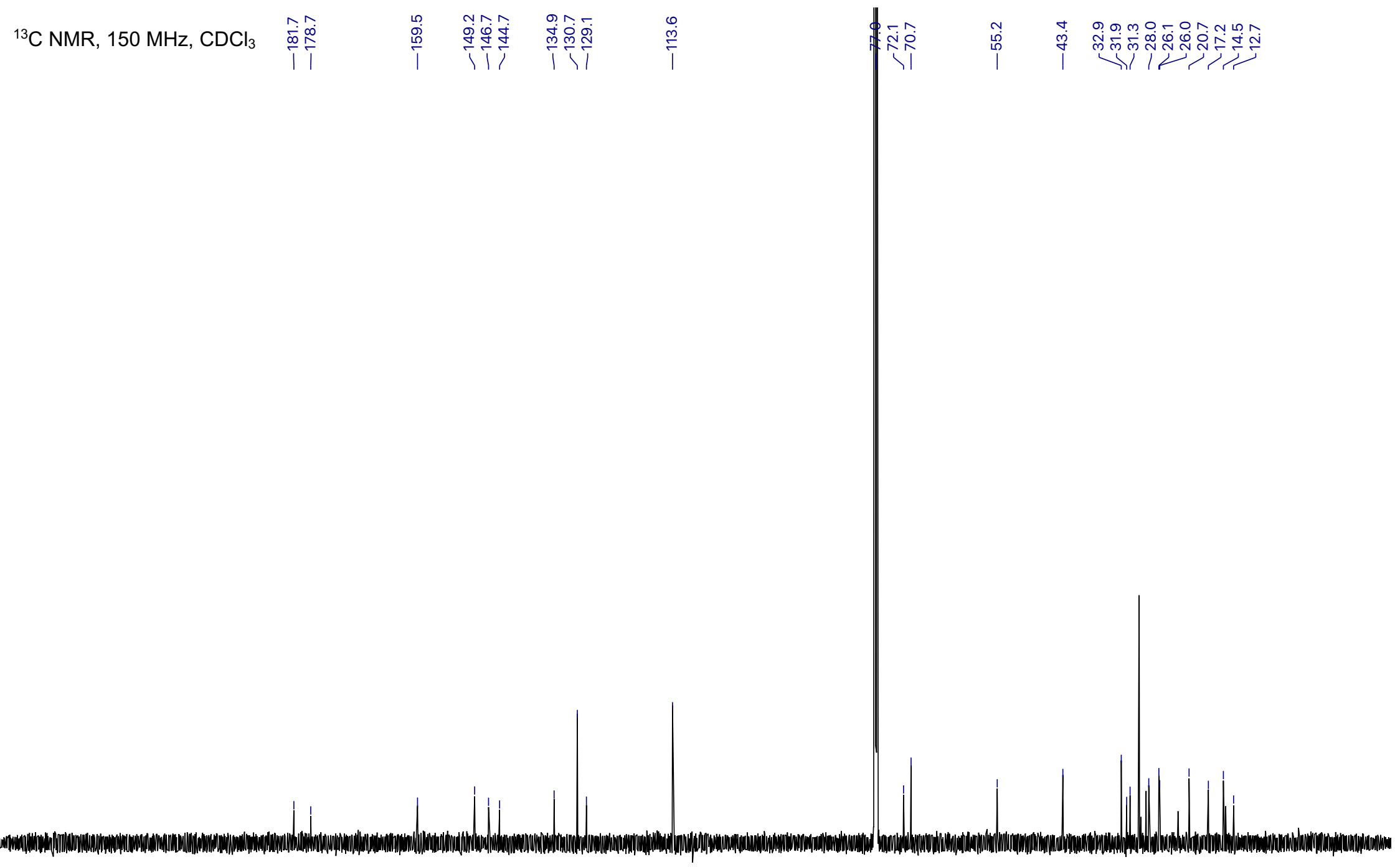

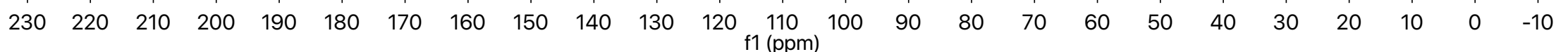


${ }^{1} \mathrm{H}$ NMR, $500 \mathrm{MHz}, \mathrm{CD}_{3} \mathrm{OD}$

$$
\stackrel{\substack{\infty \\ \text { i }}}{\infty}
$$

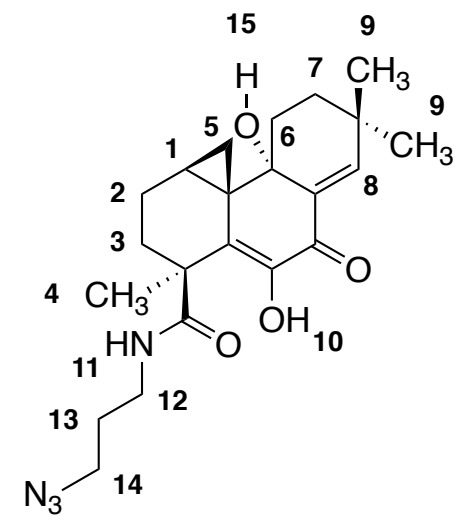

(-)-120

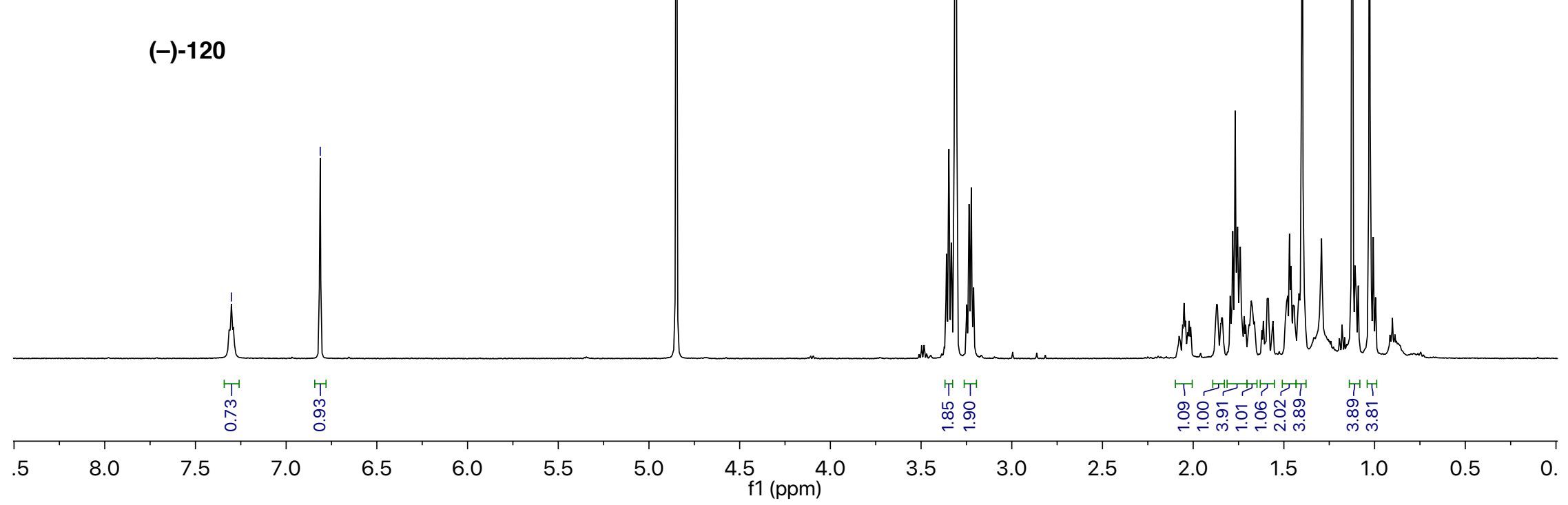


${ }^{13} \mathrm{C}$ NMR, $125 \mathrm{MHz}, \mathrm{CD}_{3} \mathrm{OD}$

广்َ

!̊ำ

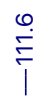

$\stackrel{\operatorname{n}}{\stackrel{i}{i}}$

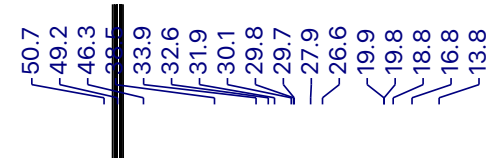

(n)

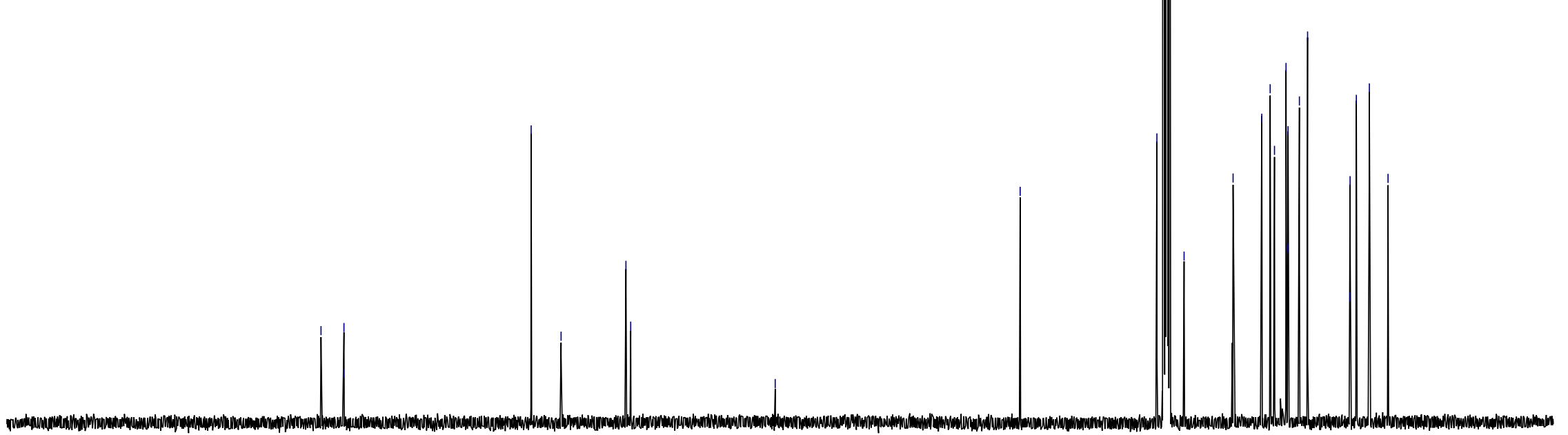

230

220210200

$190 \quad 180$

160

150

140130

$120 \quad 110$

10090

80

70

60

50

40

30

20

10

o 10

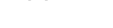


${ }^{1} \mathrm{H}$ NMR, $500 \mathrm{MHz}, \mathrm{CD}_{3} \mathrm{OD}$

$\stackrel{\substack{\infty \\ i}}{\substack{\infty \\ i}}$

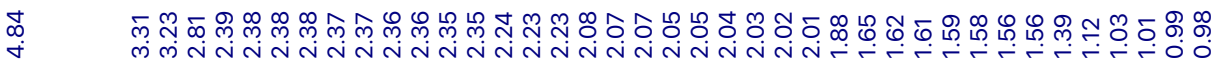

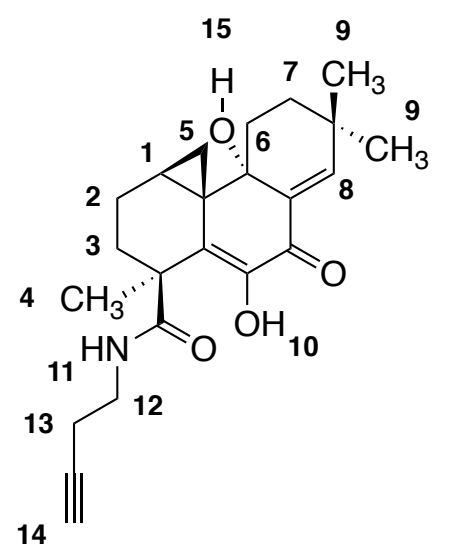

$(-)-121$

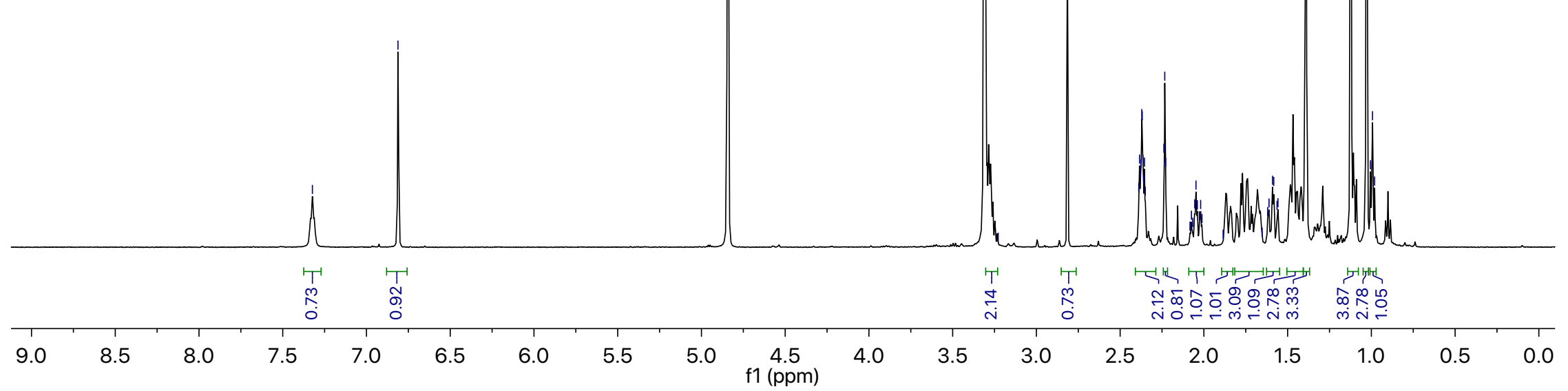


${ }^{13} \mathrm{C} N M R, 125 \mathrm{MHz}, \mathrm{CD}_{3} \mathrm{OD}$

\begin{tabular}{|c|c|}
\hline 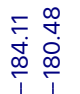 & 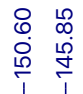 \\
\hline
\end{tabular}

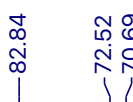

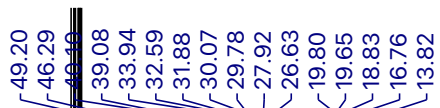

111

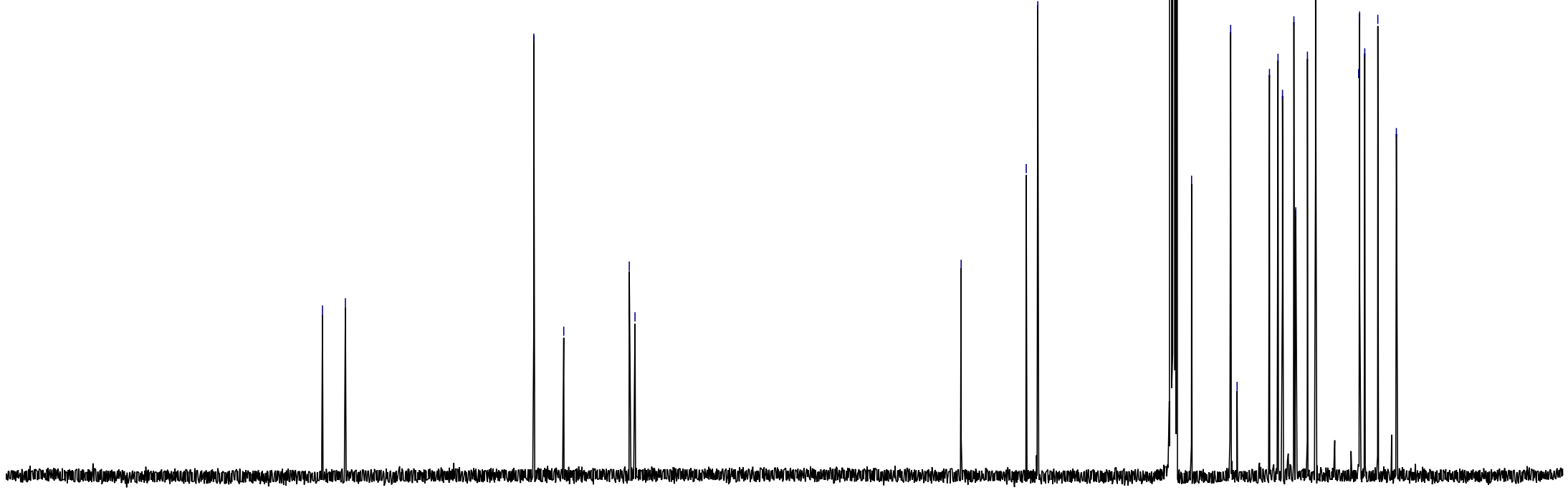

$\begin{array}{lllllllllllllllllllllllll}230 & 220 & 210 & 200 & 190 & 180 & 170 & 160 & 150 & 140 & 130 & 120 & 110 & 100 & 90 & 80 & 70 & 60 & 50 & 40 & 30 & 20 & 10 & 0 & -10\end{array}$ 


\section{Biological Studies.}

\subsection{Cell Viability Assays.}

Compounds 4, $(-)-104,(+)-104,(-)-120,(+)-120,(-)-121$, and $(+)-121$ were tested against four cell lines: HeLa, K562, LNCaP, and HCT116 for cytotoxicity. HeLa cells were obtained from Professor Craig M. Crews (Yale University). HCT-116 cells were obtained from Professor Peter M. Glazer (Yale University). K562 and LnCAP cells were obtained from Professor David Spiegel (Yale University). All cell lines were maintained in Dulbecco's Modified Eagle Medium (DMEM) and 10\% Fetal Bovine serum. Prior to an assay, frozen vials of cells were thawed and passaged one time before use. Cells $(20 \mu \mathrm{L})$ were plated into sterile black-walled, clear bottom, tissue culture treated, 384-well plates (Corning cat\#3764) using the Thermo CombiiDrop. All cell lines were plated at 400 cells/well. Plates were then centrifuged at $46 \mathrm{~g}$ for $10 \mathrm{sec}$ and incubated overnight at $37^{\circ} \mathrm{C}$ in a humidified $5 \% \mathrm{CO}_{2}$ incubator. Compound stock solutions of 50 or $25 \mathrm{mM}$ were diluted to intermediate dilutions in DMSO. Compounds $(40 \mathrm{~nL})$ was transferred from the compound source plate to the cell assay plates using a Labcyte Echo acoustic dispenser as a 14point dilution curve in duplicate. The same volume of DMSO was added to the negative control wells. The final concentration of compound was $100-0.012 \mu \mathrm{M}$ for compounds with stock concentrations of $50 \mathrm{mM}$ or $50-0.06 \mu \mathrm{M}$ for compounds with stock concentrations of $25 \mathrm{mM}$. The final DMSO concentration was $0.02 \%$. Tamoxifen $(60 \mu \mathrm{M}$ final added in $120 \mathrm{~nL}$ from a 10 $\mathrm{mM}$ stock) was added as a positive control. Assay plates were centrifuged at $46 \mathrm{~g}$ for $10 \mathrm{sec}$ and incubated for $72 \mathrm{~h}$ at $37^{\circ} \mathrm{C}$ in a humidified $5 \% \mathrm{CO}_{2}$ incubator. CellTiter-Glo (Promega) was used to measure cell viability in the assay wells according to the manufacturer's instructions. After addition of the CellTiter-Glo reagent to the assay plates using the MultiDrop dispenser, the plates were shaken on a Thermomixer $\mathrm{R}$ (Eppendorf) at 1,100 rpm for $1 \mathrm{~min}$ and incubated in the dark for $30 \mathrm{~min}$ at room temperature. Luminescence was measured in the PheraStar plate reader with 0.3 second sampling time per well. Wells displaying cytotoxicity have lower luminescence signals relative to the vehicle control wells. Raw data (luminescence counts per second) were normalized to Percent Effect by the formula 100 - (((sample - positive control mean) / (negative control mean - positive control mean))* 100). 


\subsection{DNA cleavage assay of 4 .}

The 4361 bp pBR322 vector (circular) was purchased from New England Biolabs ${ }^{\circledR}$ (Ipswich, MA). To set up every reaction, $200 \mathrm{ng}$ of circular pBR322 DNA (15.2 $\mu \mathrm{M}$ base pairs) was incubated with 4 in concentration from $500 \mu \mathrm{M}$ to $10 \mathrm{nM}$ in a final concentration of $20 \mu \mathrm{L}$. 4 was diluted in DMSO such that each reaction contained 5\% DMSO concentration. Reactions were conducted in either TE buffer (10 mM Tris, $1 \mathrm{mM}$ EDTA, pH 8.0) or phosphate buffer (10 mM sodium phosphate, $\mathrm{pH}$ 7.0). Pure DMSO vehicle was used as negative control prepared simultaneously. The negative control employs $200 \mathrm{ng}$ of circular pBR322 DNA (15.2 $\mu \mathrm{M}$ base pairs) treated with pure DMSO vehicle in either TE buffer (10 mM Tris, 1 mM EDTA, pH 8.0) or phosphate buffer (10 mM sodium phosphate, $\mathrm{pH} 7.0)$ with a final DMSO concentration of $5 \%$. Reactions proceeded for $16 \mathrm{~h}$ at $37^{\circ} \mathrm{C}$, and the reacted DNA was subjected to electrophoretic analysis.

During gel electrophoresis, linearized pBR322 DNA was used as positive control. To prepare the linearized DNA, the 4361 bp pBR322 vector (New England Biolabs ${ }^{\circledR}$ ) was linearized with 20 units $/ \mu \mathrm{g}$ EcoRI-HF® (New England Biolabs ${ }^{\circledR}$ ) and the linearized DNA was purified using a PCR clean-up kit (New England Biolabs $\left.{ }^{\circledR}\right)$, eluted into $10 \mathrm{mM}$ Tris (pH 8.0), and quantified using a nanodrop. For each DNA sample (except the linearized pBR322 positive control), 10 microliters (100 ng) of DNA was removed and mixed with $2 \mu \mathrm{L}$ of $6 \times$ purple gel loading dye; no SDS (New England Biolabs ${ }^{\circledR}$ ), and loaded onto $1 \%$ agarose Tris Borate EDTA (TBE) gels. The gel electrophoresis was conducted at $90 \mathrm{~V}$ for $1.5 \mathrm{~h}$. The gel was stained with SybrSafe DNA gel stain (Thermo Fisher) for $2 \mathrm{~h}$ before imaging.

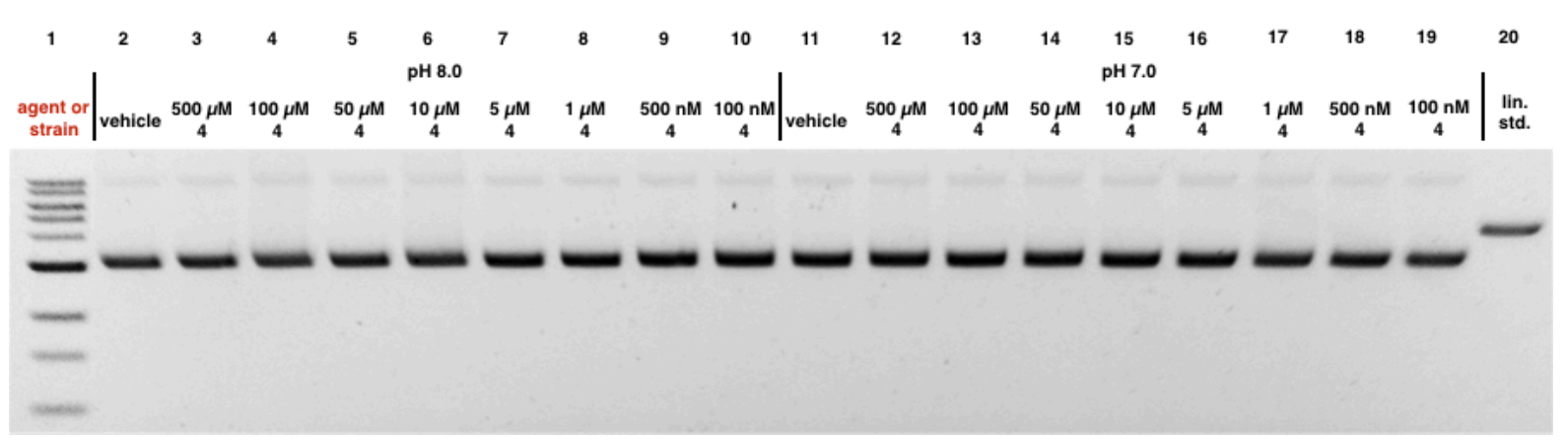

Figure S1: DNA plasmid cleavage assay employing circular pBR322 plasmid DNA and 4. 5\% DMSO was used as vehicle (negative control), and linearized pBR322 DNA was used as positive control. DNA ladder (Lane \#1); 5\% DMSO, pH 8.0 (Lane \#2); $500 \mu \mathrm{M} \mathrm{4,} \mathrm{pH} 8.0$ (Lane \#3); 100 $\mu \mathrm{M} 4$, pH 8.0 (Lane \#4); $50 \mu \mathrm{M}$ 4, pH 8.0 (Lane \#5); $10 \mu \mathrm{M} \mathrm{4,} \mathrm{pH} \mathrm{8.0} \mathrm{(Lane} \mathrm{\# 6);} 5 \mu \mathrm{M} \mathrm{4,} \mathrm{pH} \mathrm{8.0}$ (Lane \#7); $1 \mu \mathrm{M} \mathrm{4,pH} 8.0$ (Lane \#8); $500 \mathrm{nM} \mathrm{4,} \mathrm{pH} 8.0$ (Lane \# 9); $100 \mathrm{nM} \mathrm{4,} \mathrm{pH} 8.0$ (Lane \#10); 5\% DMSO, pH 7.0 (Lane \#11); $500 \mu \mathrm{M}$ 4, pH 7.0 (Lane \#12); $100 \mu \mathrm{M}$ 4, pH 7.0 (Lane \#13); 50 $\mu \mathrm{M} 4$, pH 7.0 (Lane \#14); $10 \mu \mathrm{M} 4$, pH 7.0 (Lane \#15); $5 \mu \mathrm{M} 4$, pH 7.0 (Lane \#16); $1 \mu \mathrm{M} \mathrm{4,} \mathrm{pH}$ 7.0 (Lane \#17); $500 \mathrm{nM} \mathrm{4,} \mathrm{pH} \mathrm{7.0} \mathrm{(Lane} \mathrm{\# 18);} 100 \mathrm{nM} \mathrm{4,} \mathrm{pH} \mathrm{7.0} \mathrm{(Lane} \mathrm{\# 19),} \mathrm{linearized} \mathrm{pBR322}$ DNA (Lane \#20). Conditions (Lane \#2): circular pBR322 DNA (15.2 $\mu \mathrm{M}$ in base pairs), $5 \%$ DMSO (vehicle), TE buffer (10 mM Tris, $1 \mathrm{mM}$ EDTA, pH 8.0), $16 \mathrm{~h}, 37^{\circ} \mathrm{C}$. Conditions (Lanes \#3-\#10): circular pBR322 DNA (15.2 $\mu \mathrm{M}$ in base pairs), $4(500 \mu \mathrm{M}-100 \mathrm{nM}), 5 \% \mathrm{DMSO}$, TE buffer (10 mM Tris, $1 \mathrm{mM}$ EDTA, pH 8.0), $16 \mathrm{~h}, 37^{\circ} \mathrm{C}$. Conditions (Lane \#11): circular pBR322 
DNA (15.2 $\mu \mathrm{M}$ in base pairs), 5\% DMSO (vehicle), phosphate buffer (10 mM sodium phosphate, $\mathrm{pH} 7.0), 16 \mathrm{~h}, 37^{\circ} \mathrm{C}$. Conditions (Lanes \#12-\#19): circular pBR322 DNA (15.2 $\mu \mathrm{M}$ in base pairs), $4(500 \mu \mathrm{M}-100 \mathrm{nM}), 5 \%$ DMSO, phosphate buffer (10 mM sodium phosphate, $\mathrm{pH} 7.0), 16 \mathrm{~h}, 37$ ${ }^{\circ} \mathrm{C}$. The DNA was analyzed by native gel electrophoresis $(90 \mathrm{~V}, 1.5 \mathrm{~h})$. 


\subsection{DNA crosslinking assay of (-)-104 and (+)-104.}

Linearized pUC19 DNA was used for all DNA cross-linking assays. To prepare the linearized DNA, the 2686 bp pUC19 vector (New England Biolabs ${ }^{\circledR}$ ) was linearized with 20 units/ $\mu \mathrm{g}$ EcoRIHF ${ }^{\circledR}$ (New England Biolabs ${ }^{\circledR}$ ) and the linearized DNA was purified using a PCR clean-up kit (New England Biolabs ${ }^{\circledR}$ ), eluted into $10 \mathrm{mM}$ Tris $(\mathrm{pH} 8.0)$, and quantified using a nanodrop. For each reaction with (-)-104 or (+)-ent-104, $200 \mathrm{ng}$ of linearized pUC19 DNA (15.4 $\mu \mathrm{M}$ base pairs) was incubated with (-)-104 or (+)-ent-104 in concentrations ranging from $100 \mu \mathrm{M}-1 \mu \mathrm{M}$ in a total volume of $20 \mu \mathrm{L}$. (-)-104 or (+)-ent-104 were diluted in DMSO such that each reaction contained a fixed 5\% DMSO concentration. Reactions were conducted in TE buffer $(10 \mathrm{mM}$ Tris and $1 \mathrm{mM}$ EDTA, pH 8.0). Pure cisplatin (Biovision $\left.{ }^{\circledR}\right)$, methyl methanesulfonate (MMS) and DMSO vehicle were used as positive and negative controls, respectively. Stock solutions of cisplatin or MMS in DMSO were prepared immediately before use. To prepare the positive and negative controls, linearized pUC19 DNA (15.4 $\mu \mathrm{M}$ base pairs) was treated with $100 \mu \mathrm{M}$ cisplatin, $500 \mu \mathrm{M}$ MMS, or DMSO vehicle in TE buffer (10 mM Tris and $1 \mathrm{mM}$ EDTA, $\mathrm{pH}$ 8.0) with a final DMSO concentration of $5 \%$. Reactions were conducted for $16 \mathrm{~h}$ at $37^{\circ} \mathrm{C}$. The DNA was stored at $-20^{\circ} \mathrm{C}$ until electrophoretic analysis.

Denaturing gel electrophoresis was applied for DNA cross-linking assays. For each DNA sample, $5 \mu \mathrm{L}(50 \mathrm{ng}$ ) of DNA was removed each time and mixed with $15 \mu \mathrm{L}$ of $0.4 \%$ denaturing buffer ( $0.53 \%$ sodium hydroxide, $10 \%$ glycerol, and $0.013 \%$ bromophenol blue) in an ice bath. The mixed DNA samples were denatured at $4{ }^{\circ} \mathrm{C}$ for $10 \mathrm{~min}$ and then immediately loaded onto $1 \%$ agarose Tris Borate EDTA (TBE) gels. The gel electrophoresis was conducted at $90 \mathrm{~V}$ for $1.5 \mathrm{~h}$ (unless otherwise noted). The gel was stained with SybrGold (Thermo Fisher) for $2 \mathrm{~h}$ before imaging. 


\section{Chiral Separation of the Diosphenol ( \pm )-98.}

Figure S2. Analytical chiral SFC-UV-ESIMS of ( \pm )-98 with Chiralpak® IC column (5 $\mu \mathrm{m}$ particle size, $2.1 \times 150 \mathrm{~mm}$ ).

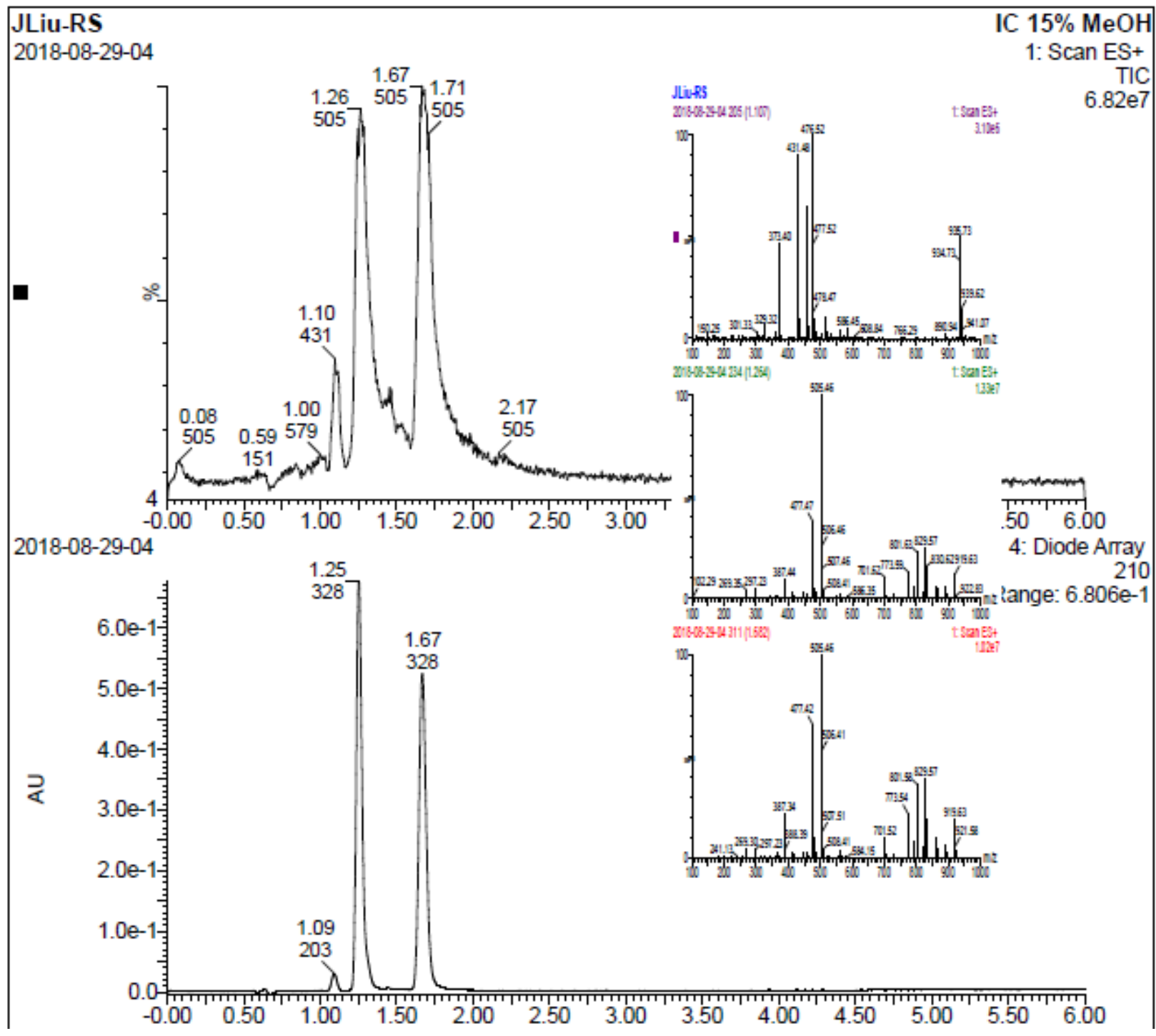


Figure S3. Enantiopurity analysis of separated (-)-98.

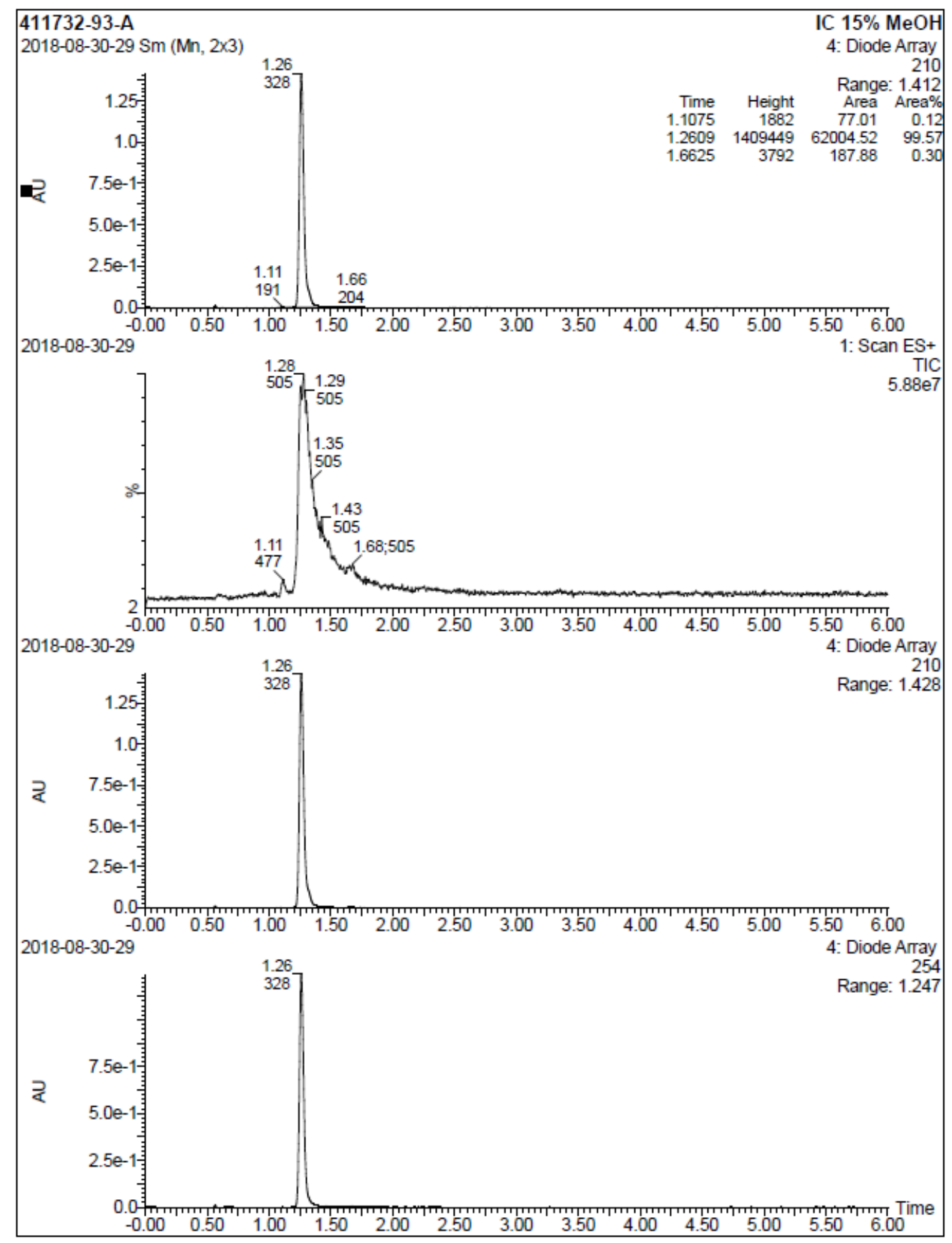


Figure S4. Enantiopurity analysis of separated (+)-98.

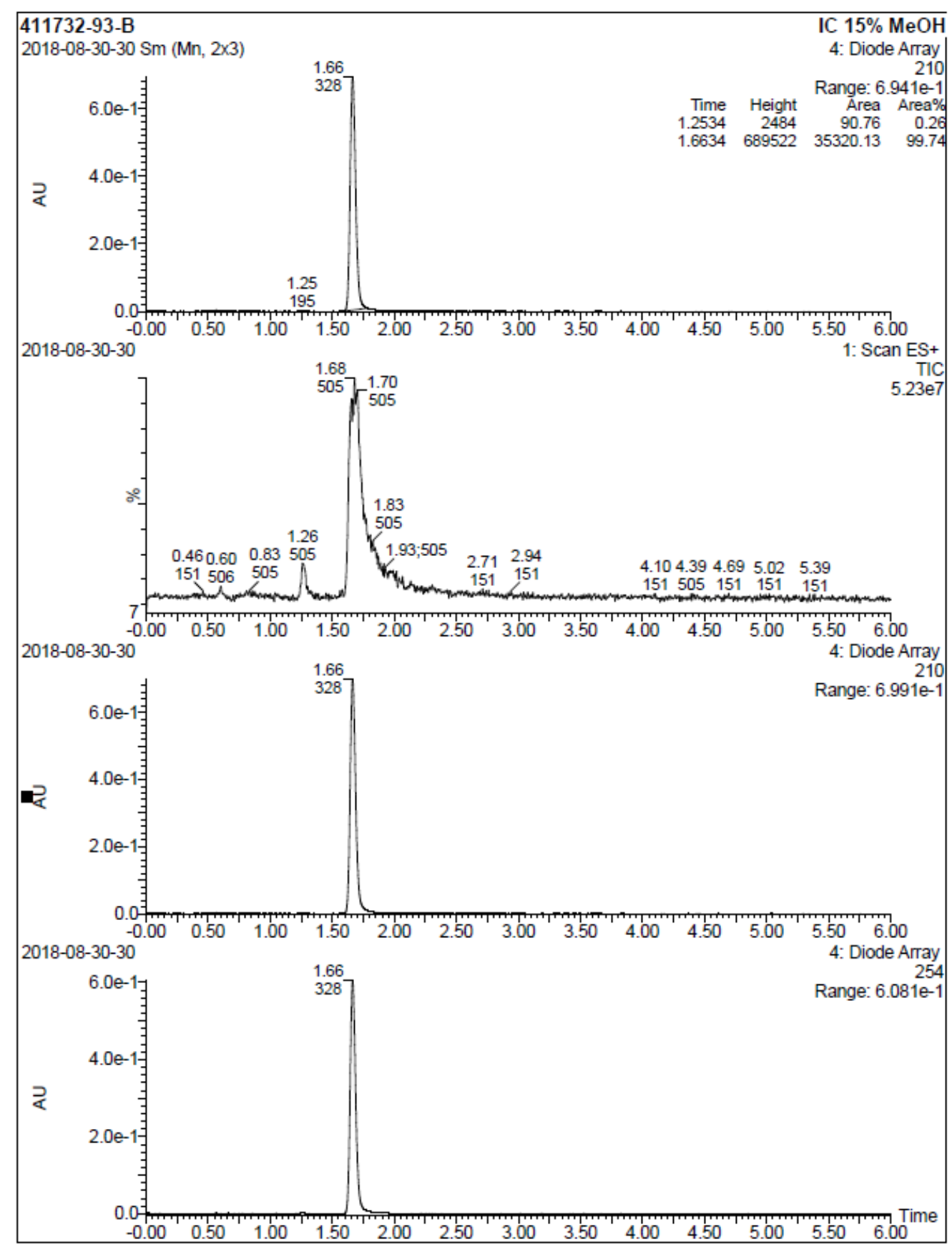




\section{Crystallographic Data for Compound 36.}

Single crystals of compound $\mathbf{3 6}$ suitable for the X-ray crystal structure analysis were obtained by the slow evaporation of a solution of compound 36 in $n$-pentane/diethyl ether $(\mathrm{v} / \mathrm{v}=6: 1)$ at room temperature. Low-temperature diffraction data ( $\omega$-scans) were collected on a Rigaku MicroMax$007 \mathrm{HF}$ diffractometer coupled to a Saturn994+ CCD detector with $\mathrm{Cu} \mathrm{K \alpha}(\lambda=1.54178 \AA)$ for the structure of 007-17027. The diffraction images were processed and scaled using Rigaku Oxford Diffraction software (CrysAlisPro; Rigaku OD: The Woodlands, TX, 2015). The structure was solved with SHELXT and was refined against $\mathrm{F}^{2}$ on all data by full-matrix least squares with SHELXL (Sheldrick, G. M. Acta Cryst. 2008, A64, 112-122). All non-hydrogen atoms were refined anisotropically. Hydrogen atoms were included in the model at geometrically calculated positions and refined using a riding model. The isotropic displacement parameters of all hydrogen atoms were fixed to 1.2 times the $U$ value of the atoms to which they are linked (1.5 times for methyl groups). The alcohol protons $\mathrm{H} 1$ and $\mathrm{H} 9$ were found in the difference map and semi-freely refined with a distance restraint of $0.84(2)$, the expected value for $\mathrm{O}-\mathrm{H}$ at $93 \mathrm{~K}$. Proton $\mathrm{H} 5$ was geometrically placed and refined as a riding atom on $\mathrm{O} 5$. The full numbering scheme of compound 007-17027 can be found in the full details of the X-ray structure determination (CIF), which is included as Supporting Information. CCDC number 1992799 (007-17027) contains the supplementary crystallographic data for this paper. These data can be obtained free of charge from The Cambridge Crystallographic Data Center via www.ccdc.cam.ac.uk/data_request/cif.

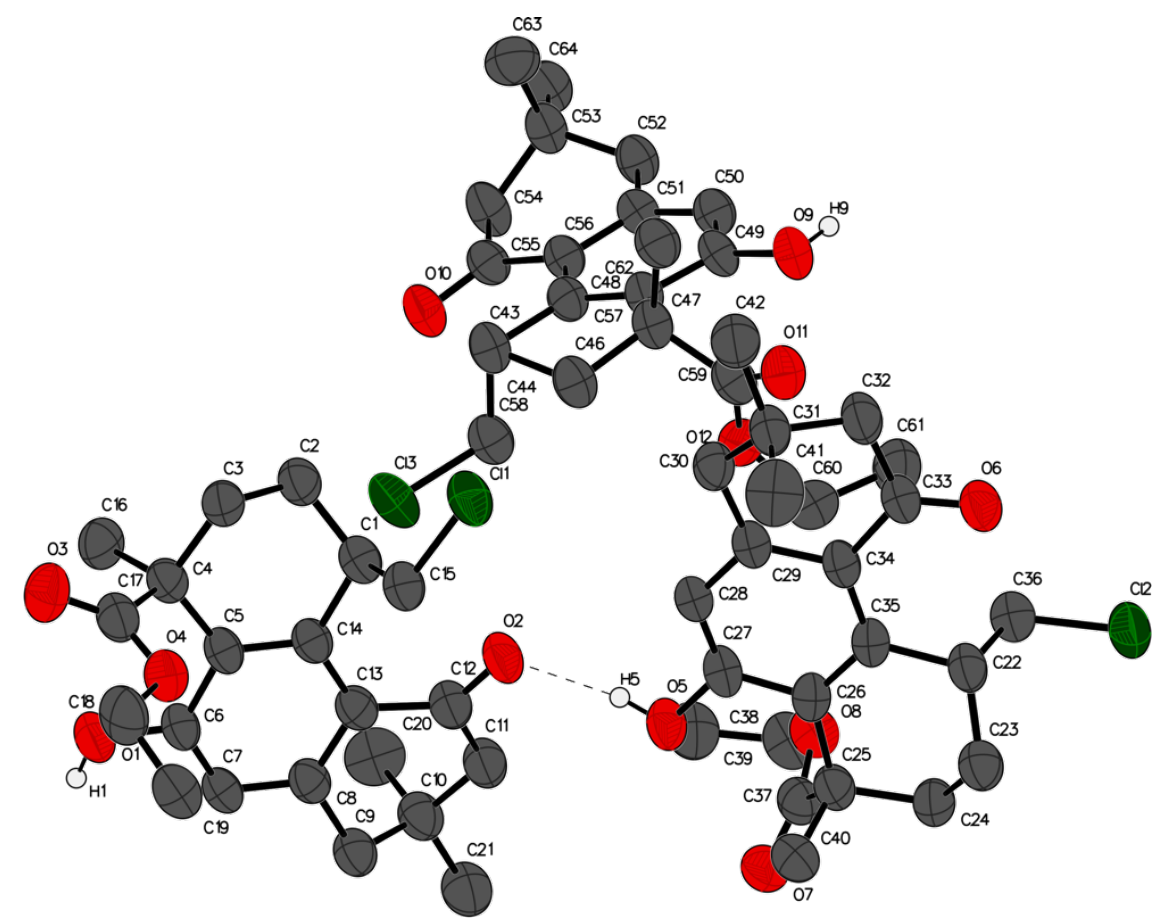

Figure S5. The complete numbering scheme of compound $\mathbf{3 6}$ with 50\% thermal ellipsoid probability levels. The hydrogen atoms are shown as circles for clarity. 
X-ray crystal data and structure refinement for compound $\mathbf{3 6}$.

Identification code

Empirical formula

Formula weight

Temperature

Wavelength

Crystal system

Space group

Unit cell dimensions

Volume

Z

Density (calculated)

Absorption coefficient

$\mathrm{F}(000)$

Crystal size

Theta range for data collection

Index ranges

Reflections collected

Independent reflections

Completeness to theta $=67.684^{\circ}$

Absorption correction

Max. and min. transmission

Refinement method

Data / restraints / parameters

Goodness-of-fit on $\mathrm{F}^{2}$

Final R indices [I $>2 \operatorname{sigma(I)]~}$

$\mathrm{R}$ indices (all data)

Absolute structure parameter

Largest diff. peak and hole
007-17027

$\mathrm{C} 21 \mathrm{H} 27 \mathrm{Cl} \mathrm{O} 4$

378.87

93(2) K

\section{$1.54184 \AA$}

Orthorhombic

Fdd 2

$\mathrm{a}=64.915(3) \AA \quad \alpha=90^{\circ}$.

$\mathrm{b}=22.4355(12) \AA \quad \beta=90^{\circ}$.

$\mathrm{c}=16.2049(8) \AA \quad \gamma=90^{\circ}$.

23601(2) $\AA^{3}$

48

$1.280 \mathrm{Mg} / \mathrm{m}^{3}$

$1.904 \mathrm{~mm}^{-1}$

9696

$0.200 \times 0.010 \times 0.010 \mathrm{~mm}^{3}$

2.723 to $68.383^{\circ}$.

$-78<=\mathrm{h}<=78,-27<=\mathrm{k}<=26,-19<=\mathrm{k}<=19$

216426

$10802[\mathrm{R}$ (int) $=0.1978]$

$100.0 \%$

Semi-empirical from equivalents

1.00000 and 0.45037

Full-matrix least-squares on $\mathrm{F}^{2}$

$10802 / 3 / 724$

1.041

$\mathrm{R} 1=0.0641, \mathrm{wR} 2=0.1602$

$\mathrm{R} 1=0.0952, \mathrm{wR} 2=0.1814$

$-0.005(11)$

0.375 and -0.304 e. $\AA^{-}-3$ 


\section{Crystallographic Data for Compound 65.}

Single crystals of compound $\mathbf{6 5}$ suitable for the X-ray crystal structure analysis were obtained by the slow evaporation of a solution of compound $\mathbf{6 5}$ in $n$-pentane/diethyl ether $(\mathrm{v} / \mathrm{v}=10: 1)$ at room temperature. Low-temperature diffraction data ( $\omega$-scans) were collected on a Rigaku MicroMax$007 \mathrm{HF}$ diffractometer coupled to a Saturn994+ $\mathrm{CCD}$ detector with $\mathrm{Cu} \mathrm{K} \alpha(\lambda=1.54178 \AA)$ for the structure of 007-15166. The diffraction images were processed and scaled using the Rigaku CrystalClear software (CrystalClear and CrystalStructure; Rigaku/MSC: The Woodlands, TX, 2005). The structure was solved with SHELXT and was refined against $F^{2}$ on all data by fullmatrix least squares with SHELXL (Sheldrick, G. M. Acta Cryst. 2008, A64, 112-122). All nonhydrogen atoms were refined anisotropically. Hydrogen atoms were included in the model at geometrically calculated positions and refined using a riding model. The isotropic displacement parameters of all hydrogen atoms were fixed to 1.2 times the $U$ value of the atoms to which they are linked (1.5 times for methyl groups). The full numbering scheme of compound 007-15166 can be found in the Supporting Information. Full details of the X-ray structure determination are in the CIF included as Supporting Information. CCDC number 1992798 (007-15166) contains the supplementary crystallographic data for this paper. These data can be obtained free of charge from The Cambridge Crystallographic Data Center via www.ccdc.cam.ac.uk/data request/cif.

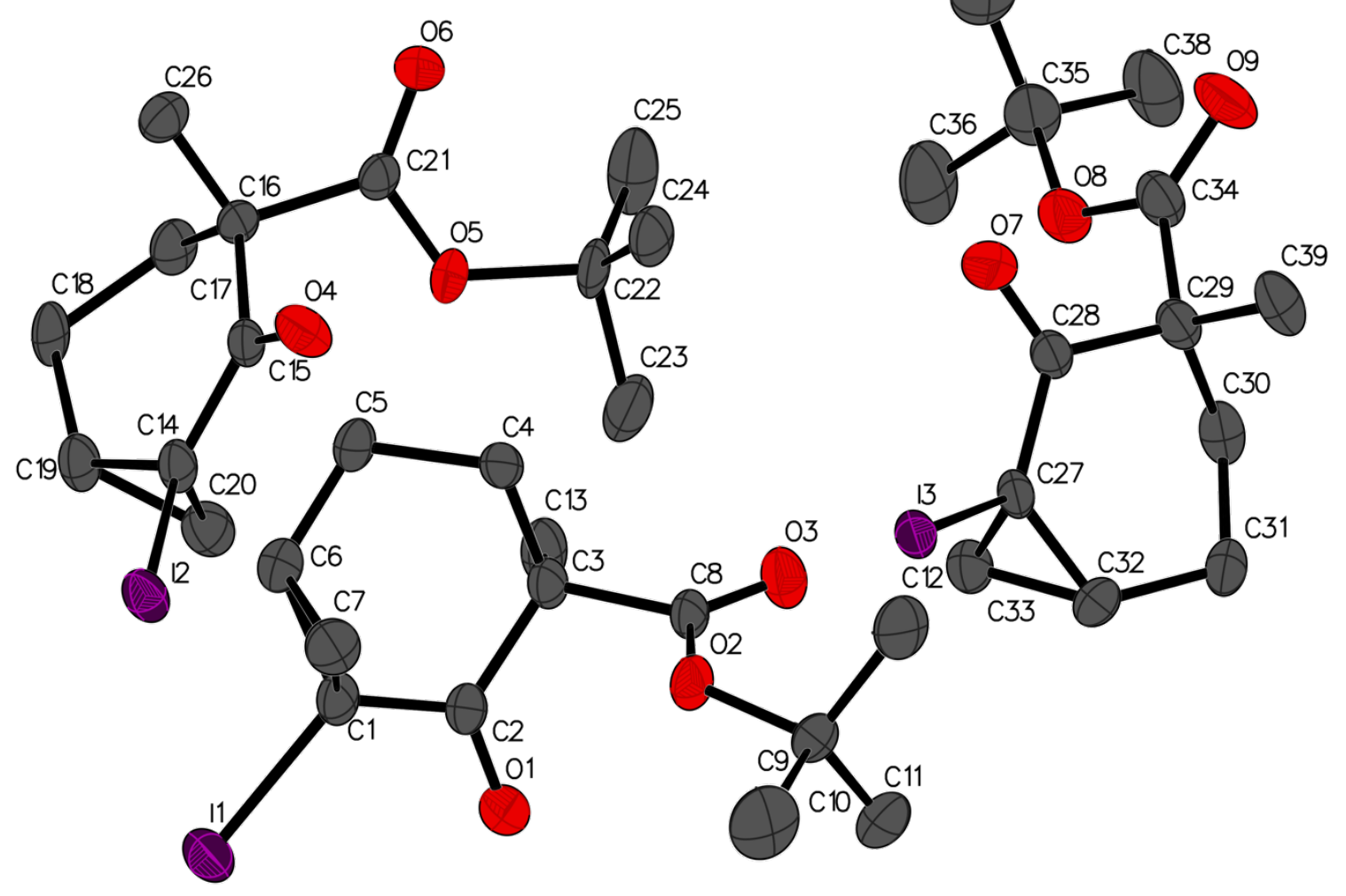

Figure S6. The full numbering scheme of compound $\mathbf{6 5}$ with $50 \%$ thermal ellipsoid probability levels. The hydrogen atoms have been omitted for clarity. 
X-ray crystal data and structure refinement for compound 65 .

Identification code

Empirical formula

Formula weight

Temperature

Wavelength

Crystal system

Space group

Unit cell dimensions

Volume

Z

Density (calculated)

Absorption coefficient

$\mathrm{F}(000)$

Crystal size

Crystal color and habit

Diffractometer

Theta range for data collection

Index ranges

Reflections collected

Independent reflections

Observed reflections (I $>2$ sigma(I))

Completeness to theta $=66.592^{\circ}$

Absorption correction

Max. and min. transmission

Solution method

Refinement method

Data / restraints / parameters

Goodness-of-fit on $\mathrm{F}^{2}$

Final R indices [I $>2 \operatorname{sigma}(\mathrm{I})]$

$\mathrm{R}$ indices (all data)

Largest diff. peak and hole
007-15166

$\mathrm{C}_{13} \mathrm{H}_{19} \mathrm{IO}_{3}$

350.18

93(2) K

\section{$1.54178 \AA$}

Monoclinic

$\mathrm{P} 21 / \mathrm{n}$

$\mathrm{a}=24.9006(17) \AA \quad \alpha=90^{\circ}$.

$\mathrm{b}=6.3095(4) \AA$

$\beta=110.855(2)^{\circ}$.

$\mathrm{c}=28.910(2) \AA$

$\gamma=90^{\circ}$.
12

$1.644 \mathrm{Mg} / \mathrm{m}^{3}$

$17.753 \mathrm{~mm}^{-1}$

2088

$0.200 \times 0.080 \times 0.020 \mathrm{~mm}^{3}$

Colorless Needel

Rigaku Saturn 944+ CCD

3.272 to $66.592^{\circ}$.

$-29<=\mathrm{h}<=28,-7<=\mathrm{k}<=7,-34<=1<=34$

131210

$7415[\mathrm{R}(\mathrm{int})=0.1267]$

6646

$98.5 \%$

Semi-empirical from equivalents

0.718 and 0.384

SHELXT-2014/5 (Sheldrick, 2014)

SHELXL-2014/7 (Sheldrick, 2014)

7415 / 0 / 472

1.019

$\mathrm{R} 1=0.0474, \mathrm{wR} 2=0.1220$

$\mathrm{R} 1=0.0519, \mathrm{wR} 2=0.1262$

1.079 and -1.984 e. $\AA^{-3}$ 


\section{Crystallographic Data for Compound 69.}

Single crystals of compound 69 suitable for the X-ray crystal structure analysis were obtained by the slow evaporation of a solution of compound 69 in $n$-pentane/diethyl ether $(\mathrm{v} / \mathrm{v}=20: 1)$ at room temperature. Low-temperature diffraction data ( $\omega$-scans) were collected on a Rigaku MicroMax$007 \mathrm{HF}$ diffractometer coupled to a Saturn994+ $\mathrm{CCD}$ detector with $\mathrm{Cu} \mathrm{K} \alpha(\lambda=1.54178 \AA)$ for the structure of 007-17082. The diffraction images were processed and scaled using Rigaku Oxford Diffraction software (CrysAlisPro; Rigaku OD: The Woodlands, TX, 2015). The structure was solved with SHELXT and was refined against $\mathrm{F}^{2}$ on all data by full-matrix least squares with SHELXL (Sheldrick, G. M. Acta Cryst. 2008, A64, 112-122). All non-hydrogen atoms were refined anisotropically. Hydrogen atoms were included in the model at geometrically calculated positions and refined using a riding model. The isotropic displacement parameters of all hydrogen atoms were fixed to 1.2 times the $U$ value of the atoms to which they are linked (1.5 times for methyl groups). The only exceptions are $\mathrm{H} 1$ and $\mathrm{H} 5$, which were found and semi-freely refined with distance restraints of 0.83(2), as suggested by the difference map.The full numbering scheme of compound 007-17082 can be found in the full details of the X-ray structure determination (CIF), which is included as Supporting Information. CCDC number 1992803 (007-17082) contains the supplementary crystallographic data for this paper. These data can be obtained free of charge from The Cambridge Crystallographic Data Center via www.ccdc.cam.ac.uk/data request/cif.

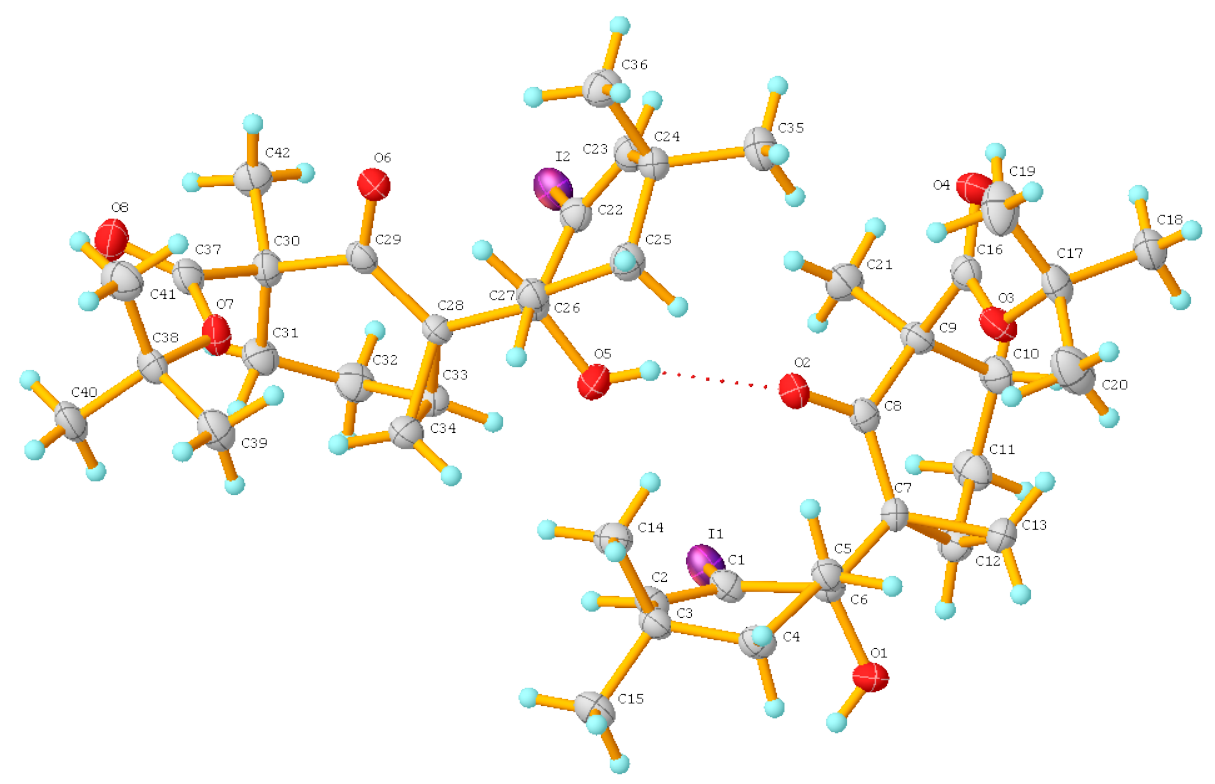

Figure S7. The complete numbering scheme of compound 69 with 50\% thermal ellipsoid probability levels. The hydrogen atoms are shown as circles for clarity. Dashed lines highlight hydrogen bond interactions. 
$\mathrm{X}$-ray crystal data and structure refinement for compound 69.

Identification code

Empirical formula

Formula weight

Temperature

Wavelength

Crystal system

Space group

Unit cell dimensions

Volume

Z

Density (calculated)

Absorption coefficient

$\mathrm{F}(000)$

Crystal size

Crystal color and habit

Diffractometer

Theta range for data collection

Index ranges

Reflections collected

Independent reflections

Observed reflections (I > 2sigma(I))

Completeness to theta $=66.598^{\circ}$

Absorption correction

Max. and min. transmission

Solution method

Refinement method

Data / restraints / parameters

Goodness-of-fit on $\mathrm{F}^{2}$

Final $\mathrm{R}$ indices [I $>2 \operatorname{sigma}(\mathrm{I})]$

$\mathrm{R}$ indices (all data)

Largest diff. peak and hole
007-17082

C21 H31 I O4

474.36

93(2) K

\section{$1.54184 \AA$}

Monoclinic

$\mathrm{P} 21 / \mathrm{c}$

$\mathrm{a}=17.2644(4) \AA \quad \alpha=90^{\circ}$.

$\mathrm{b}=11.1326(3) \AA$

$\beta=110.142(2)^{\circ}$.

$\mathrm{c}=23.6238(5) \AA$

$\gamma=90^{\circ}$.

4262.75(18) $\AA^{3}$

8

$1.478 \mathrm{Mg} / \mathrm{m}^{3}$

$11.978 \mathrm{~mm}^{-1}$

1936

$0.100 \times 0.100 \times 0.020 \mathrm{~mm}^{3}$

Colorless Plate

Rigaku Saturn 944+ CCD

2.726 to $66.598^{\circ}$. $-20<=\mathrm{h}<=20,-13<=\mathrm{k}<=12,-28<=\mathrm{l}<=28$

151643

$7514[\mathrm{R}(\mathrm{int})=0.0881]$

6222

$99.8 \%$

Semi-empirical from equivalents

1.00000 and 0.59010

SHELXT-2014/5 (Sheldrick, 2014)

SHELXL-2014/7 (Sheldrick, 2014)

7514 / 2 / 489

1.057

$\mathrm{R} 1=0.0381, \mathrm{wR} 2=0.0832$

$\mathrm{R} 1=0.0514, \mathrm{wR} 2=0.0892$

0.831 and -1.078 e. $\AA^{-3}$ 


\section{Crystallographic Data for Compound 74.}

Single crystals of compound $\mathbf{7 4}$ suitable for the X-ray crystal structure analysis were obtained by the slow evaporation of a solution of compound $\mathbf{7 4}$ in $n$-hexanes/diethyl ether $(\mathrm{v} / \mathrm{v}=4: 1)$ at room temperature. Low-temperature diffraction data ( $\omega$-scans) were collected on a Rigaku MicroMax$007 \mathrm{HF}$ diffractometer coupled to a Saturn994+ $\mathrm{CCD}$ detector with $\mathrm{Cu} \mathrm{K} \alpha(\lambda=1.54178 \AA)$ for the structure of 007a-18078. The diffraction images were processed and scaled using Rigaku Oxford Diffraction software (CrysAlisPro; Rigaku OD: The Woodlands, TX, 2015). The structure was solved with SHELXT and was refined against $\mathrm{F}^{2}$ on all data by full-matrix least squares with SHELXL (Sheldrick, G. M. Acta Cryst. 2008, A64, 112-122). All non-hydrogen atoms were refined anisotropically. Hydrogen atoms were included in the model at geometrically calculated positions and refined using a riding model. The isotropic displacement parameters of all hydrogen atoms were fixed to 1.2 times the $\mathrm{U}$ value of the atoms to which they are linked (1.5 times for methyl groups). The only exception are the hydrogen atoms associated with oxygen atoms. These sites were found in the difference map and freely refined (see Table 7). The full numbering scheme of compound $007 \mathrm{a}-18078$ can be found in the full details of the X-ray structure determination (CIF), which is included as Supporting Information. CCDC number 1992800 (007a-18078) contains the supplementary crystallographic data for this paper. These data can be obtained free of charge from The Cambridge Crystallographic Data Center via www.ccdc.cam.ac.uk/data_request/cif.

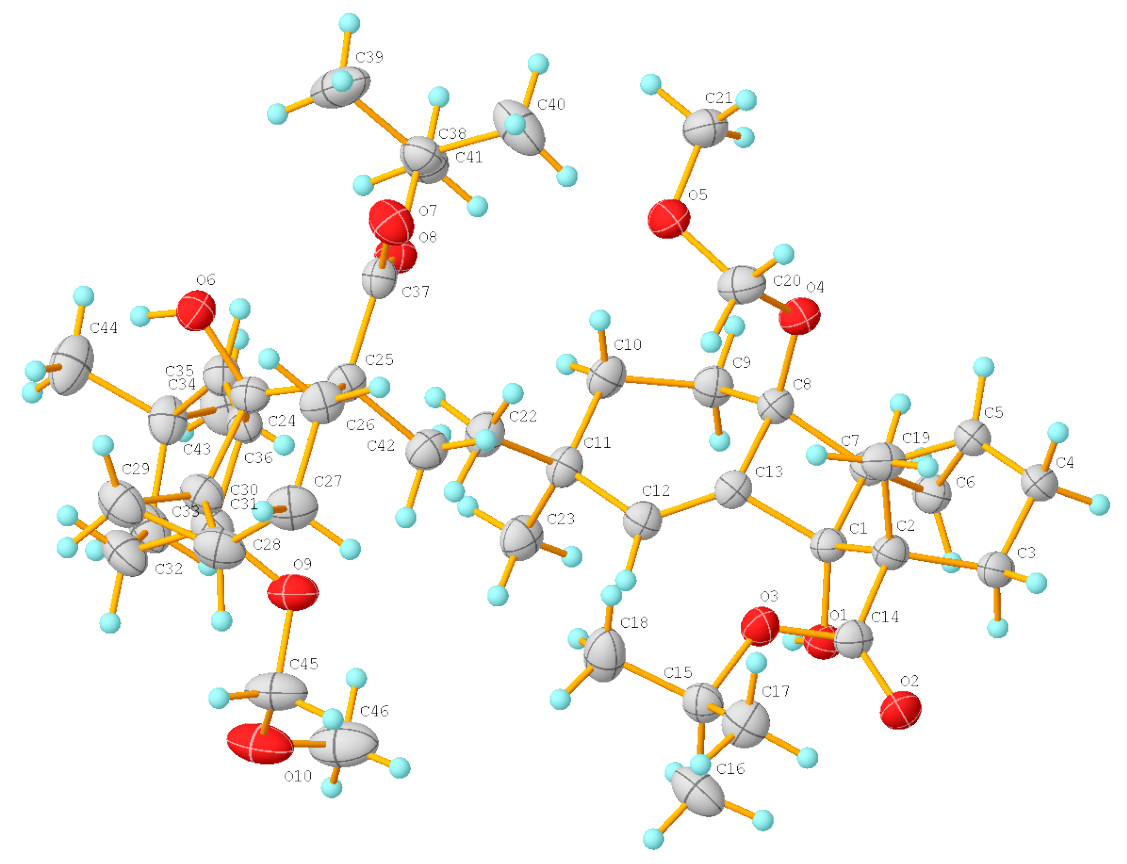

Figure S8. The complete numbering scheme of compound $\mathbf{7 4}$ with 50\% thermal ellipsoid probability levels. The hydrogen atoms are shown as circles for clarity. 
X-ray crystal data and structure refinement for compound $\mathbf{7 4}$.

Identification code

Empirical formula

Formula weight

Temperature

Wavelength

Crystal system

Space group

Unit cell dimensions

Volume

Z

Density (calculated)

Absorption coefficient

$\mathrm{F}(000)$

Crystal size

Crystal color and habit

Diffractometer

Theta range for data collection

Index ranges

Reflections collected

Independent reflections

Observed reflections (I > 2sigma(I))

Completeness to theta $=66.599^{\circ}$

Absorption correction

Max. and min. transmission

Solution method

Refinement method

Data / restraints / parameters

Goodness-of-fit on $\mathrm{F}^{2}$

Final R indices [I $>2 \operatorname{sigma}(\mathrm{I})]$

$\mathrm{R}$ indices (all data)

Absolute structure parameter

Largest diff. peak and hole 007a-18078

C23 H36 O5

392.52

93(2) K

\section{$1.54184 \AA$}

Orthorhombic

Pca21

$\mathrm{a}=19.7853(3) \AA \quad \alpha=90^{\circ}$.

$\mathrm{b}=22.1644(4) \AA \quad \beta=90^{\circ}$.

$\mathrm{c}=10.4000(2) \AA \quad \gamma=90^{\circ}$.

$4560.70(14) \AA^{3}$

8

$1.143 \mathrm{Mg} / \mathrm{m}^{3}$

$0.633 \mathrm{~mm}^{-1}$

1712

$0.100 \times 0.100 \times 0.070 \mathrm{~mm}^{3}$

Colorless Block

Rigaku Saturn 944+ CCD

1.993 to $66.599^{\circ}$.

$-23<=\mathrm{h}<=23,-26<=\mathrm{k}<=26,-12<=1<=12$

164334

8074 [R(int) $=0.0727]$

7400

$100.0 \%$

Semi-empirical from equivalents

1.00000 and 0.65736

SHELXT-2014/5 (Sheldrick, 2014)

SHELXL-2014/7 (Sheldrick, 2014)

8074 / 1 / 527

1.035

$\mathrm{R} 1=0.0318, \mathrm{wR} 2=0.0736$

$\mathrm{R} 1=0.0368, \mathrm{wR} 2=0.0761$

$0.07(6)$

0.218 and -0.120 e. $\AA^{-}-3$ 


\section{Crystallographic Data for Compound 89.}

Single crystals of compound $\mathbf{8 9}$ suitable for the X-ray crystal structure analysis were obtained by the slow evaporation of a solution of compound 89 in $n$-pentane/diethyl ether $(\mathrm{v} / \mathrm{v}=8: 1)$ at room temperature. Low-temperature diffraction data ( $\omega$-scans) were collected on a Rigaku MicroMax$007 \mathrm{HF}$ diffractometer coupled to a Dectris Pilatus3R detector with Mo K $\alpha(\lambda=0.71073 \AA)$ for the structure of 007c-17050. The diffraction images were processed and scaled using Rigaku Oxford Diffraction software (CrysAlisPro; Rigaku OD: The Woodlands, TX, 2015). The structure was solved with SHELXT and was refined against $\mathrm{F}^{2}$ on all data by full-matrix least squares with SHELXL (Sheldrick, G. M. Acta Cryst. 2008, A64, 112-122). All non-hydrogen atoms were refined anisotropically. Hydrogen atoms were included in the model at geometrically calculated positions and refined using a riding model. The isotropic displacement parameters of all hydrogen atoms were fixed to 1.2 times the $U$ value of the atoms to which they are linked (1.5 times for methyl groups). Several low angle reflections were recorded improperly and omitted from the refinement. The full numbering scheme of compound $007 \mathrm{c}-17050$ can be found in the full details of the X-ray structure determination (CIF), which is included as Supporting Information. CCDC number 1992802 (007c-17050) contains the supplementary crystallographic data for this paper. These data can be obtained free of charge from The Cambridge Crystallographic Data Center via www.ccdc.cam.ac.uk/data_request/cif.

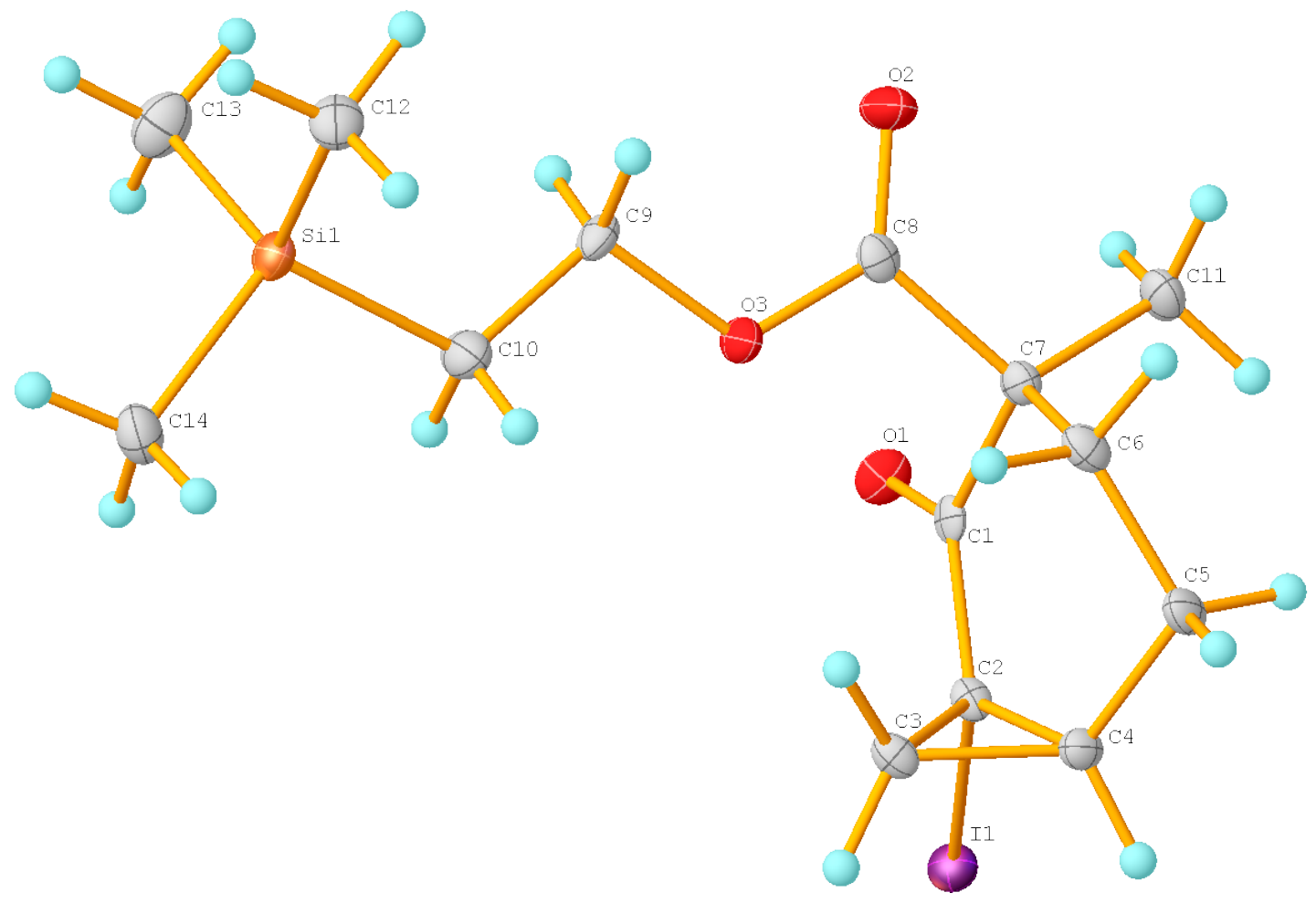

Figure S9. The complete numbering scheme of compound 89 with $50 \%$ thermal ellipsoid probability levels. The hydrogen atoms are shown as circles for clarity. 
X-ray Crystal data and structure refinement for compound $\mathbf{8 9}$.

Identification code

Empirical formula

Formula weight

Temperature

Wavelength

Crystal system

Space group

Unit cell dimensions

Volume

Z

Density (calculated)

Absorption coefficient

$\mathrm{F}(000)$

Crystal size

Crystal color and habit

Diffractometer

Theta range for data collection

Index ranges

Reflections collected

Independent reflections

Observed reflections (I $>2 \operatorname{sigma}(\mathrm{I})$ )

Completeness to theta $=25.242^{\circ}$

Absorption correction

Max. and min. transmission

Solution method

Refinement method

Data / restraints / parameters

Goodness-of-fit on $\mathrm{F}^{2}$

Final $\mathrm{R}$ indices [I $>2 \operatorname{sigma}(\mathrm{I})]$

$\mathrm{R}$ indices (all data)

Largest diff. peak and hole 007c-17050

C14 H23 I O3 Si

394.31

93(2) K

$0.71073 \AA$

Monoclinic

$\mathrm{P} 21 / \mathrm{c}$

$\mathrm{a}=21.9715(15) \AA \quad \alpha=90^{\circ}$.

$\mathrm{b}=6.3739(4) \AA$

$\beta=92.562(6)^{\circ}$.

$\mathrm{c}=12.0779(7) \AA$

$1689.75(18) \AA^{3}$

4

$1.550 \mathrm{Mg} / \mathrm{m}^{3}$

$1.968 \mathrm{~mm}^{-1}$

792

$0.200 \times 0.150 \times 0.010 \mathrm{~mm}^{3}$

Colorless Plate

Dectris Pilatus 3R

3.328 to $27.483^{\circ}$.

$-28<=\mathrm{h}<=28,-8<=\mathrm{k}<=6,-15<=\mathrm{k}<=15$

19769

$3860[\mathrm{R}(\mathrm{int})=0.0502]$

3463

$99.7 \%$

Semi-empirical from equivalents

1.00000 and 0.30279

SHELXT-2014/5 (Sheldrick, 2014)

SHELXL-2014/7 (Sheldrick, 2014)

3860 / 0 / 176

1.077

$\mathrm{R} 1=0.0356, \mathrm{wR} 2=0.0865$

$\mathrm{R} 1=0.0402, \mathrm{wR} 2=0.0883$

1.647 and -1.213 e. $\AA^{-3}$ 


\section{Crystallographic Data for Compound (-)-104.}

Crystals of compound (-)-104 for the X-ray crystal structure analysis were obtained by the slow evaporation of a solution of compound $(-)-104$ in ethyl acetate/methanol $(\mathrm{v} / \mathrm{v}=5: 1)$ at room temperature. Low-temperature diffraction data ( $\omega$-scans) were collected on a Rigaku MicroMax$007 \mathrm{HF}$ diffractometer coupled to a Saturn994+ $\mathrm{CCD}$ detector with $\mathrm{Cu} \mathrm{K} \alpha(\lambda=1.54178 \AA)$ for the structure of 007b-20019. The diffraction images were processed and scaled using Rigaku Oxford Diffraction software (CrysAlisPro; Rigaku OD: The Woodlands, TX, 2015). The structure was solved with SHELXT and was refined against $\mathrm{F}^{2}$ on all data by full-matrix least squares with SHELXL (Sheldrick, G. M. Acta Cryst. 2008, A64, 112-122). All non-hydrogen atoms were refined anisotropically. Hydrogen atoms were included in the model at geometrically calculated positions and refined using a riding model. The isotropic displacement parameters of all hydrogen atoms were fixed to 1.2 times the $\mathrm{U}$ value of the atoms to which they are linked (1.5 times for methyl groups). The only exceptions are $\mathrm{H} 1, \mathrm{H} 3$, and $\mathrm{H} 5$ which were found in the difference map and freely refined. The full numbering scheme of compound 007b-20019 can be found in the full details of the X-ray structure determination (CIF), which is included as Supporting Information. CCDC number 1992801 (007b-20019) contains the supplementary crystallographic data for this paper. These data can be obtained free of charge from The Cambridge Crystallographic Data Center via www.ccdc.cam.ac.uk/data request/cif.

The crystal used for the diffraction experiment was small and only had light atoms present which made measuring any differences from anomalous dispersion in Bijvoet pairs difficult. The output from the analysis performed by SHELXL is copied below.

Flack $\mathrm{x}=-0.476(533)$ by classical fit to all intensities -0.876 (401) from 791 selected quotients (Parsons' method)

However, A Bayesian statistical analysis of the Bijvoet pairs suggest the model reported here, ()-104 with stereocenters $S, S, S, S$, is the most likely model, with only an exceedingly small probability of a model with the opposite chirality or a racemic twin. This analysis was calculated with the PLATON software package (A.L.Spek, Acta Cryst. 2009, D65, 148-155.).

\section{$\underline{S, S, S, S \text { model }}$}

\begin{tabular}{lc} 
Space Group & $P 2_{1}$ \\
Wavelength & 1.54184 \\
Flack x ... & \multicolumn{2}{c}{$-0.9(4)$} \\
Parsons z .. & $-0.5(2)$
\end{tabular}

$\begin{array}{ll}\text { Bijvoet Pairs } & 1310 \\ \text { Coverage ... } & 98 \\ \text { DiffCalcMax. } & 9.24 \\ \text { Outlier Crit } & 18.48\end{array}$


Student-T Prob. Plot

Sample Size.

1300

Corr. Coeff. 0.999

Intercept .. 0.291

Slope ...... 1.222

Bayesian Statistics

Student_T Nu

39

Select Pairs 1310

Theta Min .. $\quad 7.94$

Theta_Max .. $\quad 66.55$

P2(true).... 1.000

P3(true).... 0.990

P3(racemic-twin) $\quad 0.010$

P3(false) .. $\quad 0.6 \mathrm{E}-05$

$\mathrm{G}$.......... $\quad 2.1385$

$\mathrm{G}(\mathrm{su}) \ldots . . \quad 0.5973$

Hooft y .... - $\quad-0.6(3)$

\section{$\underline{R, R, R, R \text { model }}$}

Space Group $\quad P 21$

Wavelength 1.54184

Flack x .... 1.9(4)

Parsons z .. $1.5(2)$

Bijvoet Pairs $\quad 1310$

Coverage ... 98

DiffCalcMax. $\quad 9.25$

Outlier Crit $\quad 18.50$

Student-T Prob. Plot

Sample Size.

1300

Corr. Coeff. 0.999

Intercept .. 0.291

Slope ...... 1.222

Bayesian Statistics

Student_T Nu

Select Pairs 1310

Theta_Min .. $\quad 7.94$

Theta_Max .. $\quad 66.55$ 

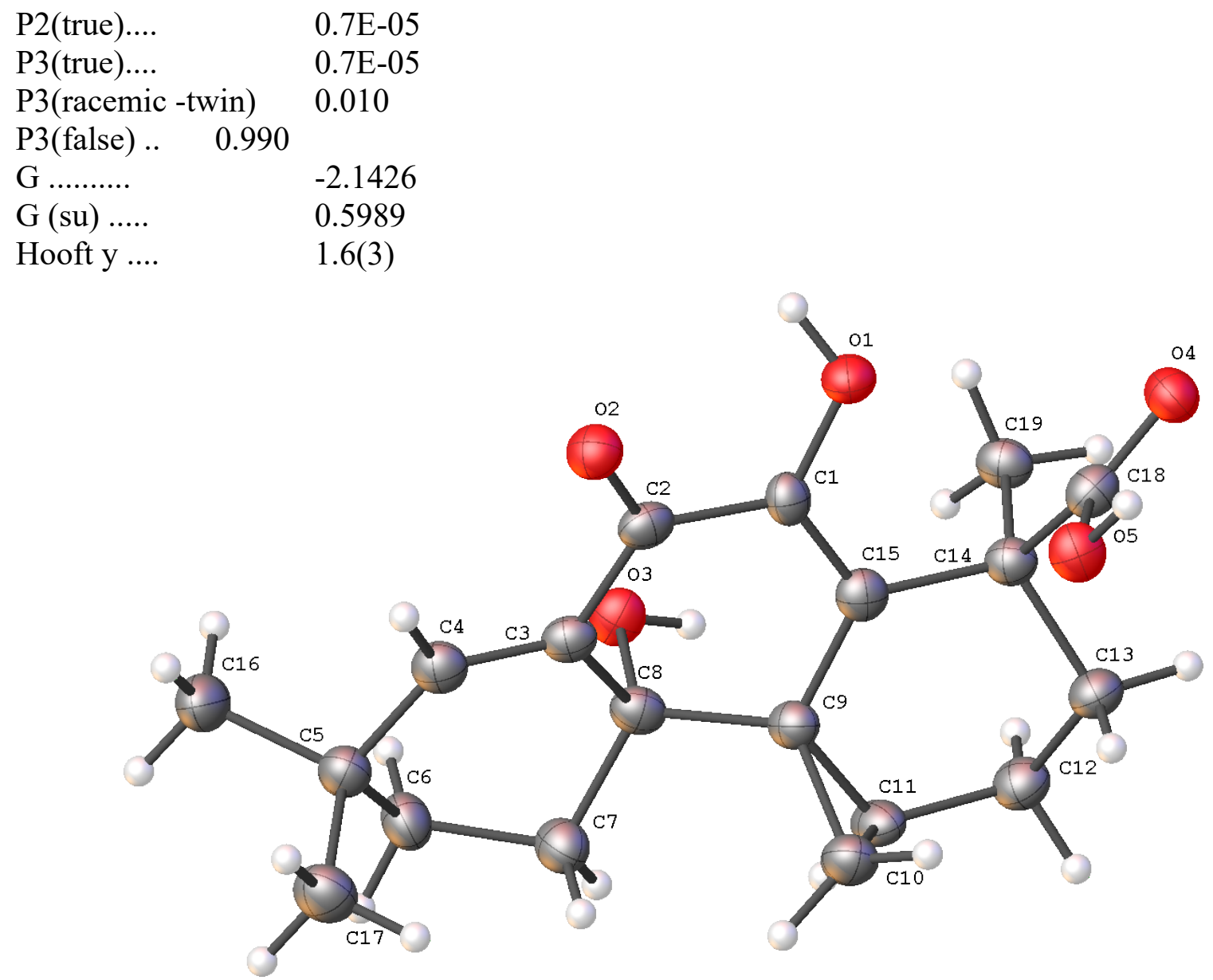

Figure S10. The complete numbering scheme of compound (-)-104 with 50\% thermal ellipsoid probability levels. The hydrogen atoms are shown as circles for clarity.

X-ray crystal data and structure refinement for compound (-)-104.

Identification code

Empirical formula

Formula weight

Temperature

Wavelength

Crystal system

Space group

Unit cell dimensions 007b-20019

C19 H24 O5

332.38

93(2) K

\section{$1.54184 \AA$}

Monoclinic

P21

$$
\begin{array}{ll}
\mathrm{a}=11.2611(6) \AA & \alpha=90^{\circ} . \\
\mathrm{b}=6.3708(2) \AA & \beta=105.942(5)^{\circ} .
\end{array}
$$


Volume

Z

Density (calculated)

Absorption coefficient

$\mathrm{F}(000)$

Crystal size

Crystal color and habit

Diffractometer

Theta range for data collection

Index ranges

Reflections collected

Independent reflections

Observed reflections (I $>2 \operatorname{sigma}(\mathrm{I})$ )

Completeness to theta $=66.548^{\circ}$

Absorption correction

Max. and min. transmission

Solution method

Refinement method

Data / restraints / parameters

Goodness-of-fit on $\mathrm{F}^{2}$

Final $\mathrm{R}$ indices [I $>2 \operatorname{sigma}(\mathrm{I})]$

$\mathrm{R}$ indices (all data)

Absolute structure parameter

Largest diff. peak and hole $\mathrm{c}=12.0672(4) \AA \quad \gamma=90^{\circ}$.

832.43(6) $\AA^{3}$

2

$1.326 \mathrm{Mg} / \mathrm{m}^{3}$

$0.779 \mathrm{~mm}^{-1}$

356

$0.050 \times 0.040 \times 0.010 \mathrm{~mm}^{3}$

Colorless Needle

Rigaku Saturn 944+ CCD

3.810 to $66.548^{\circ}$.

$-13<=\mathrm{h}<=13,-7<=\mathrm{k}<=7,-14<=\mathrm{l}<=14$

28622

$2906[\mathrm{R}(\mathrm{int})=0.1509]$

2243

$98.3 \%$

Semi-empirical from equivalents

1.00000 and 0.32765

SHELXT-2014/5 (Sheldrick, 2014)

SHELXL-2014/7 (Sheldrick, 2014)

$2906 / 1 / 232$

1.030

$\mathrm{R} 1=0.0575, \mathrm{wR} 2=0.1260$

$\mathrm{R} 1=0.0824, \mathrm{wR} 2=0.1414$

$-0.9(4)$

0.168 and -0.216 e. $\AA^{-3}$ 